\title{
Low Noise CMOS Voltage-Control Oscillator Design Methodology with Emphasis on Non-linear Effect Contributions, 2.4 GHz CMOS Design Example
}

by

Zhe Jiang

A thesis submitted to the Faculty of Graduate and Postdoctoral Affairs in partial fulfillment of the requirements for the degree of

Master of Applied Science

in

Electrical and Computer

Carleton University

Ottawa, Ontario

(C) 2014, Zhe Jiang 
The information used in this thesis comes in part from the research program of Dr. Tadeusz Kwasniewski and his associates in the VLSI in Communication group. The research results appearing in this thesis represent an integral part of the ongoing research program. All research results in this thesis including tables, graphs and figures but excluding the narrative portions of the thesis are effectively incorporated into the research program and can be used by Dr. Kwasniewski and his associates for educational and research purpose, including publication in open literature with appropriate credits. The matters intellectual property may be pursed cooperatively with Carleton University and Dr. Kwasniewski and where and when appropriate. 


\begin{abstract}
The Voltage-controlled oscillator (VCO) is one of the most important building blocks in modern communication applications. Although results of many studies have been reported, designing a VCO with low phase noise remains an active research area. Much effort has been devoted to study the generation of phase noise, in order to guide the design and optimization of VCO. However, most of the existing phase noise magnitude expressions are based on linear models. At the same time, practical implementations show attempts to design oscillators operating with fewer harmonics [1].

This thesis demonstrates an attempt to link non-linear (transient) oscillator behavior with phase noise. Phase noise generation is first studied, starting with examining Groszkowski's equation. After studying the mechanism, it is found that phase noise is determined by the transfer function and harmonics and based on which, a VCO design guideline is proposed to aid low phase noise VCO design. Then a novel VCO topology is proposed based on this guideline, aiming to reduce the harmonics and decrease the magnitude and the bandwidth of the transfer function. The circuit was designed and implemented in $0.13 \mathrm{um}$ IBM CMOS technology. The simulated performance at $2.4 \mathrm{GHz}$ was $-133 \mathrm{dBc} / \mathrm{Hz}$ at $1 \mathrm{MHz}$ offset with supply current of $3.07 \mathrm{~mA}$ at $1 \mathrm{~V}$ supply.
\end{abstract}




\section{Acknowledgements}

I would like to express my sincere gratitude to my supervisor, Dr. Kwasniewski, for giving me the opportunity to conduct this research, for his valuable advice and support on this work and thesis, for his concern in all aspects of my study in Carleton University.

I would like to thank Dr. Guo for the discussion throughout my graduate study. Thank Mr. Nagui Mikhail for his technical support.

In addition, deep thankfulness to my friends, Dr. Lilia Forte, Francis Fisk, Erik michon and JQ Li.

Finally I would like to express my heartfelt appreciation to my family, especially to my mother, Prof. Hongxing Dong, who has given me strength and wisdom to overcome all the difficulties. Happy belated birthday. 


\section{Table of Contents}

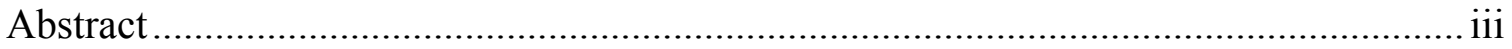

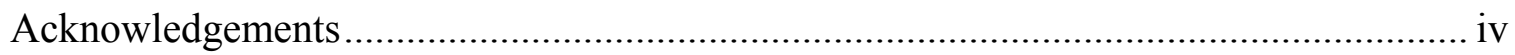

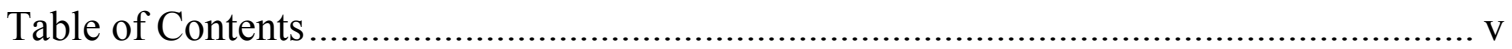

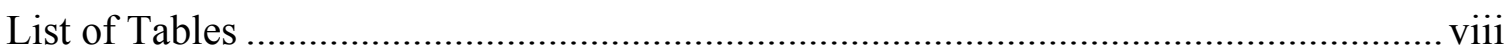

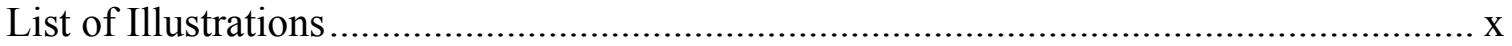

List of Symbols and Abbreviations............................................................................

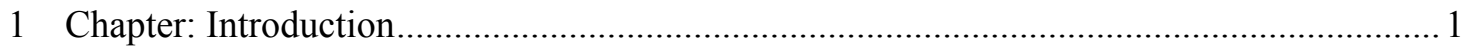

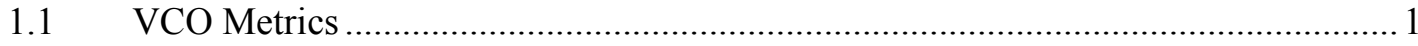

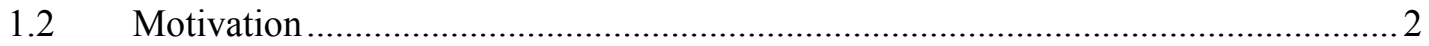

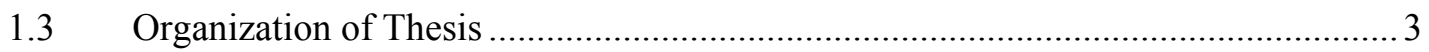

2 Chapter: Reviews on VCO Theory ........................................................................

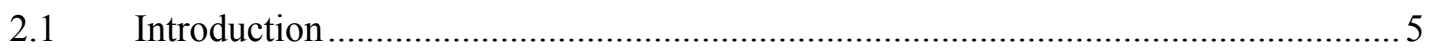

$2.2 \quad$ Reviews on VCO Theory …………………………..................................

2.2.1 Barkhausen Criteria ………………………………....................................

2.2.2 Negative Resistance Approach ……………………………………………...

2.2.3 Leeson's Empirical Expression …………………………………………...

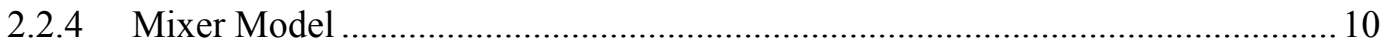

2.2.5 Impulse Sensitivity Function ...................................................................... 12

2.3 VCO Topology Examples from Literatures …………………………………….... 14

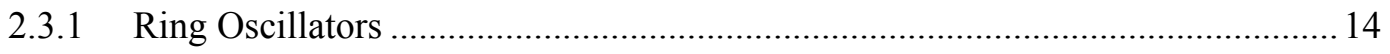

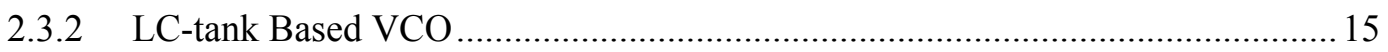

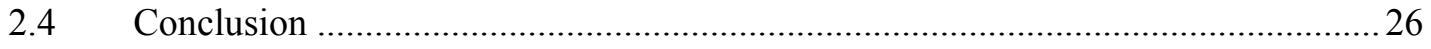




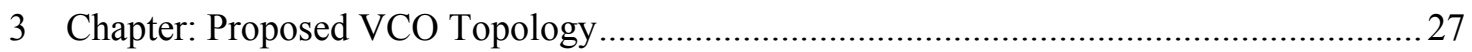

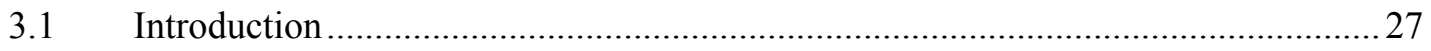

3.2 Study on Relation between Phase Noise and Harmonics .......................................27

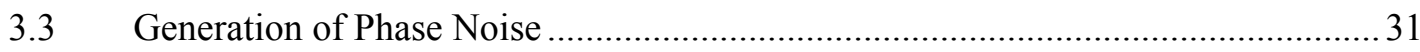

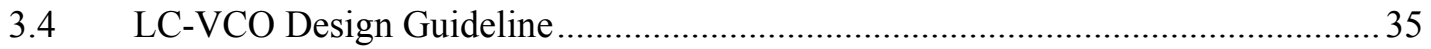

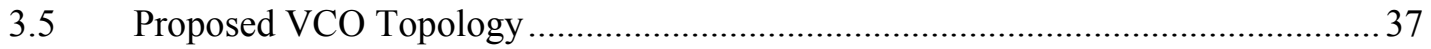

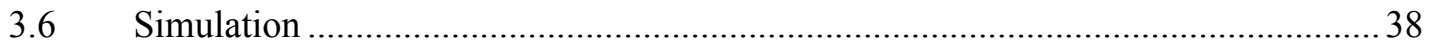

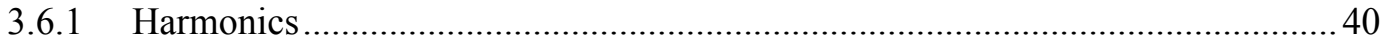

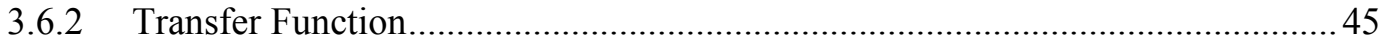

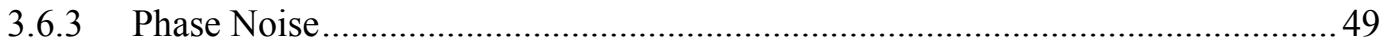

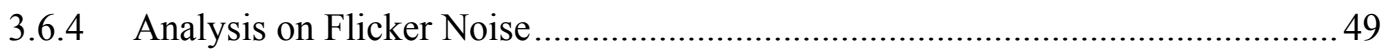

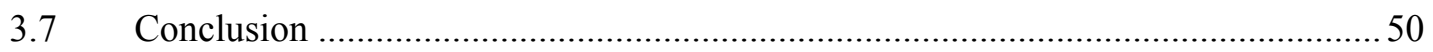

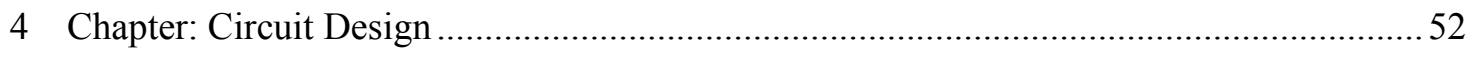

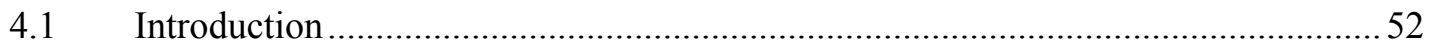

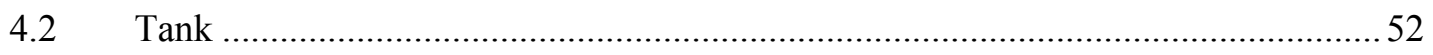

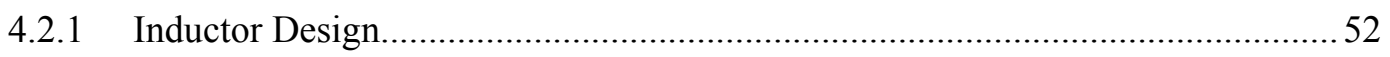

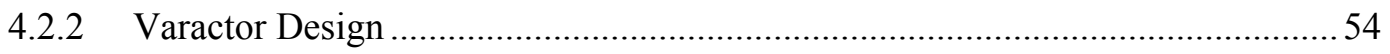

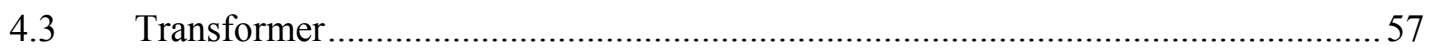

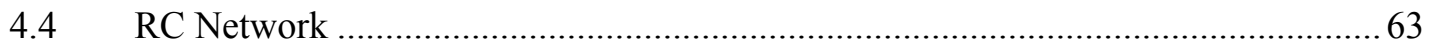

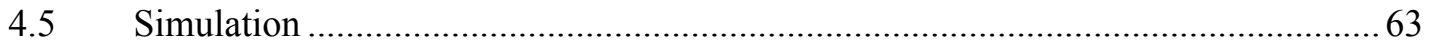

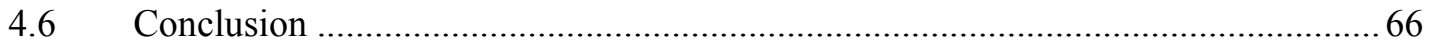

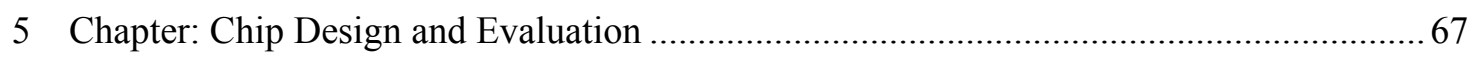

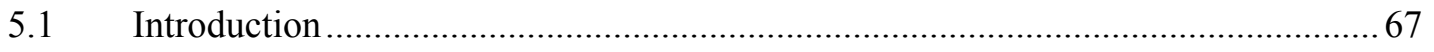

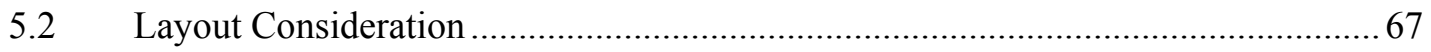

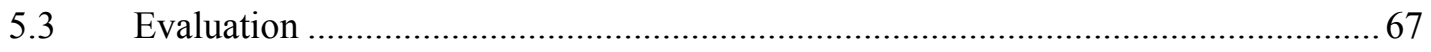

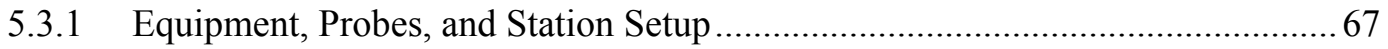


5.3.2 Measurement and Failure Analysis............................................................ 70

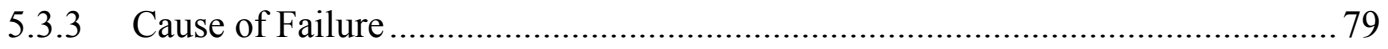

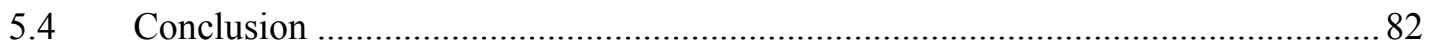

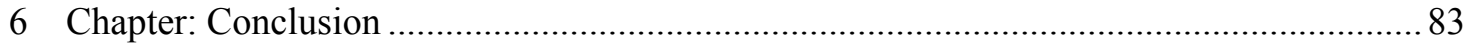

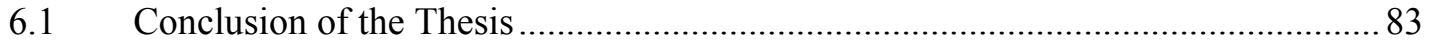

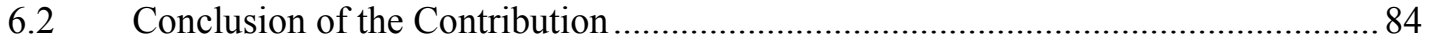

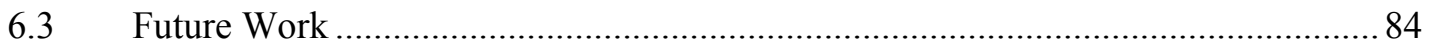

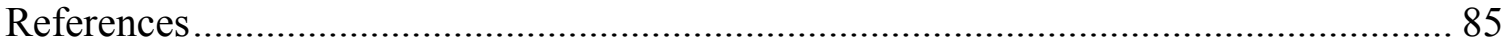

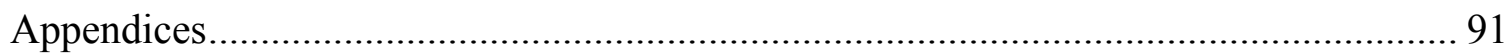




\section{List of Tables}

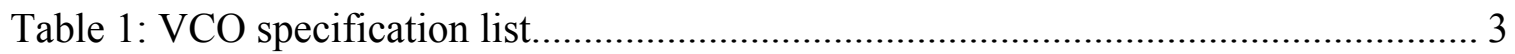

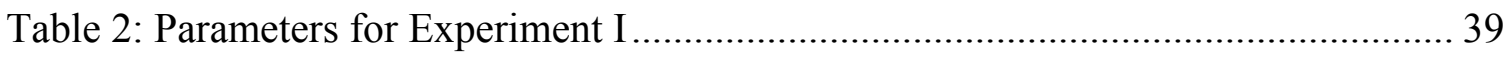

Table 3: Parameters for Experiment II........................................................................... 39

Table 4: Simulation results for Harmonics for Experiment I .......................................... 41

Table 5: Simulation results for Harmonics for Experiment II ............................................ 41

Table 6: Simulation results for K value for Experiment I ............................................. 46

Table 7: Simulation results for K value for Experiment II ............................................. 46

Table 8: Simulation results for phase noise performance for Experiment I ..................... 47

Table 9: Simulation results for phase noise performance for Experiment II .................... 47

Table 10: Simulation results for phase noise performance for Experiment I under

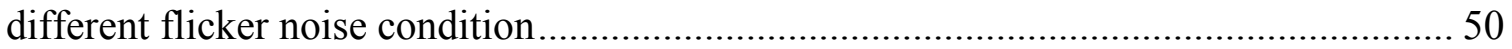

Table 11: Comparison between different layout methods for on-chip transformers ........ 58

Table 12: Parameters for transformer1 and transformer2 (Square Spiral) ....................... 60

Table 13: Parameters for the design symmetrical transformer .......................................... 61

Table 14: Parameters for differential dual layer inductors .............................................. 62

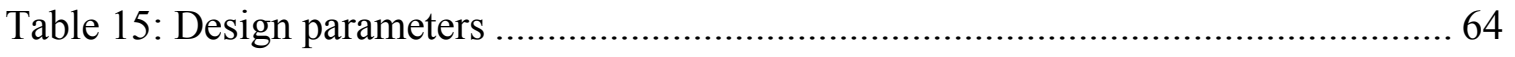

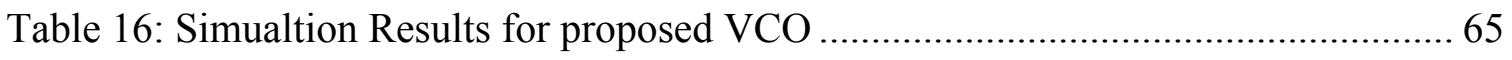

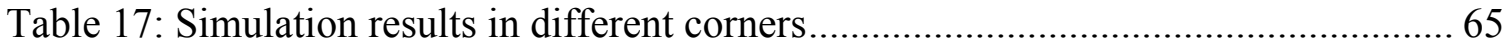

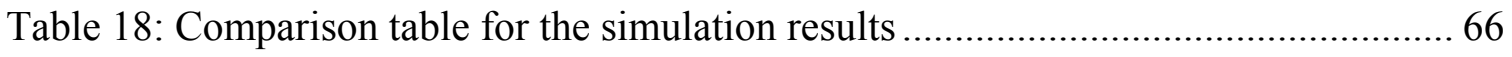

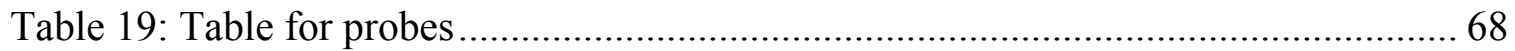

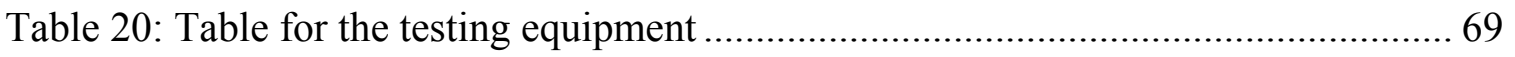

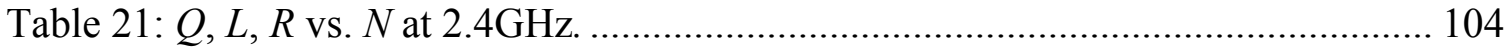




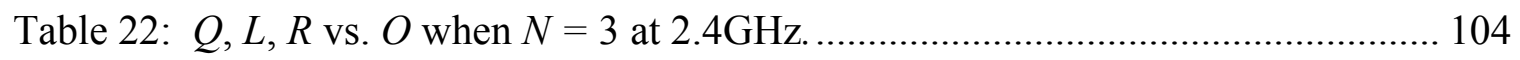

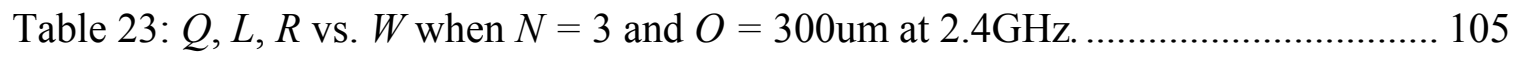

Table 24: $Q, L, R$ vs. $S$ when $N=3, O=300 \mathrm{um}$ and $W=8.5 \mathrm{um}$ at $2.4 \mathrm{GHz} \ldots \ldots \ldots \ldots . . . .105$ 


\section{List of Illustrations}

Figure 1.1: Block diagram of RF front end in a typical heterodyne radio transceiver. ...... 2

Figure 1.2: Block diagram for a typical PLL system ................................................. 2

Figure 2.1: Block diagram of feedback oscillator circuit ....................................... 6

Figure 2.2: Negative resistance model for oscillators.............................................. 7

Figure 2.3: Schematic of conventional differential LC-VCO....................................... 7

Figure 2.4: (a) One-port model for LC-VCO; (b) Small signal model for LC-VCO. ....... 8

Figure 2.5: (a) Noise at input of differential pair modulates instants of zero crossing. (b)

Output current consists of square wave and random noise pulses.............................. 12

Figure 2.6: (a) Impulse injected at the peak. (b) Impulse injected at the zero crossing. .. 13

Figure 2.7: Typical ISF for (a) LC-VCOs and (b) ring oscillators............................... 13

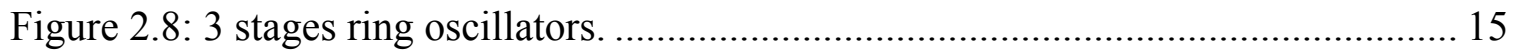

Figure 2.9: Time domain waveforms for conventional LC-VCO................................ 16

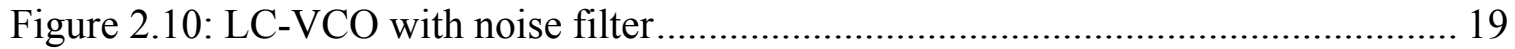

Figure 2.11: (a) LC-VCO based on transformer feedback. (b) Class-C VCO.................. 20

Figure 2.12: Time domain waveforms for class-C VCO ........................................ 21

Figure 2.13: Time domain waveforms for VCO using tail current shaping technique..... 24

Figure 2.14: Schematic of the fabricated VCO2 core and differential bipolar peak

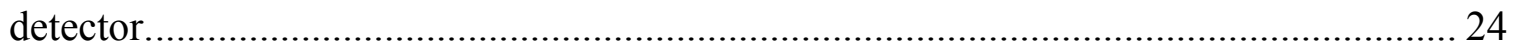

Figure 2.15: Dual-Conduction class-C VCO ....................................................... 25

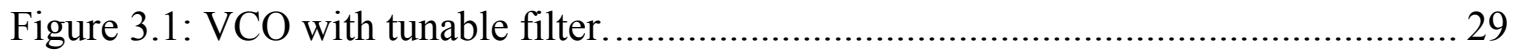

Figure 3.2: Simulation results for the phase noise performance with different harmonics

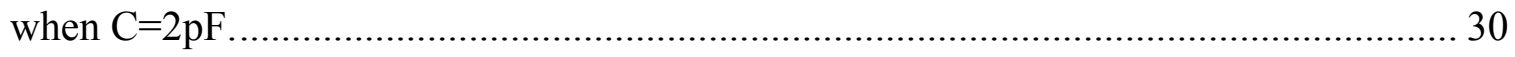


Figure 3.3: Simulation results for the phase noise performance with different harmonics when $\mathrm{C}=1 \mathrm{pF}$ 30

Figure 3.4: Simulation results for the phase noise performance with different harmonics when $\mathrm{C}=0.5 \mathrm{pF}$ 31

Figure 3.5: Black box model for oscillators [37] ................................................. 32

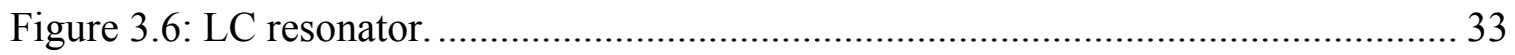

Figure 3.7: Impedance of a LC-VCO (example)................................................. 33

Figure 3.8: Conversion of noise to phase noise ..................................................... 34

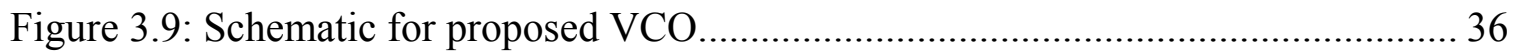

Figure 3.10: Topology for (a) VCO1 (b) VCO2 (c) VCO3 (Proposed). (d)(e)(f) show voltage waveform in time domain for three VCOs. $(\mathrm{g})(\mathrm{h})(\mathrm{i})$ show injected current in time

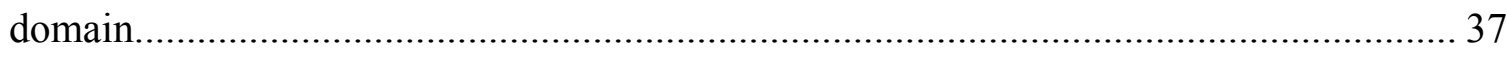

Figure 3.11 Harmonic simulation of (a) VCO1 (b) VCO2 (c) VCO3 for Experiment I. . 43 Figure 3.12: Harmonic simulation of (a) VCO1 (b) VCO2 (c) VCO3 for Experiment II.44 Figure 3.13: Comparison of transfer functions for (a) Experiment I (b) Experiment II... 47

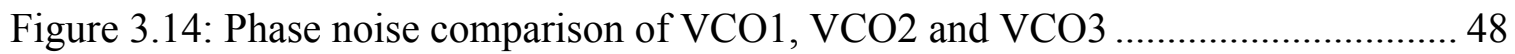
Figure 3.15: Phase noise comparison of $\mathrm{VCO} 1, \mathrm{VCO} 2$ and $\mathrm{VCO} 3$ when flicker noise is introduced. 50

Figure 4.1: Single-end planar spiral inductor layout methods. (a) Square (b) Hexagonal (c)

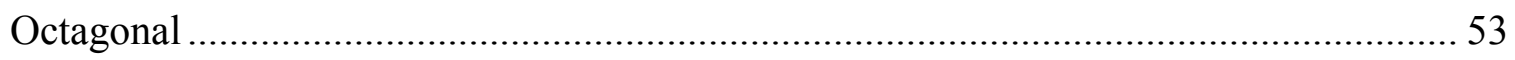

Figure 4.2: Differential symmetric layout method. .................................................. 53

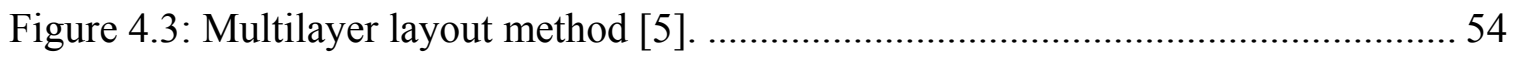

Figure 4.4: Simulation results for single-end inductors.......................................... 55 
Figure 4.5: Side view of MOS varactor

Figure 4.6: Varactors working in (a) Inversion region (b) Depletion region (c)

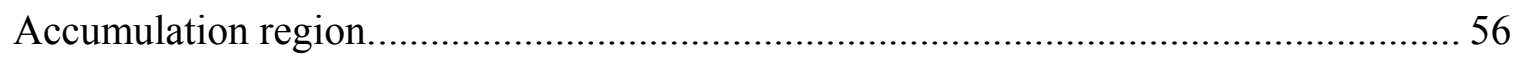

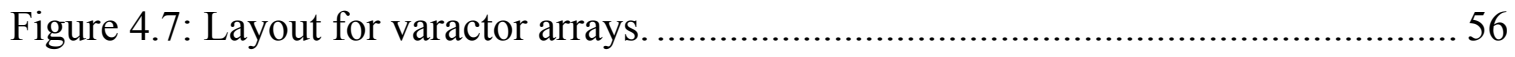

Figure 4.8: Simualtion results for the characterization of varactor arrays...................... 57

Figure 4.9: Layout method for on-chip transformers. (a) Tapped (b) interleaved (c)

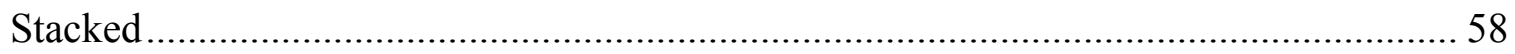

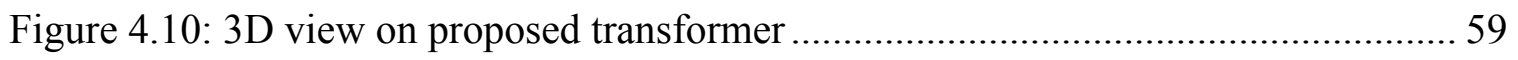

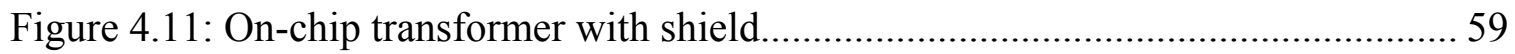

Figure 4.12: Layout method for the proposed transformer ...................................... 59

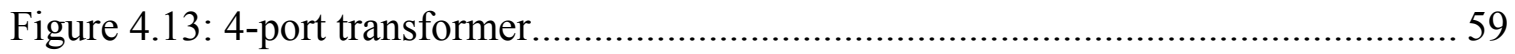

Figure 4.14: Simulation results for Transformer1 and Transformer2 using ASITIC ....... 60

Figure 4.15: Schematic for proposed transformer using ASITIC ............................... 61

Figure 4.16: Simulation results for the differential inductance of dual layer inductors. .. 62

Figure 4.17: Simulation results for the coupling coefficient of proposed transformer..... 62

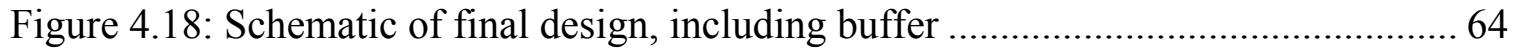

Figure 4.19: Simualtion result for the phase noise performance of the proposed VCO at

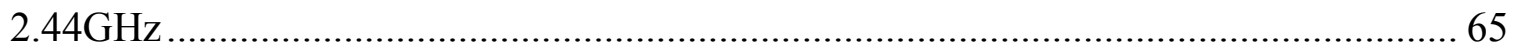

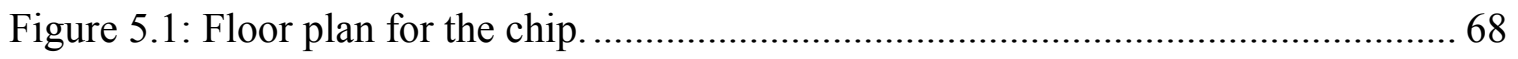

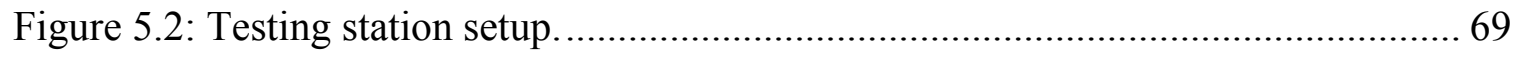

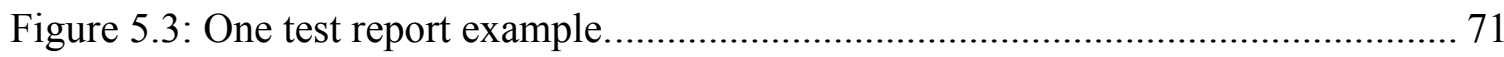

Figure 5.4: Simplified diagram for the proposed VCO ....................................... 72 
Figure 5.5: (a) Phase Noise (b) Transient response (0-10ns) (c) Transient response (0-1us) 73

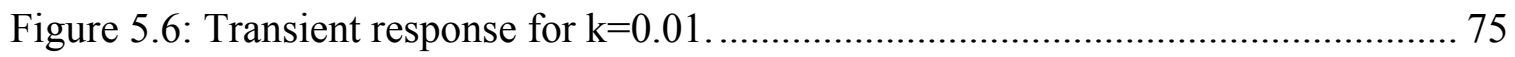

Figure 5.7: (a) Phase Noise (b) Transient response (0-10ns) (c) Transient response (0-1us) 76

Figure 5.8: Transient response for VCO with extra pad capacitance. 77

Figure 5.9: (a) Phase Noise (b) Transient response (0-10ns) (c) Transient response (0-1us)

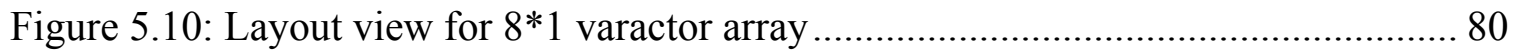

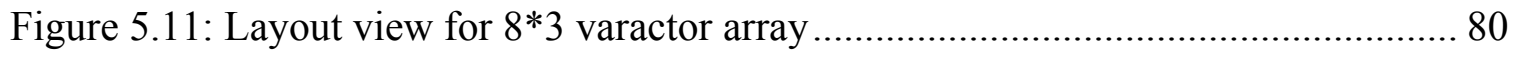

Figure 5.12: Expecting layer MQ for the entire varactor array $(32 * 15$ units $) \ldots \ldots \ldots \ldots \ldots \ldots . . . . . . .11$

Figure 5.13: Wrong layout view for layer MQ ................................................... 81

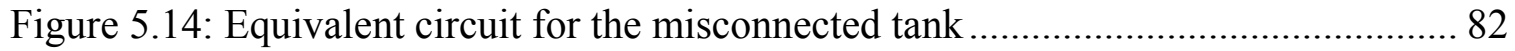

Figure A.1: Block diagram for typical negative feedback system ............................... 92

Figure A.2: Testing signal placement. (a) Voltage (b) Current ................................... 92

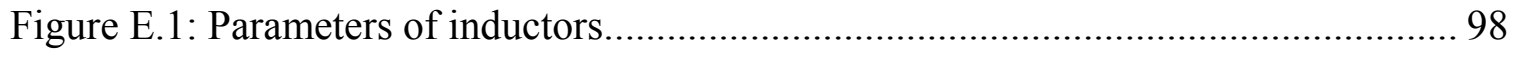

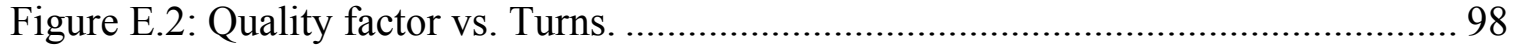

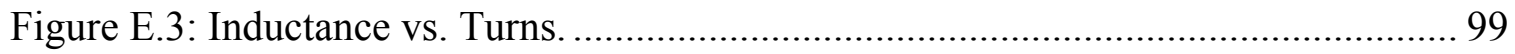

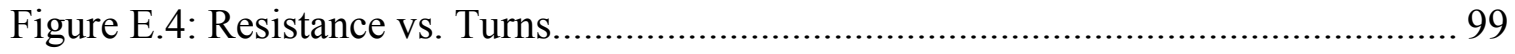

Figure E.5: Quality factor vs. Outer dimension.................................................. 100

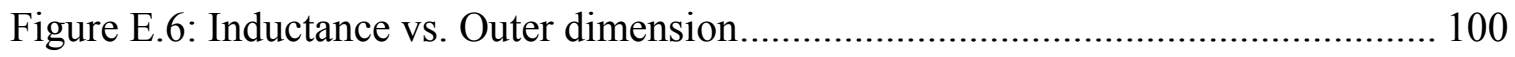

Figure E.7: Resistance vs. Outer dimension.................................................... 101 


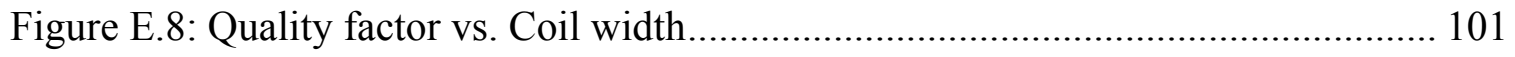

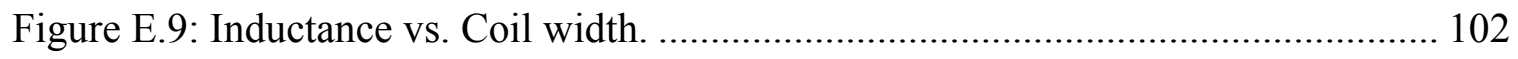

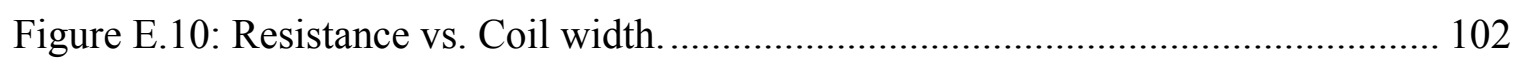

Figure E.11: Quality factor vs. Space ................................................................ 103

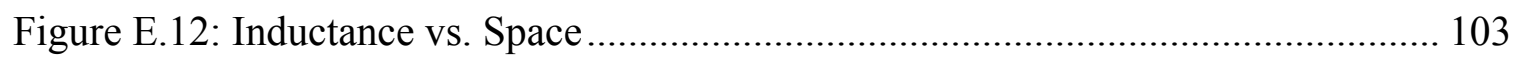

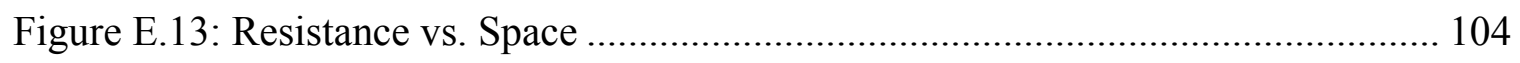




\section{List of Symbols and Abbreviations}

$\begin{array}{ll}\gamma & \text { Noise factor of field }=\text { effect transistor } \\ \theta & \text { Phase } \\ \omega & \text { Angular frequency } \\ f & \text { Frequency } \\ F & \text { Noise factor } \\ g_{m} & \text { Transconductance of transistor } \\ G & \text { Gain of Transfer Function } \\ G_{m} & \text { Transconductance of differential pair } \\ H & \text { Function of feedback part } \\ j & \text { Imaginary unit }\left(j^{2}=-1\right) \\ k_{B} & \text { Boltzman constant }\left(1.3807 \times 10^{-23}[\mathrm{~J} / \mathrm{K}]\right) \\ \mathcal{L} & \text { Noise power per Hertz } \\ P & \text { Power } \\ q & \text { Charge of electron } \\ Q & \text { Quality factor } \\ T & \text { Absolute temperature }[\mathrm{K}]\end{array}$

$\begin{array}{ll}\text { AC } & \text { Alternating Current } \\ \text { CMOS } & \text { Complementary Metal Oxide Semiconductor (FET) } \\ \text { DC } & \text { Direct Current } \\ \text { FET } & \text { Field-Effect Transistor } \\ \text { ISF } & \text { Impulse Sensitivity Function } \\ \text { MOS } & \text { Metal Oxide Semiconductor } \\ \text { NMOS } & \text { N-Channel Metal Oxide Semiconductor (FET) } \\ \text { PLL } & \text { Phase-Locked Loop } \\ \text { PMOS } & \text { P-Channel Metal Oxide Semiconductor (FET) } \\ \text { RF } & \text { Radio Frequency } \\ \text { THD } & \text { Total Harmonic Distortion }\end{array}$




\section{Chapter: Introduction}

With the rapid development of the wireless communication industry, more available channels are demanded. As a result, phase noise requirements in the local reference signal in transceiver and receiver systems (Figure 1.1) are becoming more stringent. Usually the reference signal is generated by a frequency synthesizer [2- 4], of which there are three types: direct analog synthesizers, direct digital synthesizer and indirect digital synthesizers. Indirect digital synthesizers, or the so-called phase-locked loop (PLL), are implemented by locking the phase of the voltage-controlled oscillator (VCO) to the reference signal.

In typical PLL architecture, the VCO is the main component for determining the phase noise performance and power consumption of the entire system (see Figure 1.2). In the past, the VCO was fabricated in gallium arsenide (GaAs) technology or bipolar junction transistors (BJT) for higher performance. Nowadays, as the integrated circuits converge into complementary metal-oxide semi-conductors (CMOS), studies and designs around high-performance CMOS VCOs have become very important.

\subsection{VCO Metrics}

Table 1 lists the specifications and requirements for a VCO. Among them, center frequencies, tuning range, phase noise, and power consumption are the key metrics. Center frequency and tuning range differ from the applications. Usually, center frequency may be influenced by temperature and other conditions, thus a wide tuning range is desirable [5].

Designing a low-phase noise VCO is a major direction in VCO research. Many theory and topologies have emerged with the aim of achieving better phase noise [6-26]. With greater numbers of portable wireless devices developed, low-power VCO design has become another major research direction. Obviously, to achieve low power, the most direct method is to reduce supply voltage. However, reducing the supply voltage will inevitably increase phase noise. Therefore, it is extremely important to find a 


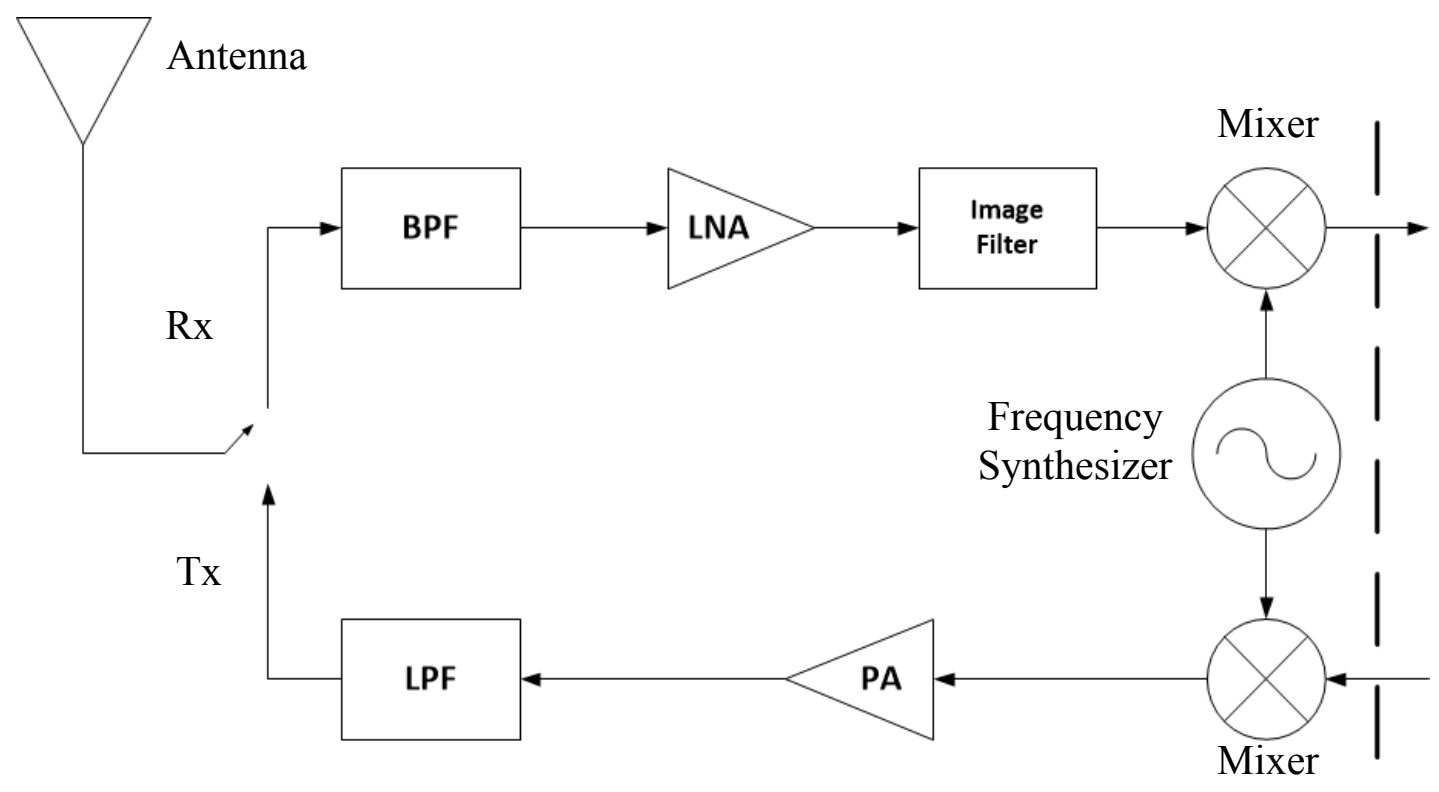

Figure 1.1: Block diagram of RF front end in a typical heterodyne radio transceiver.

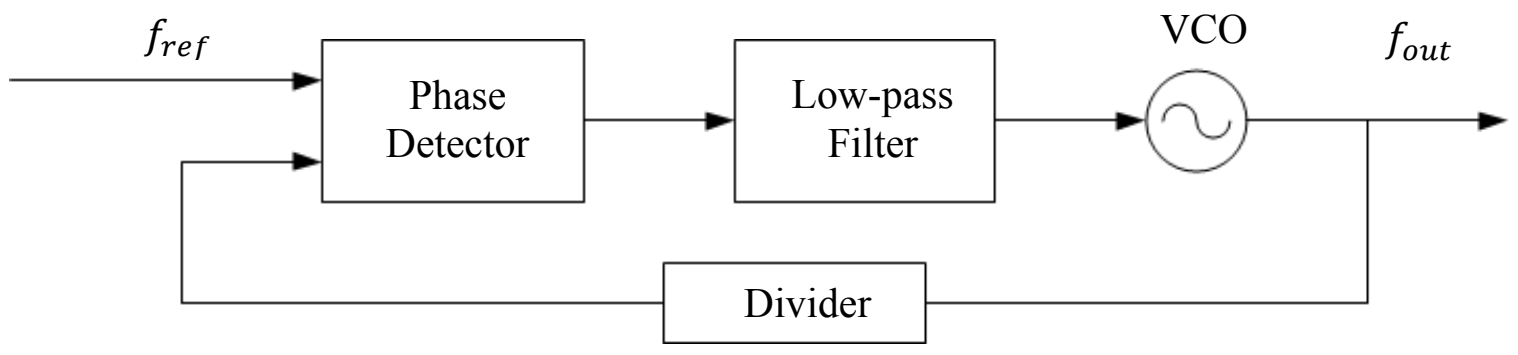

Figure 1.2: Block diagram for a typical PLL system

solution for achieving high-phase noise performance under a limited voltage supply and power budget.

\subsection{Motivation}

Many efforts have been made to study the generation of phase noise. Various models and approaches have been put forward to predict the phase noise, from Leeson's empirical equation [27] in 1966 to Hajimiri's Impulse Sensitive Function approach [13] in 1999. However, although accurate in predicting the phase noise, these theory are less practical 
Table 1: VCO specification list

\begin{tabular}{c|c}
\hline Specifications & Unit \\
\hline Center Frequency & $\mathrm{GHz}$ \\
Tuning Range & $\mathrm{MHz}$ \\
Phase Noise & $\mathrm{dBc} / \mathrm{Hz} @$ offset $(\mathrm{Hz})$ \\
Power Consumption & $\mathrm{mW}$ \\
Supply Voltage & $\mathrm{V}$ \\
VCO-Gain & $\mathrm{MHz} / \mathrm{V}$ \\
Pulling & $\mathrm{MHz} / \mathrm{load}-\mathrm{spec}$ \\
Pushing & $\mathrm{MHz} / \mathrm{V}$ \\
Area & $\mu m^{2}$ \\
\hline
\end{tabular}

in guiding VCO designs. Therefore it is critical to find a design methodology giving more insight to the actual VCO designing.

Hence, in this work, an attempt to link non-linear behavior to phase noise has been made. From the exploration, non-linear factors that determining the phase noise performance have been found gradually. These factors provide clear insights in VCO designs.

Following the design insights, a VCO topology employs non-linear factors suppression techniques has been proposed. Simulation results showed that the phase noise performance is highly increased in the proposed circuit. For further verification, a 2.4GHz VCO example has been designed and fabricated in IBM $130 \mathrm{~nm}$ process using the proposed topology.

\subsection{Organization of Thesis}

This thesis starts with a review in VCO theory and topologies in Chapter 2. The theory includes both linear and non-linear approaches. Topologies cover the review of the circuits from previous literatures.

In Chapter 3, an analysis of phase noise generation is first presented, followed by a proposal of VCO topology aimed at reducing phase noise using non-linear factors 
suppression techniques. Simulation results comparison among the proposed topologies and other two references topologies are reported, showing the advantage of the employed techniques.

In Chapter 4, an example circuit using the proposed topology has been designed. Design issues are discussed, including tank, transformer, and overview optimization. Simulation results are shown consequentially.

In Chapter 5, chip design is demonstrated. Measurements setup and failure analysis are included.

In Chapter 6, the thesis is summarized. Contributions are concluded, followed by a discussion of future work. 


\section{Chapter: Reviews on VCO Theory}

\subsection{Introduction}

This chapter first reviews the previous studies on VCO theory, including both linear and non-linear approaches. Barkhausen Criteria, a linear approach in determining the oscillation startup is first reviewed, followed by another linear approach, negative resistance approach. Then Leeson's empirical equation, which gave a general insight of the factors that influence the phase noise performance, is introduced. Darabi's mixer approach gave more insight in the noise factor of the circuit. Finally Hajimiri's non-linear cyclone stationary approach, which is usually referred as Impulse Sensitive Function (ISF), is presented.

In the second part, several circuit examples in recent literatures have been demonstrated, including ring oscillators and LC-tank based oscillators. In this thesis focus are put on LC-VCOs considering their phase noise performance is generally higher than ring-oscillators. Starting with the conventional LC-VCO, another three circuits have been analyzed. Some other techniques and circuits also have been reviewed roughly.

\subsection{Reviews on VCO Theory}

\subsubsection{Barkhausen Criteria}

Barkhausen Criteria [28] is a mathematical formula to determine when a linear electronic circuit will oscillate. The criteria treat the oscillator as a linear positive feedback loop, as shown in Figure 2.1, where the forward gain $G(j \omega)$ represents the voltage transfer function of the amplifier and $H(j \omega)$ is the voltage transfer function of the feedback network. Obviously the transfer function of the complete network is given by

$$
\frac{V_{\text {out }}}{V_{\text {in }}}=\frac{G(j \omega)}{1-G(j \omega) H(j \omega)}
$$




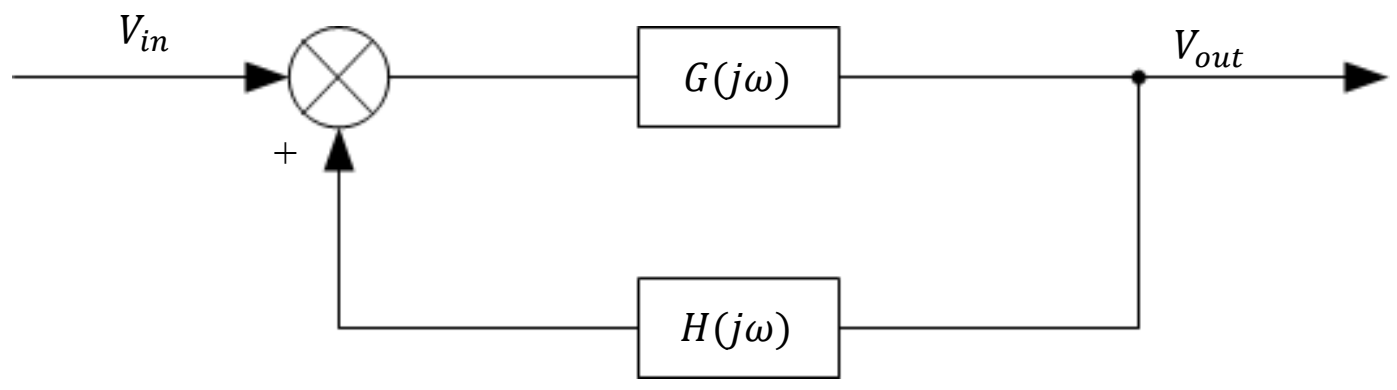

Figure 2.1: Block diagram of feedback oscillator circuit

To make the system unstable, the root of the denominator should be located at the righthand side of the complex axis, given

$$
|G(j \omega)||H(j \omega)| \geq 1
$$

To keep the amplitude constant,

$$
|G(j \omega)||H(j \omega)|=1
$$

For the phase,

$$
\emptyset_{G(j \omega)}+\emptyset_{H(j \omega)}=0+2 \pi k \quad k=1,2 \ldots n .
$$

Equations (3) and (4) represent the well-known Barkhausen Criteria, where $\emptyset_{G(j \omega)}$ and $\emptyset_{H(j \omega)}$ is the phase shift of $G(j \omega)$ and $H(j \omega)$. Note that these criteria pose a necessary condition, but are not sufficient. In some circuits, the criteria are fulfilled, but do not oscillate. In reality, to guarantee the start-up, the loop gain $|G(j \omega)||H(j \omega)|$ is usually made larger than 1 , leading to increase of the amplitude. Then, the gain will fall back to 1 as a result of the nonlinearity of the circuit. 


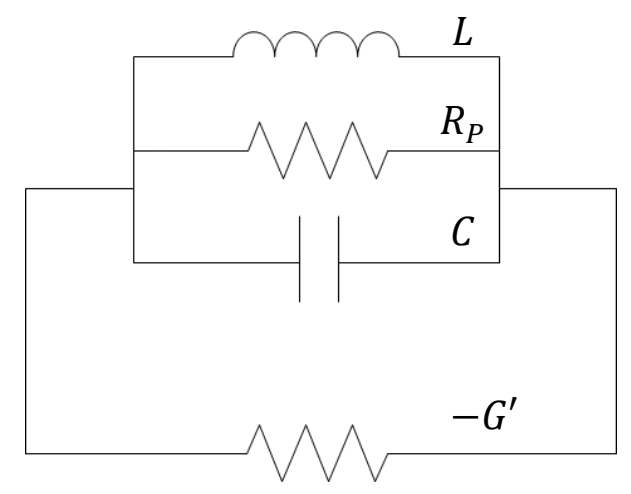

Figure 2.2: Negative resistance model for oscillators

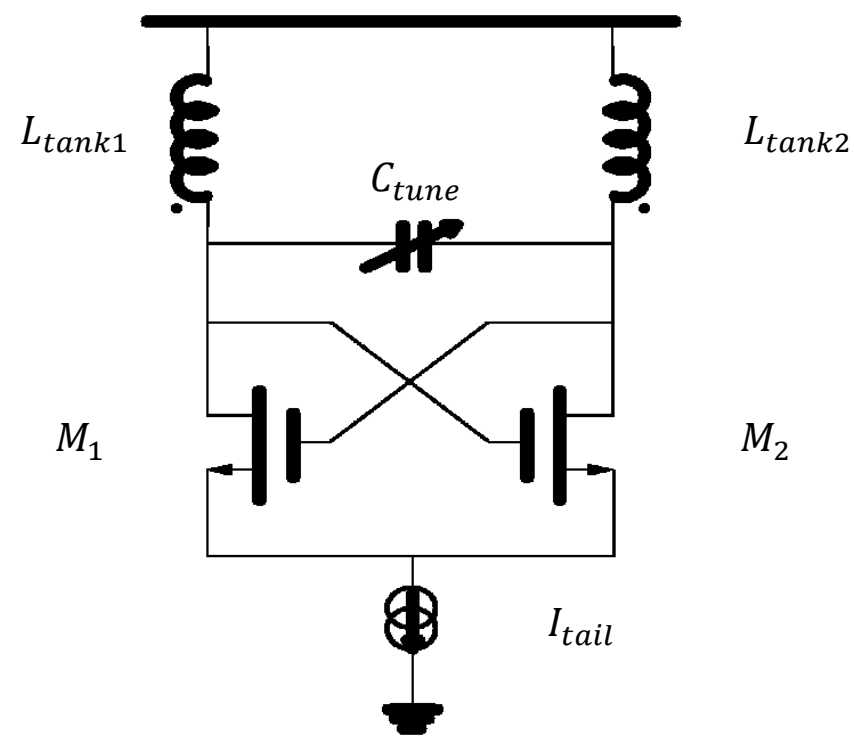

Figure 2.3: Schematic of conventional differential LC-VCO

\subsubsection{Negative Resistance Approach}

This approach is widely used in LC tank-based oscillators. In actual LC tanks, the oscillations will die out because of parasitic resistance as the resistance consumes power and causes energy loss. Hence, to maintain the oscillation, the active network should generate a negative conductance, $-G^{\prime}$, to cancel the conductance $G$ from the tank (see Figure 2.2).

As the calculation of $-G^{\prime}$ depends on the topology, here the conventional LC$\mathrm{VCO}$ is taken as an example (Figure 2.3). Consider the VCO as a circuit consisting of a port (tank) and a negative resistance network shown in Figure 2.4(a). The equivalent 


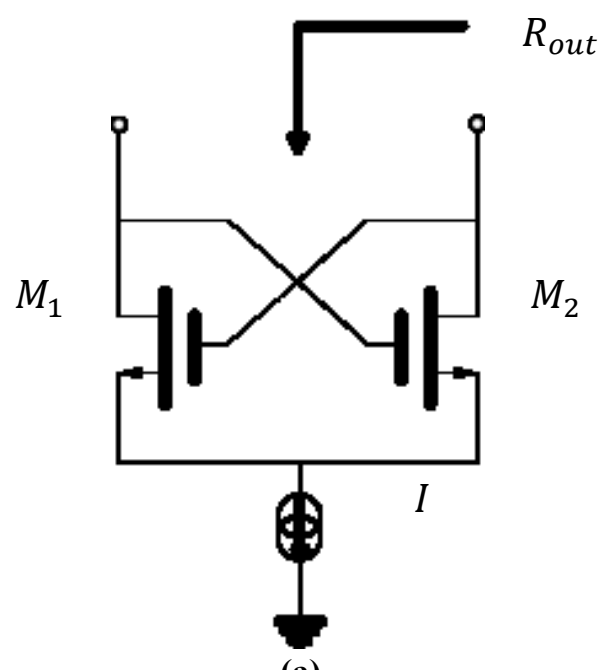

(a)

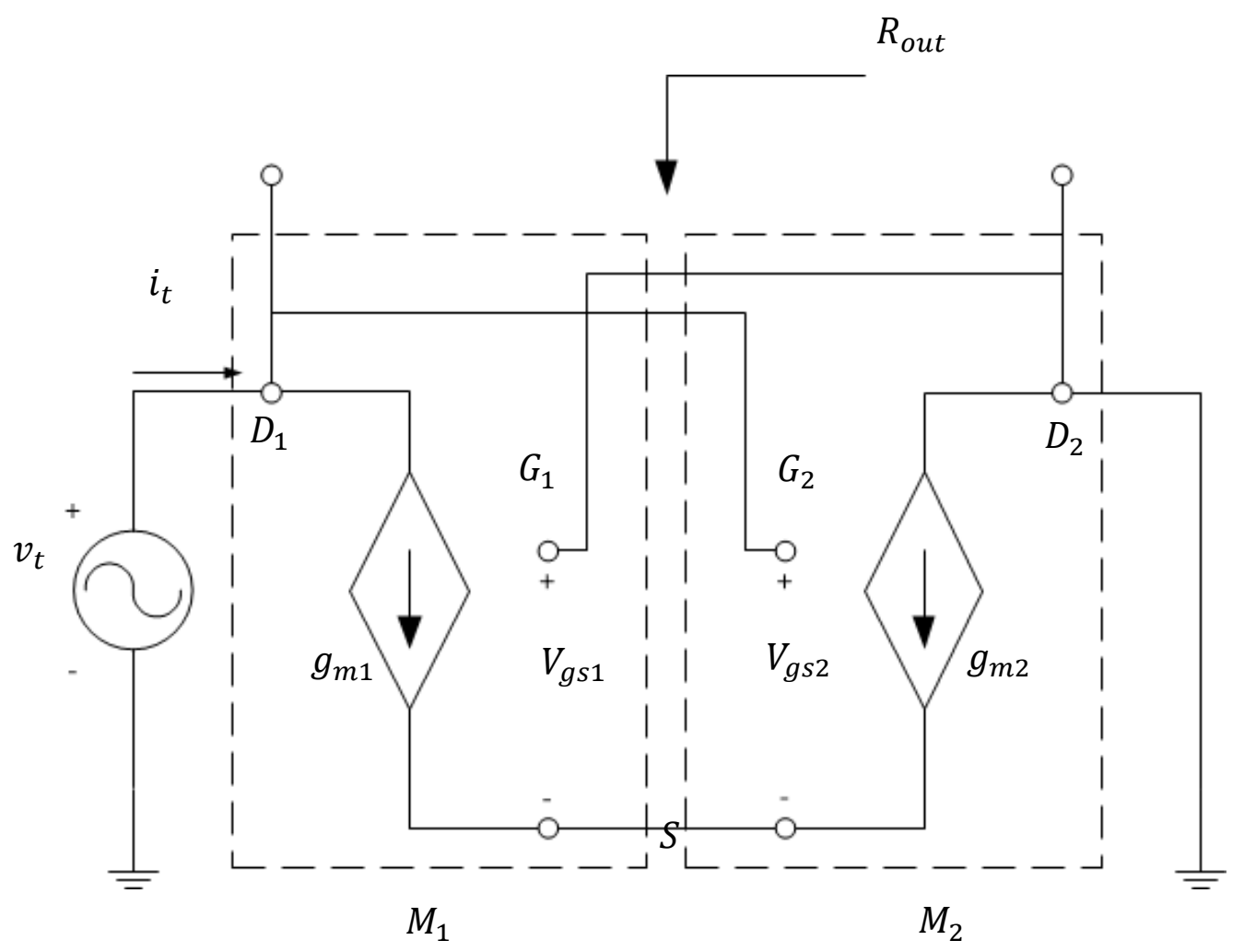

(b)

Figure 2.4: (a) One-port model for LC-VCO; (b) Small signal model for LC-VCO.

small signal circuit is shown in Figure 2.4(b). The dependence of the signal quantities on time will be dropped in order to simplify the analysis of this circuit. 
Given the voltage across $v_{t}$

$$
v_{t}=v_{g s 2}-v_{g s 1}
$$

and the current flow through the transistors $i_{t}$

$$
i_{t}=g_{m 1} v_{g s 1}=-g_{m 2} v_{g s 2}
$$

where $g_{m}$ is the transconductance of the transistors, $R_{\text {out }}$ can be given by

$$
R_{\text {out }}=\frac{v_{t}}{i_{t}}=-\frac{1}{g_{m 1}}-\frac{1}{g_{m 2}}
$$

In typical design $g_{m 1}=g_{m 2}$, given

$$
R_{\text {out }}=\frac{v_{t}}{i_{t}}=-\frac{2}{g_{m 1}}
$$

Equation (9) provides insights in choosing appropriate dimensions of active devices. It should be noted that $g_{m 1}$ and $g_{m 2}$ are the average transconductance.

\subsubsection{Leeson's Empirical Expression}

In 1966, Leeson [27] put forward a well-known empirical expression (10) for the singlesideband phase-noise spectrum of oscillators,

$$
\mathcal{L}(\Delta f)=10 \log \left[\frac{1}{2}\left(\left(\frac{f_{0}}{2 Q_{L}}\right)^{2}+1\right)\left(\frac{f_{c}}{\Delta f}+1\right)\left(\frac{F k_{B} T}{P_{S}}\right)\right]
$$

where $f_{0}$ is the output frequency, $Q_{L}$ is the loaded quality factor of the tank, $f_{c}$ is the $1 /$ $f$ corner frequency, $\Delta f$ is the offset from the output frequency, $F$ is the noise factor, $k_{B}$ is 
Boltzman's constant, $T$ is the absolute temperature in Kelvins, and $P_{S}$ is the output power of the oscillator.

This empirical expression gives two ways of reducing the phase noise, whether increasing $Q_{L}$ or increasing $P_{S}$. To maximize $Q_{L}$, one should employ a high $Q$ tank. Because

$$
P_{s} \propto V_{\text {peak }}^{2}
$$

The most efficient way to improve $P_{S}$ is to increase $V_{\text {peak }}$, which is the maximal voltage across the tank. Usually $V_{\text {peak }}$ is limited by the supply voltage, but also depends on the topologies.

With

$$
\begin{gathered}
Q_{L} \approx \omega L_{s} / R_{S} \\
\boldsymbol{f}_{\boldsymbol{c}} \gg \Delta \boldsymbol{f} \\
P_{S}=V_{\text {peak }}^{2} /\left(2 R_{S}\right)
\end{gathered}
$$

equation (10) can be simplified to:

$$
\mathcal{L}(\Delta f)=10 \log \left[\left(\frac{F k_{B} T}{P_{S}}\right)\left(\frac{f_{c}}{\Delta f}\right)^{2}\right] .
$$

Note that equation (15) only describes the $1 / f^{2}$ portion of the phase noise.

\subsubsection{Mixer Model}

In 2000, Darabi proposed a simple model [29] for noise in mixers, showing that the model of switching pairs is sufficient to explaining all frequency translations of noise. In 
[25], Rael used the model to explain the noise in conventional differential LC-VCOs (see Figure 2.3).

The model treats the transistors as switching pairs. Assume that the sinusoidal output waveform $v_{o}$ is accompanied by noise $v_{n}$. Suppose that the switching pair can be fully turned on with a small amount of voltage around the zero crossing, hence the pulsewidth modulated current can be decomposed into the original periodic-square wave and random-width pulses with constant height $2 I_{\text {tail }}$ (Figure 2.5). These pulses can be viewed as a train of impulses with a double-oscillation frequency sampling the original noise waveform.

The noise source in the circuit forms three parts: tank, differential pair and current source. Hence the phase noise can be given by

$$
\mathcal{L}(\Delta f)=10 \log \left[F \frac{4 k_{B} T R_{p}}{V_{\text {peak }}^{2}}\left(\frac{f_{0}}{2 Q \Delta f}\right)^{2}\right]
$$

with a noise factor $F$, where

$$
F=2+\frac{8 \gamma R_{p} I_{\text {Tail }}}{\pi V_{\text {peak }}}+\frac{8}{9} \gamma g_{m} R_{p} .
$$

Here $R_{p}$ is the equivalent parallel resistance of the tank, $Q$ is the quality factor of the tank, $\gamma$ is the noise factor of a single field-effect transistor (FET) (typically $\gamma \approx \frac{2}{3}$ ), and $g_{m}$ is the transconductance of the FET. Replacing $R_{p}$ by

$$
R_{p}=Q^{2} R_{s}
$$

phase noise can be derived into,

$$
\mathcal{L}(\Delta f)=10 \log \left[\frac{F k_{B} T R_{s}}{V_{\text {peak }}^{2}}\left(\frac{f_{0}}{\Delta f}\right)^{2}\right] .
$$

It can be seen that equation (19) is in accordance with equation (15), which proves 


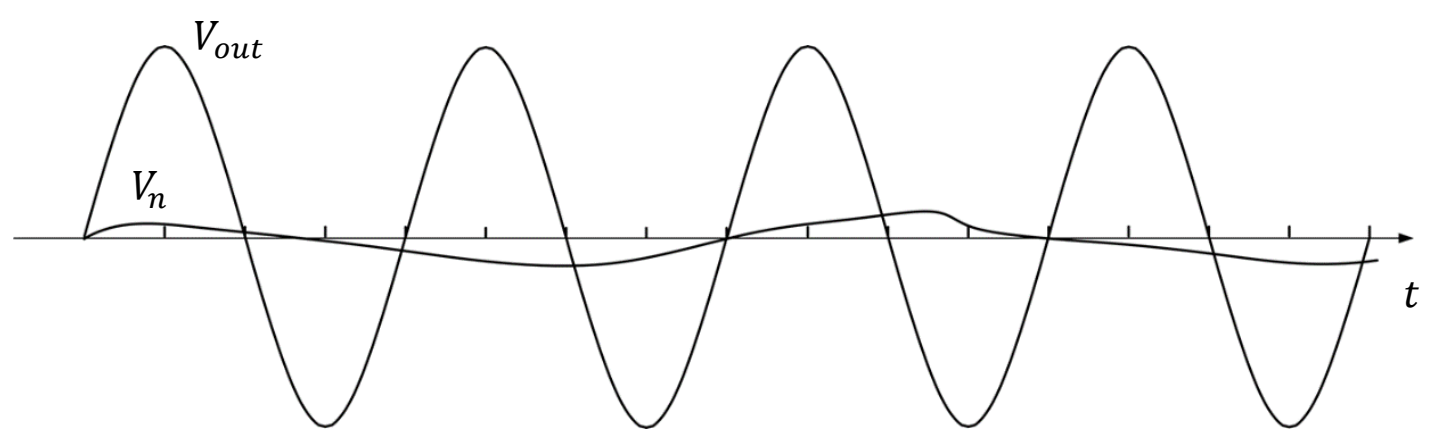

(a)

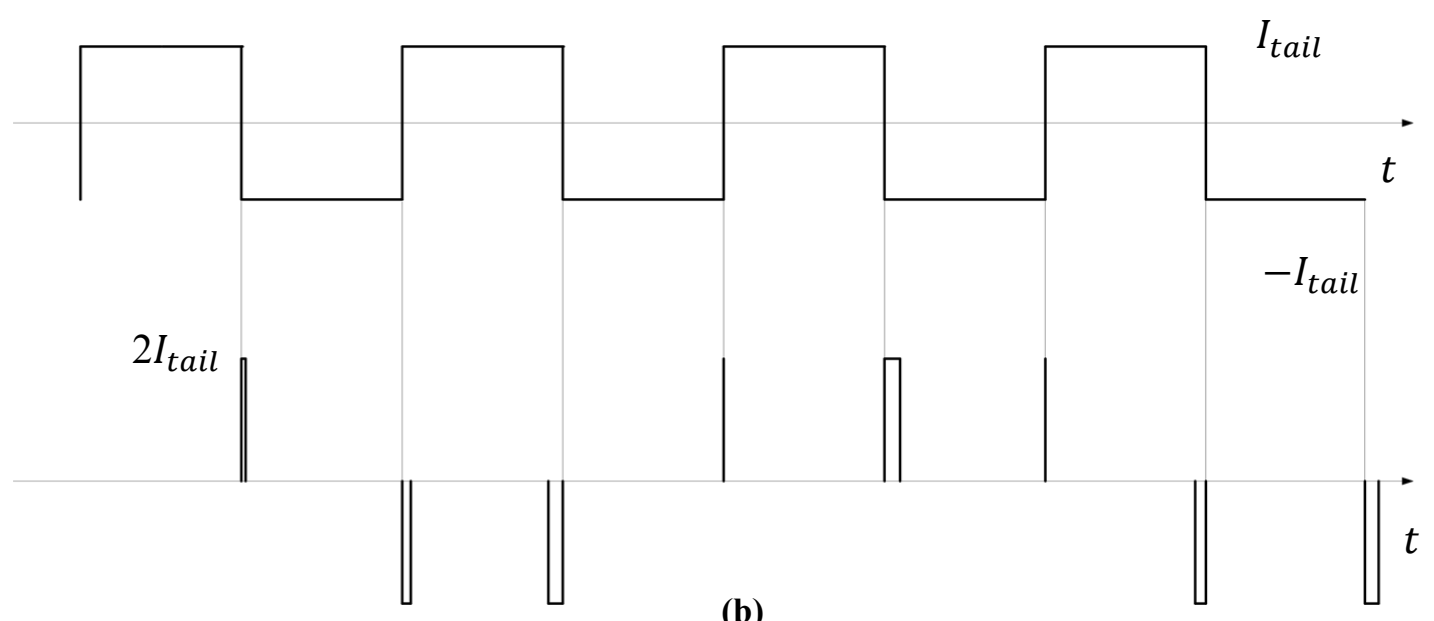

(b)

Figure 2.5: (a) Noise at input of differential pair modulates instants of zero crossing. (b) Output current consists of square wave and random noise pulses.

Leeson's hypothesis. However, this approach gives more insight into the relation between the dimensioning of the active part and the noise factor $F$.

\subsubsection{Impulse Sensitivity Function}

In 1998, Hajimiri proposed a time-variant phase noise model for electrical oscillators [13] [15]. The theory's main idea is that noise sensitivity is time-variant over a period, as illustrated in Figure 2.6. If a current impulse is injected into the voltage waveform at the peak point, it does not cause phase shift, leaving amplitude shift only. If the impulse is 

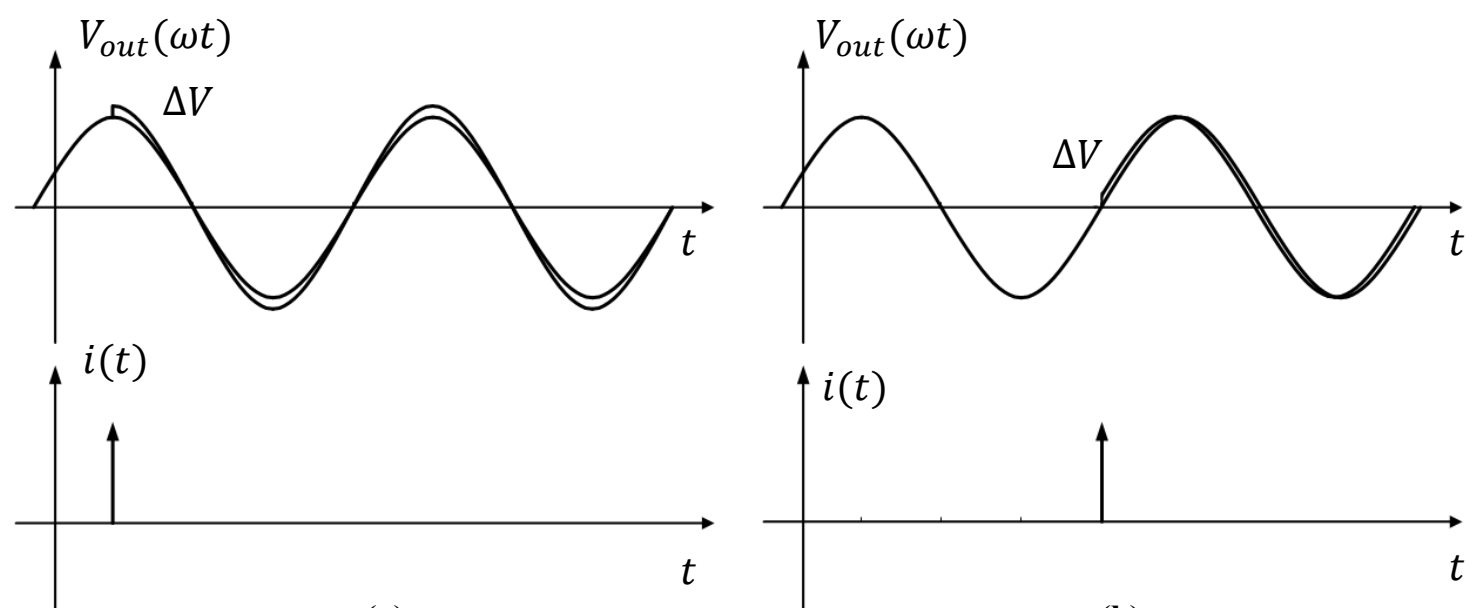

Figure 2.6: (a) Impulse injected at the peak. (b) Impulse injected at the zero crossing.

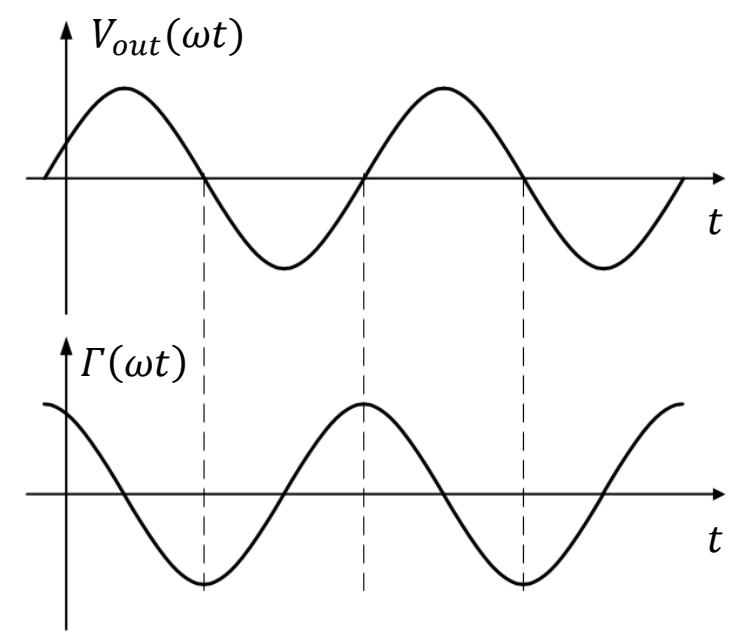

(a)

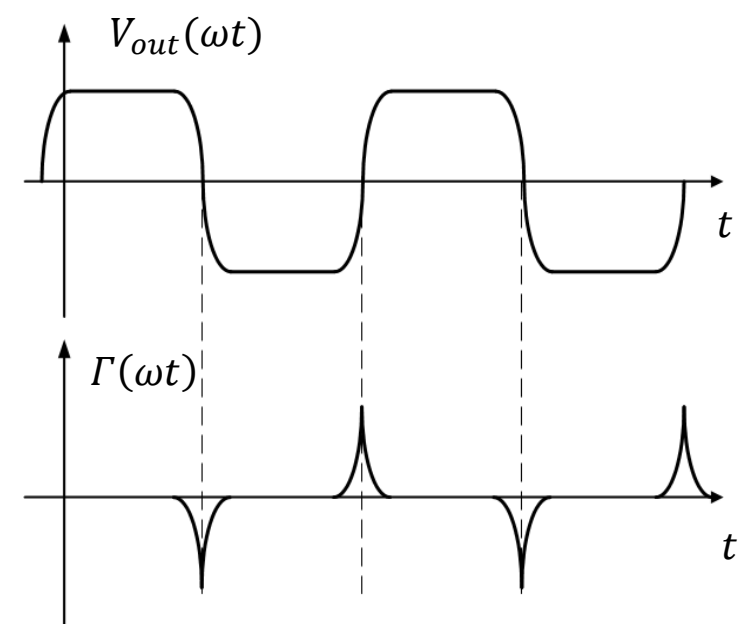

(b)

Figure 2.7: Typical ISF for (a) LC-VCOs and (b) ring oscillators.

injected at the zero crossing point, the noise is transferred only into phase shift, producing no amplitude change. At other points, the impulse causes both amplitude and phase shifts. However, only the phase shift will be transformed into phase noise. Therefore, an impulse sensitivity function (ISF), $\Gamma(\omega t)$, is defined as a dimensionless, frequency, and amplitude-independent periodic function to describe the extent to which phase shifts are caused by applying a unit impulse at $t=\tau$. 
Figure 2.7 shows the ISF for typical LC-tank VCO and ring oscillators. In practice, $\Gamma(\omega t)$ can be approximated as the first derivative of the voltage waveform.

After derivation, the phase noise spectrum of the $1 / f^{2}$ region is:

$$
\mathcal{L}(\Delta \omega)=10 \log \left(\frac{\Gamma_{r m s}^{2}}{q_{\max }^{2}} \frac{\overline{l_{n}^{2}} / \Delta f}{4 \Delta \omega^{2}}\right)
$$

where $\Gamma_{r m s}$ is the rms value of $\Gamma(\omega t), \bar{l}_{n}^{2} / \Delta f$ is the power spectral density of each noise source, $q_{\max }$ is the maximum charges across the tank, $\Delta \omega$ is the offset frequency. $\Gamma_{r m s}$ can be obtained by

$$
\Gamma_{r m s}=\sqrt{\frac{1}{2 \pi} \int_{0}^{2 \pi}|\Gamma(\omega t)|^{2} d \omega t}
$$

$q_{\max }$ can be obtained by

$$
q_{\max }=C V_{\text {peak }}
$$

where $C$ is the capacitance of the tank.

The ISF theory is extremely general and can be applied to all electrical oscillators. The theory accurately predicts the phase noise of a VCO.

\subsection{VCO Topology Examples from Literatures}

\subsubsection{Ring Oscillators}

Ring oscillators are built by a chain of inverters. Each stage offers a phase shift with $180^{\circ}$. To create oscillation, the number of stages should be odd. Figure 2.8 shows a threestage ring oscillator. Ring oscillators are easy to design and integrate, occupying only a small 


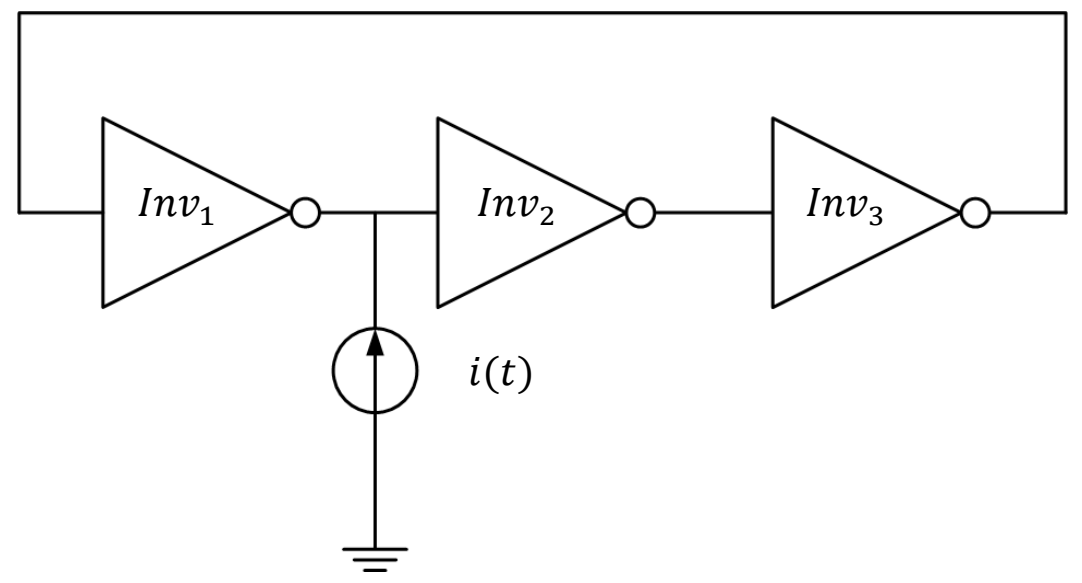

Figure 2.8: 3 stages ring oscillators.

area. Also, they have a very wide tuning range. The main drawback, however, is the highphase noise they produce. Because the ring oscillators form a closed chain, noise inside accumulates, leading to high overall noise [30 -32].

\subsubsection{LC-tank Based VCO}

Here, we only discuss N-type metal-oxide semiconductor logic (NMOS) LC tank-based VCO topologies.

\subsubsection{Conventional LC-VCO}

The most widely used VCO topology is shown in Fig. 2.3. It is also referred to as negative-resistance VCO or cross-coupled differential VCO. Analysis based on the negative resistance model has been presented in chapter 2.2.2.

From the mixer approach, the circuit can be interpreted as using a feedback network consisting of a differential switching pair and FET as the current source. The current source not only provides bias current, but also offers high impedance in series [25]. Both would help to reduce the phase noise.

In time domain, the switching transistors $M_{1}$ and $M_{2}$ are turned on alternatively 


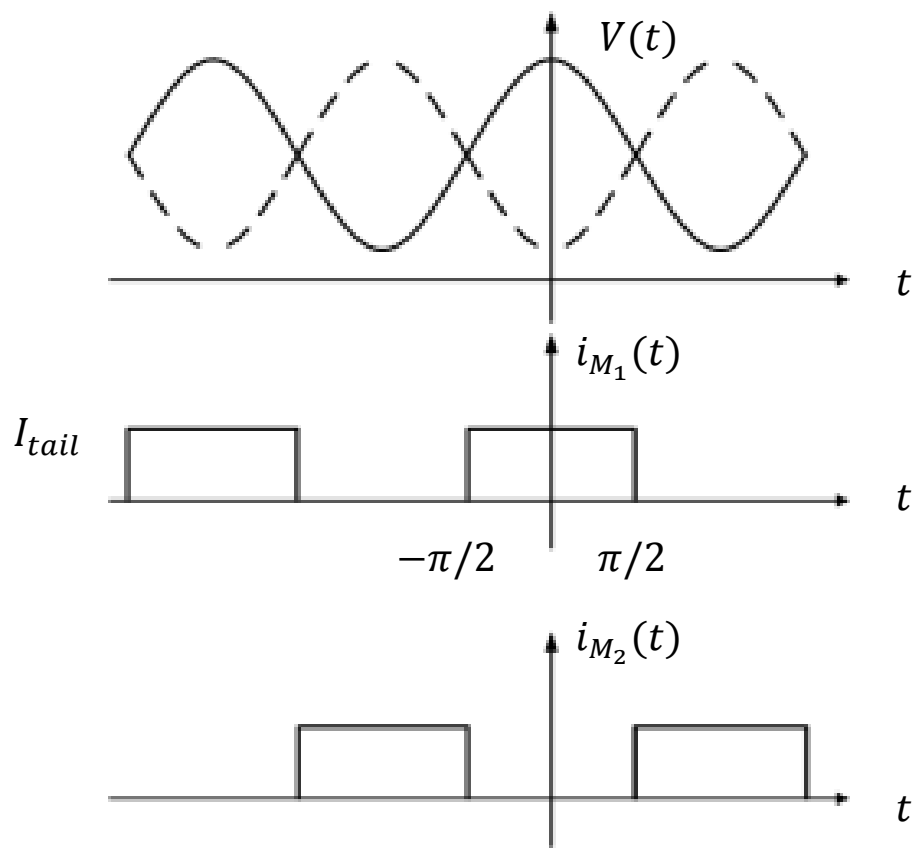

Figure 2.9: Time domain waveforms for conventional LC-VCO.

(see Figure 2.9). The drain currents of $M_{1}$ and $M_{2}$ can be approximated as square wave, which are,

$$
\begin{gathered}
I_{d 1}=I_{\text {tail }} \cdot \sum_{n=-\infty}^{\infty} \prod\left(\frac{\phi-2 n \pi}{\pi}\right) \\
I_{d 2}=I_{\text {tail }} \cdot \sum_{n=-\infty}^{\infty} \prod\left(\frac{\phi-2 n \pi-\pi}{\pi}\right)
\end{gathered}
$$

where $\Pi(x)$ is a single pulse centered at the origin with unity amplitude and duration of 1 , namely

$$
\prod(x)=\left\{\begin{array}{lr}
1, & -0.5 \leq x \leq 0.5 \\
0, & \text { otherwise }
\end{array}\right.
$$

After Fourier transformation, it is easy to obtain the DC component $I_{0}$ and the magnitude of the first harmonic $I_{1}$ at $\omega_{0}$ respectively, which are 


$$
\begin{aligned}
& I_{0}=\frac{1}{2} I_{\text {tail }} \\
& I_{1}=\frac{2}{\pi} I_{\text {tail }} .
\end{aligned}
$$

where $I_{\text {tail }}$ is the average current of the tail current. Since the resonator impedance is low except at frequency around $\omega_{0}$, the single-ended oscillation amplitude can be calculated as

$$
V_{\text {peak }}=\frac{2}{\pi} I_{\text {tail }} R_{p}
$$

where $R_{p}$ is the parallel resistance of the tank.

As it has been mentioned in previous chapter, $\Gamma(\omega t)$ can be approximated as the first derivative of the voltage waveform. Hence for the tank, $\Gamma(\omega t)$ is given by

$$
\Gamma_{\text {tank }, \text { eff }, R M S}^{2}=\frac{1}{2 \pi} \int_{-\pi}^{\pi} \Gamma_{\text {tank }}^{2}(\phi) \alpha^{2}(\phi) d \phi=\frac{1}{2 \pi} \int_{-\pi}^{\pi} \sin ^{2}(\phi) d \phi=\frac{1}{2}
$$

where $\alpha(\phi)$ is the noise modulation function (NMF). The power density of the stationary white noise current induced by the equivalent tank resistance is given as

$$
\overline{l_{R_{p}}^{2}}=4 k_{B} T \frac{1}{R_{p}} \cdot \Delta f
$$

However, since the single-ended switching pair only turns on for half period, the effective noise is then changed to 


$$
\Gamma_{M_{1}, e f f, R M S}^{2}=\frac{1}{2} \Gamma_{\text {tank }, e f f, R M S}^{2}=\frac{1}{4} .
$$

The power spectral density of the thermal current noise of $M_{1}$ can be express as

$$
\overline{l_{M_{1}}^{2}}=4 k_{B} T \gamma g_{m, M_{1}} \Delta f
$$

Similarly, for the constant tail current and power spectral density of the tail current thermal noise,

$$
\begin{gathered}
\Gamma_{\text {tail }, e f f, R M S}^{2}=\frac{1}{2} \Gamma_{\text {tank }, e f f, R M S}^{2}=\frac{1}{4} \\
\overline{l_{\text {tall }}^{2}}=4 k_{B} T \gamma g_{m, \text { tail }} \Delta f .
\end{gathered}
$$

Hence the total phase noise can be calculated as,

$$
\begin{aligned}
\mathcal{L}_{\text {total }}(\Delta \omega)= & 10 \log \left(\frac{\Gamma_{r m s, \text { drain, }, \text { ff }}^{2}}{q_{\text {max }}^{2}} \cdot \frac{\overline{\Delta f}}{2 \Delta \omega^{2}}\right) \\
= & 10 \log \left(\frac{4 k_{B} T\left(1+\frac{1}{2} \gamma g_{m, M_{1}} R_{p}+\frac{1}{2} \gamma g_{m, \text { tail }} R_{p}\right)}{\pi^{2} R_{p}^{3} I_{\text {tail }}^{2} C^{2}(\Delta \omega)^{2}}\right) .
\end{aligned}
$$

\subsubsection{LC-VCO with Noise Filter}

In [1], Hegazi proposed several VCOs with noise filters. According to his study, for current sources, only thermal noise around second harmonics causes phase noise. Hence, the main purpose of adding noise filters is to filter out second harmonics. 


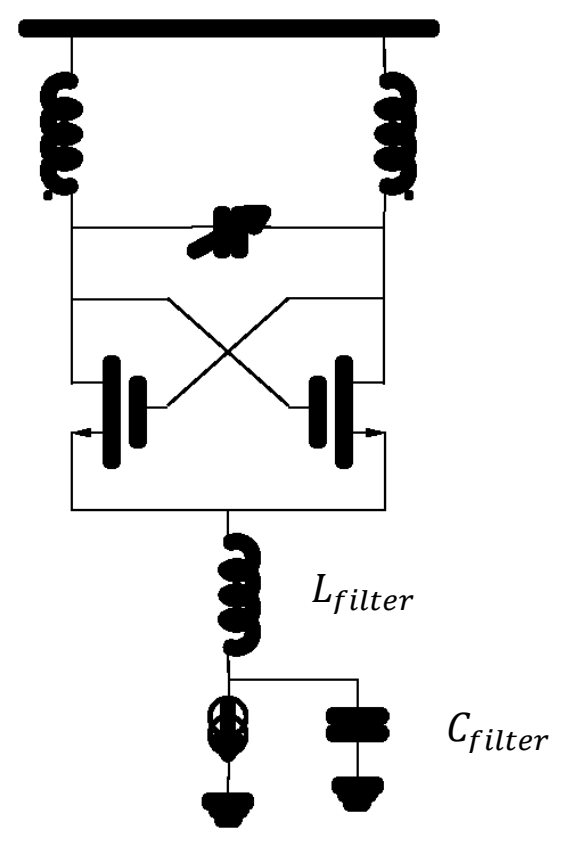

Figure 2.10: LC-VCO with noise filter

no current flowing through the current source. At the same time, even harmonics flow in a common-mode path through the active devices, resonator circuit, and current source.

Because the level of third- and higher-order harmonics in the resonant LC oscillator is negligibly small, the effect of the second harmonic can be taken into account. To prevent the effect of current-source low-noise modulation of the second harmonic, it is critical to provide low impedance for the second harmonic. In other words, it is necessary to create a filter to filter out the second harmonic. Such an approach to phase noise improvement is called a filtering technique. Several filtering techniques have been applied to differential LC-VCOs.

The complete noise filter consists of a tail inductor and a tail capacitor (see Figure 2.10). In a different application, the filter can be implemented with either capacitor only or inductor only.

\subsubsection{LC-VCO Based on Transformer Feedback}




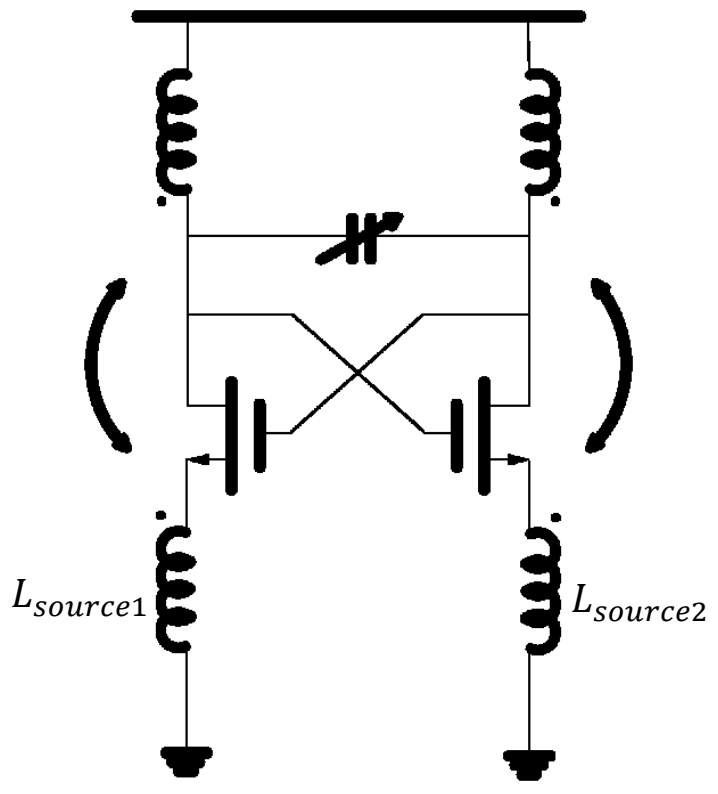

(a)

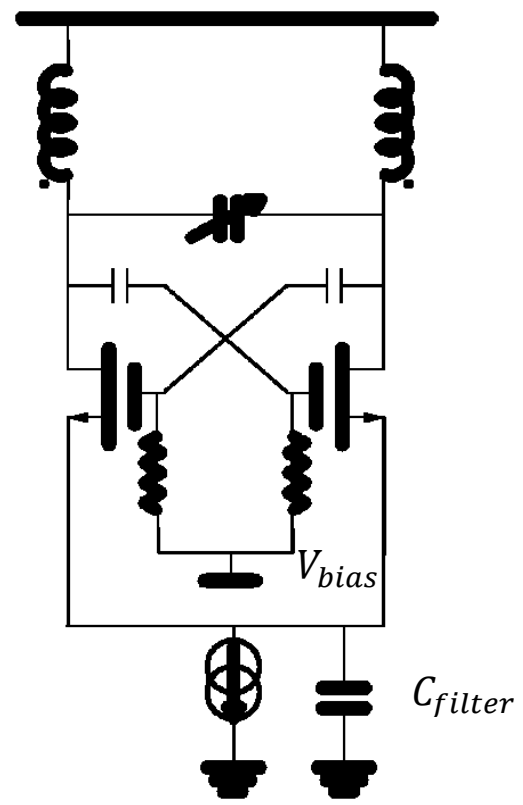

(b)

Figure 2.11: (a) LC-VCO based on transformer feedback. (b) Class-C VCO

In [18], the author proposed a VCO topology based on transformer feedback. Using a transformer can enlarge the voltage headroom, which is highly beneficial under lowvoltage supply conditions. The output voltage swing may also be enlarged, producing lower phase noise.

The circuit can also work like a Colpitts oscillator, namely the conduction angles of the transistors are decreased, which results in the reduction of effective noise. This also contributes to a better phase noise performance.

\subsubsection{Class-C VCO}

Class-C VCO has become popular recently. Many studies have examined class-C VCO in [6 - 21]. Figure 2.11(b) shows one very widely used class-C topology.

The main idea of class-C VCO is to make the transistors work in the class-C mode, namely shape the injected current into tall, narrow pulses. Class-C operation allows for larger current delivery efficiency. Achieving high efficiency in oscillators is 

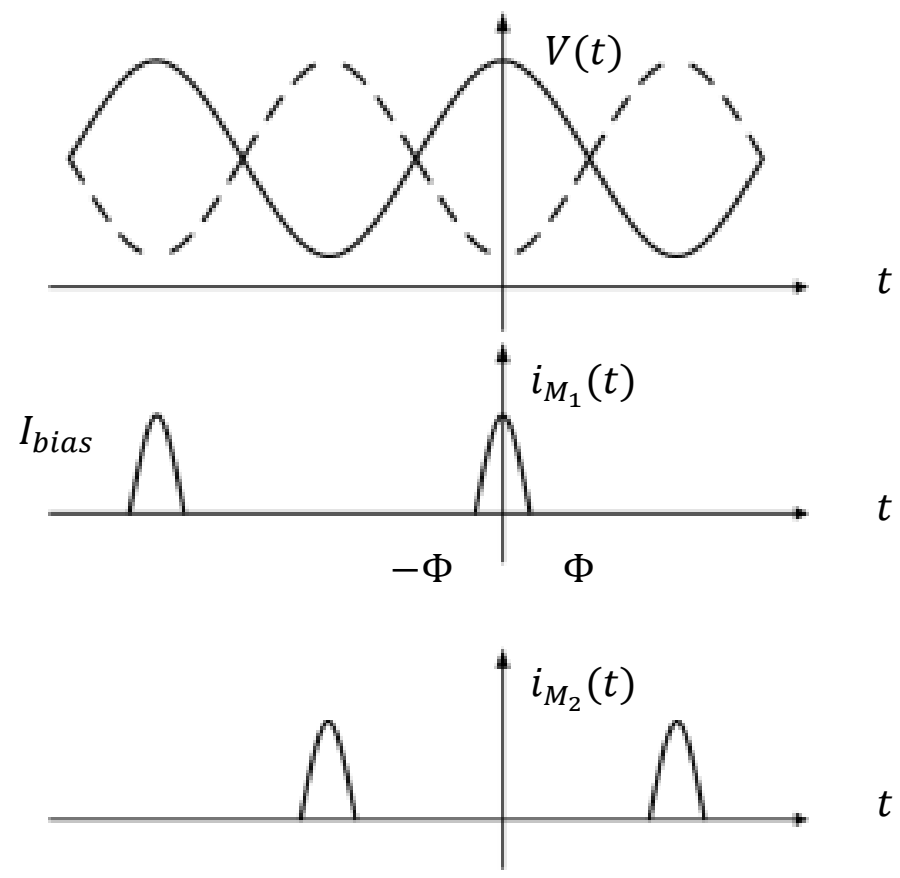

Figure 2.12: Time domain waveforms for class-C VCO.

beneficial for increasing battery life, reducing heat dissipation and enhancing reliability of both measurement equipment and radio transmitters. What's more, it maximizes the oscillation amplitude. At the same level of power consumption, phase noise can be improved by $3.9 \mathrm{~dB}$ theoretically. In other words, to achieve the same phase noise level, more than $50 \%$ of the current can be saved [6].

The time domain waveforms are shown in Figure 2.12. Assume the conduction angles of transistors are $2 \Phi\left(\Phi<\frac{\pi}{2}\right)$, according to [8], the amplitude can be given as

$$
V_{\text {peak }} \approx 2 I_{1} R_{p} \approx 2 I_{\text {tail }} R_{p}
$$

where $\Gamma(\omega t)$ and noise power density of the tank is identical to (29)(30) respectively. The NMF $\alpha(\phi)$ from the switching pair is

$$
\alpha(\phi)=\sqrt{\cos (\phi)-\cos (\Phi)}
$$

Hence ISF for a single end is given by 


$$
\Gamma_{M_{1}, e f f, r m s}^{2}=\frac{1}{2 \pi} \int_{-\Phi}^{\Phi} \frac{1}{4} \sin ^{2}(\phi)(\cos (\phi)-\cos (\Phi)) d \phi .
$$

Using Taylor expansion,

$$
\Gamma_{M_{1}, \mathrm{eff}, \mathrm{rms}}^{2} \approx \frac{1}{8 \pi} \cdot \frac{2}{15} \Phi^{5}\left(1-\frac{11}{42} \Phi^{2}\right) \approx \frac{\Phi^{5}}{60 \pi} .
$$

For a single-ended transistor, the instantaneous transconductance of $M_{1}$ is,

$$
g_{m}(\phi)=\beta V_{\text {peak }}(\cos (\phi)-\cos (\Phi)) .
$$

therefore

$$
I_{\text {tail }}=2 \cdot \frac{1}{2 \pi} \int_{-\Phi}^{\Phi} \frac{\beta V_{\text {peak }}^{2}}{2}(\cos (\phi)-\cos (\Phi))^{2} d \phi \approx \frac{\beta V_{\text {peak }}^{2}}{15 \pi} \Phi^{5} .
$$

After transformation,

$$
\Phi=\left(\frac{15 \pi}{\beta V_{\text {peak }}^{2}} \frac{I_{\text {tail }}}{2}\right)^{1 / 5} .
$$

Substitute (42) into (39), given,

$$
\Gamma_{M_{1}, \mathrm{eff}, \mathrm{rms}}^{2}=\frac{I_{\text {tail }}}{8 \beta V_{\text {peak }}{ }^{2}} .
$$

Hence the total phase noise is, 


$$
\begin{aligned}
\mathcal{L}_{\text {total }}(\Delta \omega)= & 10 \log \left(\frac{\Gamma_{\text {rms,drain,eff }}^{2}}{q_{\text {max }}^{2}} \cdot \frac{\overline{\frac{l_{R_{p}}^{2}}{\Delta f}}}{2 \Delta \omega^{2}}\right) \\
& =10 \log \left(\frac{2 k_{B} T\left(1+\gamma g_{m, M_{1}} R_{p}+\frac{1}{2} \gamma g_{m, \text { tail }} R_{p}\right)}{R_{p}^{3} I_{\text {tail }}^{2} C^{2}(\Delta \omega)^{2}}\right) .
\end{aligned}
$$

\subsubsection{Other Technique and circuits}

In [26], Soltanian proposed a tail current shaping technique. Starting with the mathematical derivation, the author gave a conclusion that narrower tail current pulses lead to less phase noise. Ideally, if the conduction angle of the switching pair approaches 0 , the phase noise can be improved by $9.1 \mathrm{~dB}$.

Hence, to reduce the conduction angle, a peak detector is added to detect the peaks and control the tail current periodically (see Figure 2.14). In fact, this circuit can be viewed as another version of class-C VCO with square shape current pulses.

The amplitude of the output voltage is given by

$$
V_{\text {peak }} \approx \operatorname{sinc}\left(\frac{\Phi}{\pi}\right) I_{\text {tail }} R_{p}
$$

NMF is

$$
\alpha(\varphi)=\frac{\phi}{2 \Phi}
$$

Hence

$$
\Gamma_{M_{1}, e f f, r m s}^{2}=\frac{1}{2 \pi}\left(\Phi-\frac{1}{2} \sin (2 \Phi)\right) .
$$



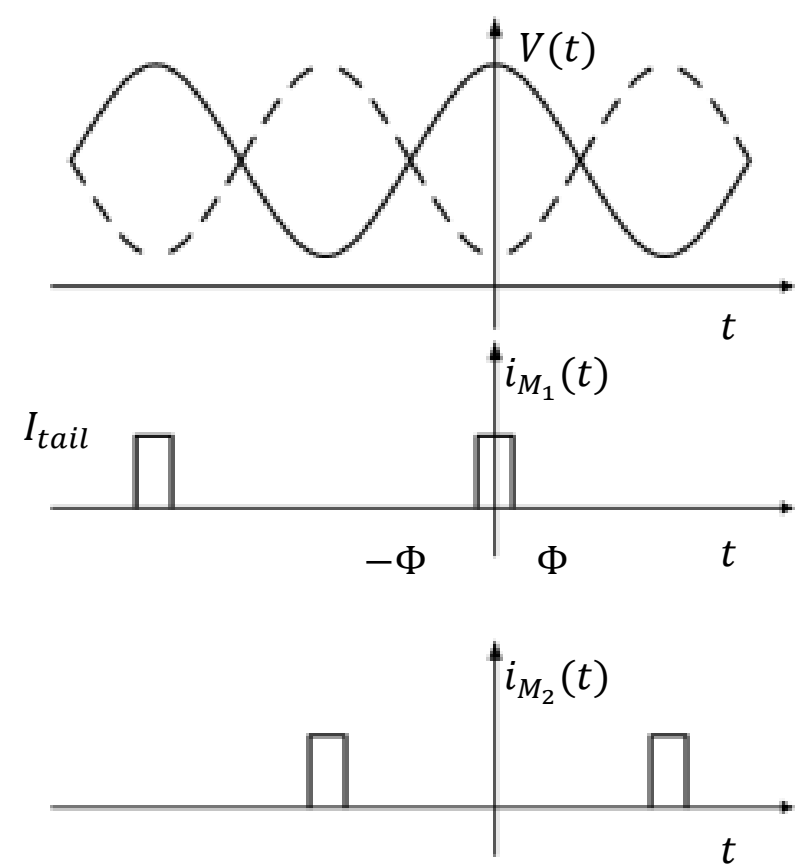

Figure 2.13: Time domain waveforms for VCO using tail current shaping technique.

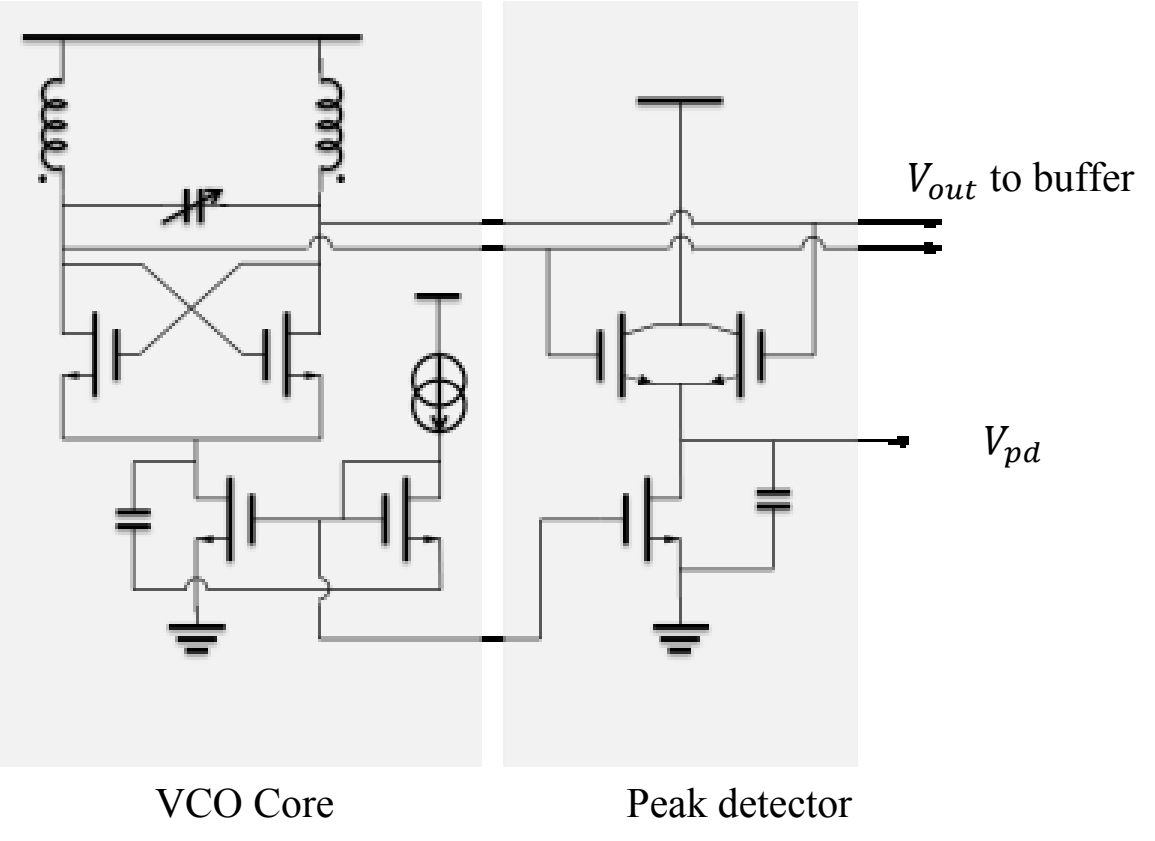

Figure 2.14: Schematic of the fabricated VCO2 core and differential bipolar peak detector. 


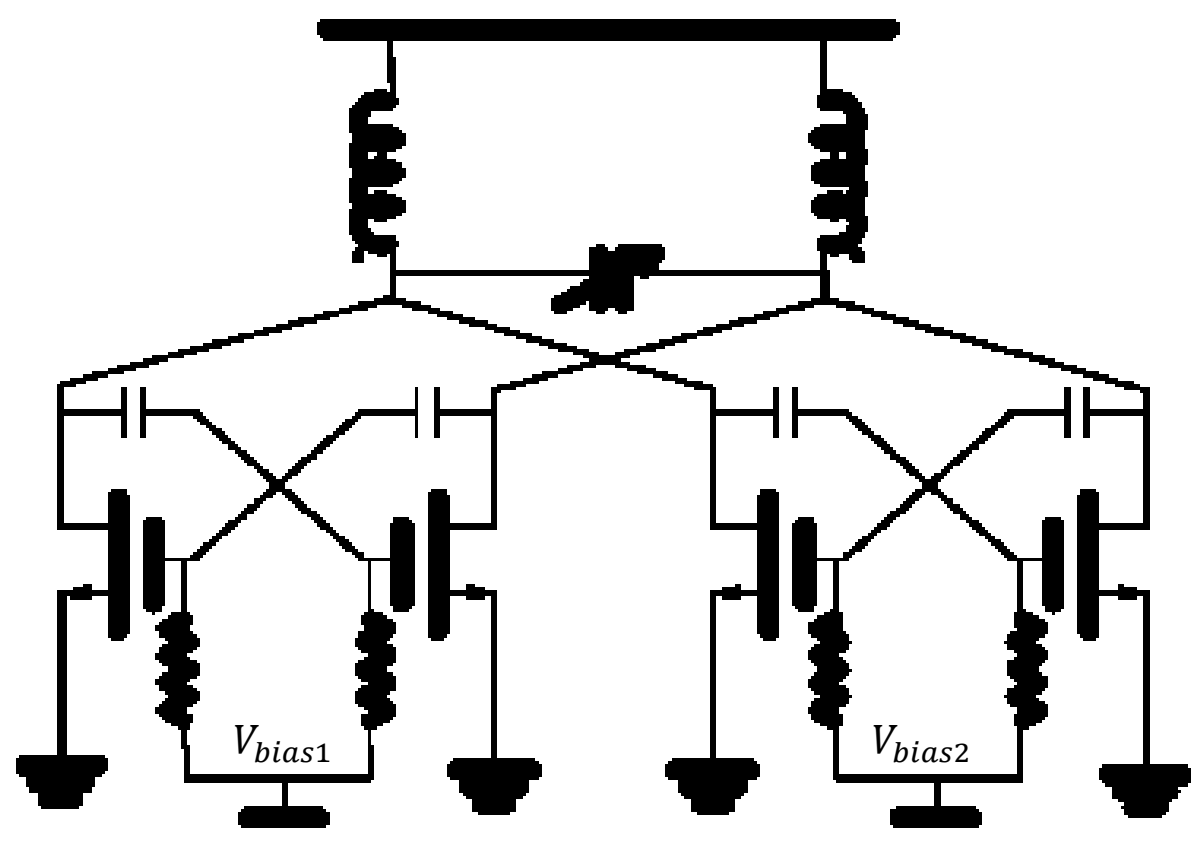

Figure 2.15: Dual-Conduction class-C VCO

Phase noise is therefore given by,

$$
\begin{aligned}
& \mathcal{L}_{\text {total }}(\Delta \omega)=10 \log \left(\frac{\Gamma_{\text {rms,drain, eff }}^{2}}{q_{\text {max }}^{2}} \cdot \frac{\overline{\Delta f}}{2 \Delta \omega^{2}}\right) \\
& =10 \log \left(\frac{4 k_{B}^{2} T\left(1+\gamma g_{m, M_{1}} \frac{\Phi-\frac{1}{2} \sin (2 \Phi)}{\sqrt{2 \pi \Phi}}+\gamma g_{m, \text { tail }} R_{p} \frac{\Phi-\frac{1}{2} \sin (2 \Phi)}{2 \Phi}\right)}{\pi^{2} R_{p}^{3} I_{\text {tail }}^{2} C^{2}(\Delta \omega)^{2}}\right) .
\end{aligned}
$$

Another interesting circuit is proposed by Okada in [23]. The circuit is designed with super low voltage supply of $0.2 \mathrm{~V}$. The circuit employed dual class-C operation. One branch is designed with comparatively large conduction angle to ensure robust startup, while the other branch is designed with smaller conduction angle to reduce effective 
noise (see Figure 2.15). The circuit achieved ultra-low power consumption; however the trade-off is a less favorable phase noise performance.

\subsection{Conclusion}

This chapter reviews the VCO theory, including linear and non-linear approaches as well as circuit examples from literatures It turned out although many attempts have been made to predict the phase noise performance and some of them have achieved quite precise prediction, the existing circuits showed weak connections to these theory, namely those theory, are not insightful enough to guide a practical VCO design. Hence, finding insight for low noise VCO designs becomes the first target of this thesis. 


\section{Chapter: Proposed VCO Topology}

\subsection{Introduction}

This chapter first presents a study on phase noise generation. The study begins by examining the relation between phase noise and harmonics. However, simulation results show that harmonics are not the only factor to determine the phase noise. After studying the mechanism of the generation of phase noise, it is found that the phase noise is determined by the transfer function and harmonic components of the circuit. Hence a VCO design guideline has been concluded to guide VCO design. Based on this research, a novel VCO topology is proposed. Experiments are carried out on the proposed circuit with two other reference topologies to verify the circuit's ability to reduce the transfer function and harmonics, which yields better phase noise performance.

\subsection{Study on Relation between Phase Noise and Harmonics}

According to the energy definition of the quality factor, in an ideal lossless tank,

$$
Q=\omega \frac{E_{\text {stored }}}{E_{\text {diss }}} \rightarrow \infty .
$$

given

$$
\Delta \omega_{3 d B}=\frac{\omega_{c}}{Q} \rightarrow 0
$$

In reality, however, $Q$ of a tank is not infinite. Phase noise and harmonic components are thus unavoidable. Therefore it is reasonable to assume that harmonics cause or partially cause the phase noise. 
Researchers have studied the relation between phase noise and harmonics [3335]. In 1933, Groszkowski mathematically proved that the presence of harmonics in the tank current of a LC-VCO, which induced by the nonlinearity of the circuit, results in a shift of the oscillation frequency from the tank resonance frequency in [35]. This shift is shown in,

$$
\frac{\Delta \omega}{\omega_{0}}=-\frac{1}{2 Q^{2}} \sum_{n=2}^{\infty} a_{k} n_{k}^{2}
$$

where $a_{k}$ being a coefficient depends on the topology and

$$
n_{k}=\frac{I_{k}}{I_{1}}
$$

where $I_{k}$ is the amplitude of the $\mathrm{k}^{\text {th }}$ harmonic of the tank current. If any noise changes $n_{k}$, a spurious frequency modulation will arise and then low frequency noise will be upconverted into phase noise sidebands [36]. Also, in [1], the authors demonstrated that by adding filters on VCOs, second harmonics will be filtered out, and phase noise is consequently decreased. While these researchers all discussed the relation between phase noise and harmonics, no study has reportedly examined the relationship.

Therefore, in this thesis, the research begins with examining the relationship. A VCO with a filter (see Figure 3.1) has been employed to research the relationship. The filter can be adjusted by tuning the inductor, which yields different harmonic components.

After many experiments, it was found that the third harmonic is of most importance. This matches the results demonstrated in [33]. Even order harmonics are absent because of the symmetry of the circuit. Other harmonics that are higher than the third harmonics are relatively small and can be ignored. This is because the filtering 


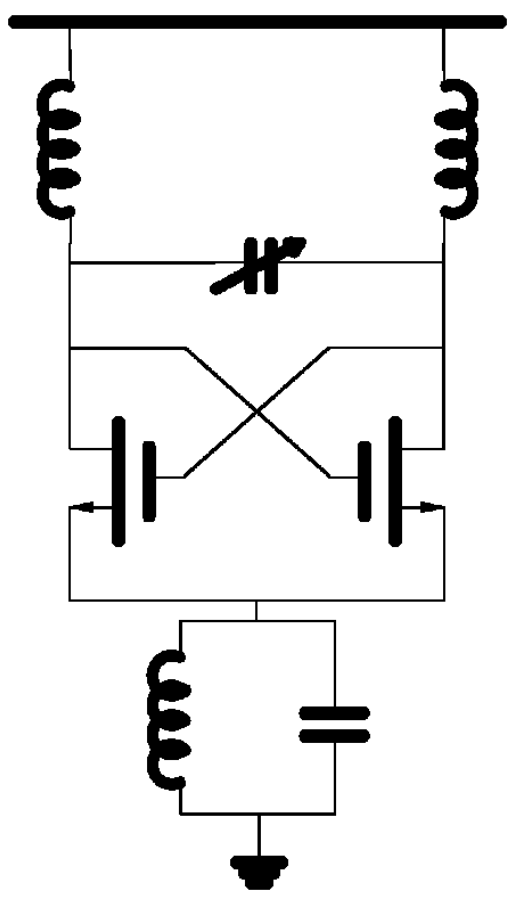

Figure 3.1: VCO with tunable filter.

effect of the tank circuit. Therefore, the Total Harmonic Distortion (THD) can be approximated by,

$$
\mathrm{THD} \cong \frac{V_{3 r d}}{V_{1 s t}}
$$

where $V_{3 r d}$ is the voltage amplitude of third harmonics in the differential output signal, and $V_{1 s t}$ is the voltage amplitude of the fundamental component. Using $L_{\text {tank }}=$ $2 n H, C_{\text {tank }}=2 p F$, Figure 3.2- Figure 3.4 shows the phase noise performance with various inductances of the filters.

From the simulation results, it can be observed that the predicted trend is confirmed, namely higher THD has the tendency to produce more phase noise. However, these results indicate that the phase noise performance may not only depend on harmonics. 


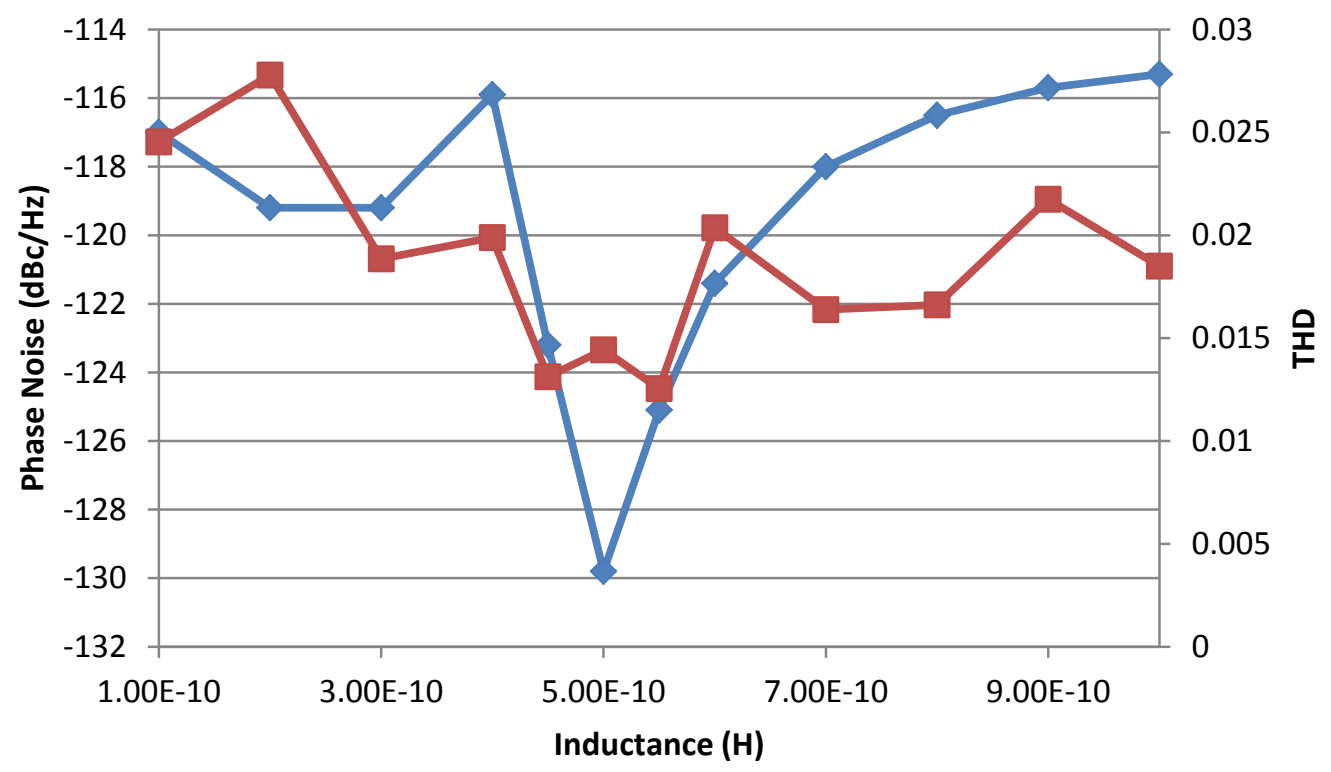

$\longrightarrow$ Phase Noise $\quad$ THD

Figure 3.2: Simulation results for the phase noise performance with different harmonics when $\mathrm{C}=2 \mathbf{p F}$.

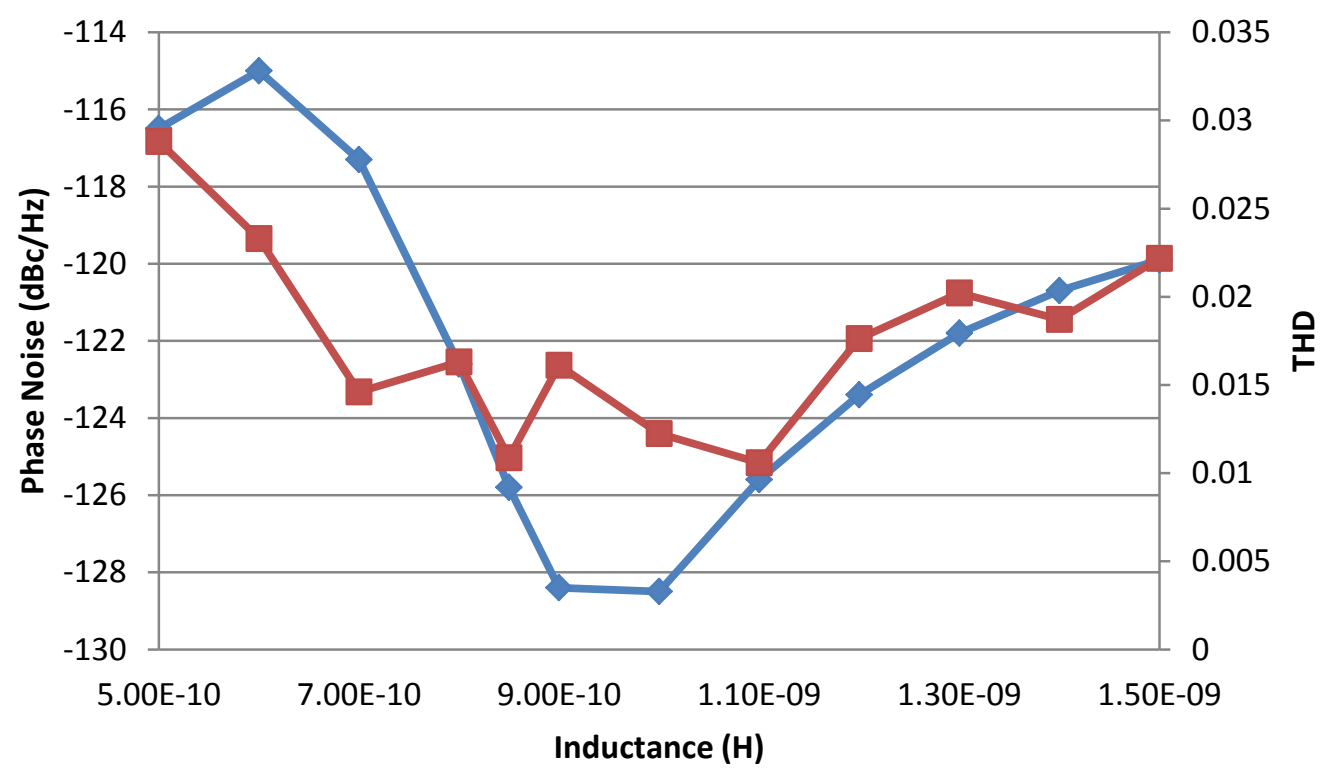

$\leadsto$ Phase Noise $\quad-$ THD

Figure 3.3: Simulation results for the phase noise performance with different harmonics when

$$
\mathrm{C}=\mathbf{1 p F} \text {. }
$$




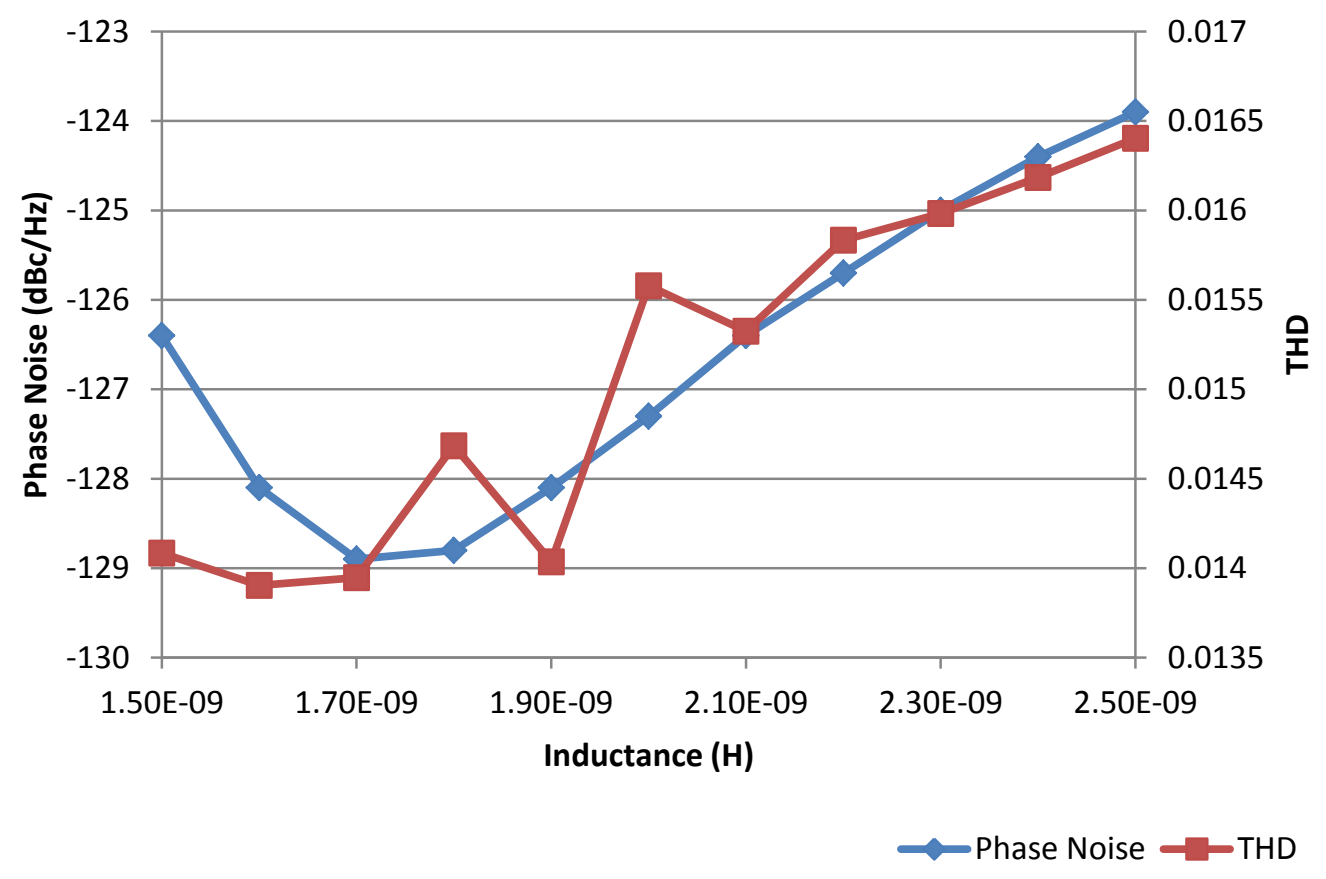

Figure 3.4: Simulation results for the phase noise performance with different harmonics when

$$
\mathrm{C}=0.5 \mathrm{pF}
$$

\subsection{Generation of Phase Noise}

To discover the other factor that determines the phase noise, the mechanism of the generation of phase noise is explored first.

Here only white noise is analyzed. Flicker noise is ignored because low frequency noise will be filtered out by the PLL loop in VCO implementations. Hence, the noise power spectral density of the noise source should be nearly constant throughout the frequency spectrum ideally. According to the Nyquist theorem, the noise spectrum $S_{v}(\omega)$ of the circuit is related to the real part of the impedance. The noise power spectrum is therefore modified by the circuit by

$$
S_{v}(\omega)=2 k T \cdot \operatorname{ReZ}(j \omega)
$$

To observe a general case, the circuit can be simplified by connecting an independent current source $n_{i}(t)$ (noise), a noiseless resistor $R$ and a network containing 


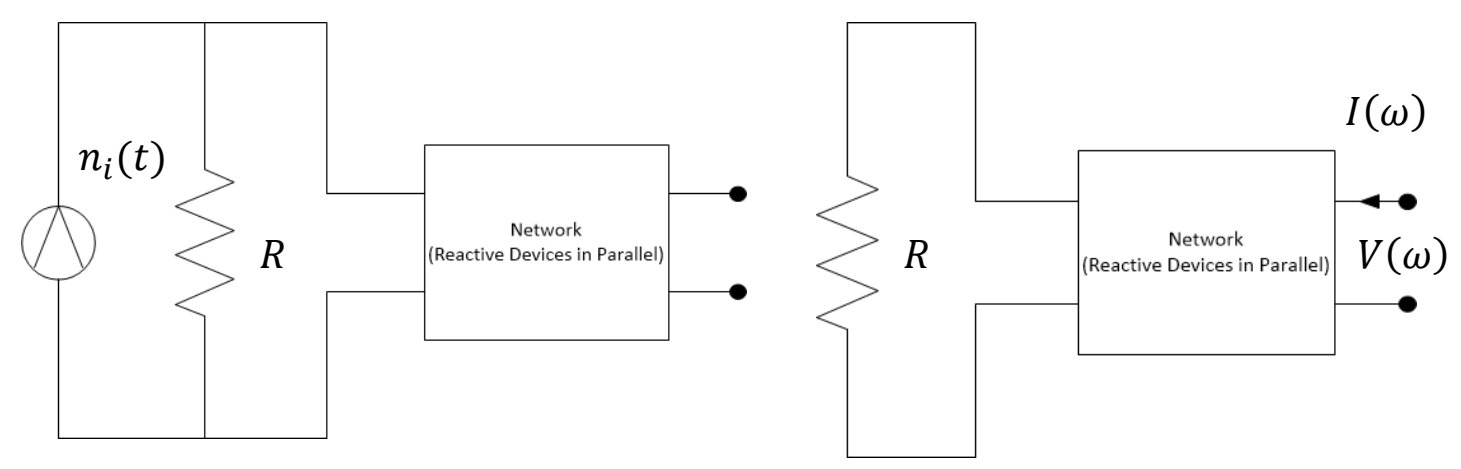

Figure 3.5: Black box model for oscillators [37].

only reactive devices in parallel (see Figure 3.5). Thus, the system transfer function $H(\omega)$ is equal to

$$
H(\omega)=\frac{V(\omega)}{I(\omega)}
$$

where $V(\omega)$ and $I(\omega)$ represent the amplitude of the voltage across the resistor and $I(\omega)$ represents the amplitude of the input sine wave current. Because the circuit is assumed to be noiseless, the input power is equal to the output power, given

$$
|I(\omega)|^{2} \cdot \operatorname{ReZ}(j \omega)=\frac{|V(\omega)|^{2}}{R} .
$$

By substituting equation (55) with quation (56), we obtain

$$
\begin{gathered}
S_{v}(\omega)=S_{n_{i}}(\omega) \cdot|H(\omega)|^{2}, \\
S_{n_{i}}(\omega)=\frac{2 k T}{R} .
\end{gathered}
$$

Note that equations (3.4)-(3.8) are cited from [37]. 


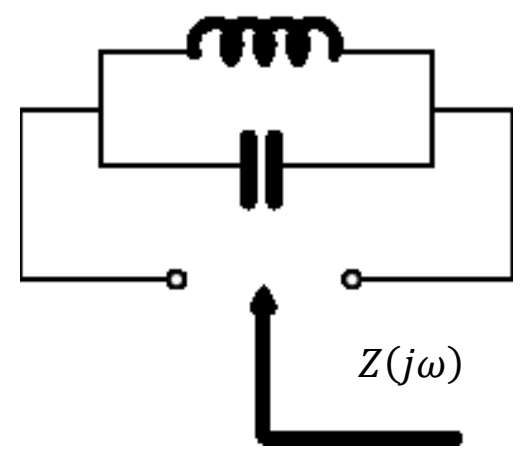

Figure 3.6: LC resonator.

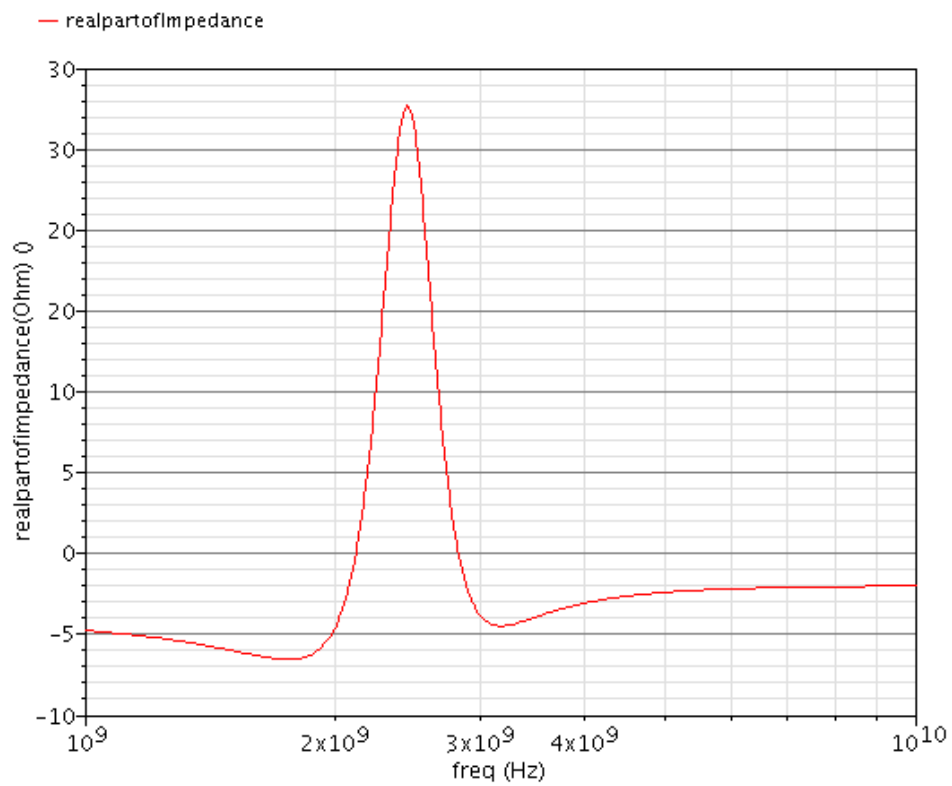

Figure 3.7: Impedance of a LC-VCO (example).

In LC-tank VCOs, the network in Figure 3.5 should be a LC tank (see Figure 3.6). In an ideal LC circuit, at resonant frequency $f_{c}$, where

$$
f_{c}=\frac{1}{2 \pi \sqrt{L C}},
$$




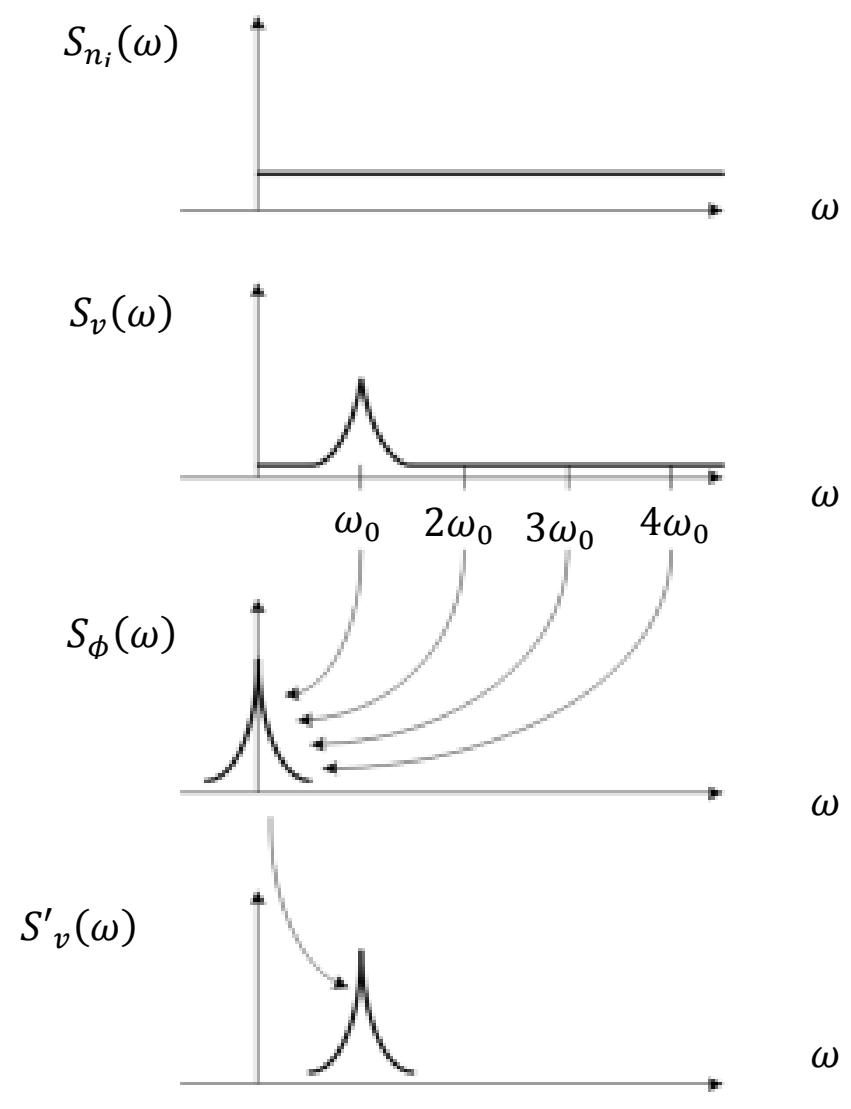

Figure 3.8: Conversion of noise to phase noise

and the real part of the impedance looking into the tank (see Figure 3.6), $\operatorname{Re} Z(j \omega)$, approaches infinity since

$$
Z(j \omega)=\frac{1}{\frac{1}{Z_{c}}+\frac{1}{Z_{L}}}=\frac{1}{\frac{1}{j \omega L}+j \omega C}=\frac{1}{0} \rightarrow \infty .
$$

In reality, due to the existence of parasitic resistance, the tank cannot be simplified into a $\mathrm{LC}$ resonator. The impedance at the resonant frequency is finite, and the shape of the impedance is pulse-like. Figure 3.7 gives an example of the impedance of a LC-VCO. According to the equation (54), the noise power spectral density should share the same shape as that of the impedance. 
However, because of the nonlinearity, there are always harmonic components existing in an oscillator. These harmonics will, in turn, mix with the noise. Recall the frequency mixing equations between the two signals $A_{1} \cos (\omega t)$ and $A_{2} \cos (v t)$,

$$
v_{\text {out }}=A_{1} \cos (\omega t) \cdot A_{2} \cos (v t)=\frac{A_{1} A_{2}}{2}[\cos ((\omega+v) t)+\cos ((\omega-v) t)]
$$

The sum and difference frequencies will be produced when these two signals are mixed. In this case, noise around the integer multiples of the oscillating frequency is downconverted into low frequency noise sidebands by the harmonics. Namely, the energy around the harmonics is taken down by the harmonics, accumulating around dc. Then noise around DC will then be up-converted by the fundamental frequency, forming the phase noise sidebands around the carrier. Phase noise is then generated. The whole process is illustrated in Figure 3.8.

\subsection{LC-VCO Design Guideline}

Studies of the generation of phase noise benefit designing low phase noise VCOs. In previous studies, some LC-VCO design guidelines for low phase noise VCOs can be achieved through those VCO models. For example, from Leeson's empirical equation (10), it can be concluded that one should maximize the $\mathrm{Q}$ factor and maximize the power of the output signal to minimize phase noise.

From the mixer model mentioned in Chapter 2.2.4 (see equation (19)), it can be concluded that regardless of what topology is used to reduce phase noise, the voltage amplitude of the output signal should be maximized, while the noise factor should be minimized. The noise factor is dependent on the dimension of the active devices.

However, the voltage amplitude of the output signal and the noise factor are not always orthogonal. Usually, larger amplitude is achieved by enlarging $g_{m}$ of the active devices, but enlarging $g_{m}$ will in turn change the noise factor. Whether it increases the noise factor depends on real case calculations. 


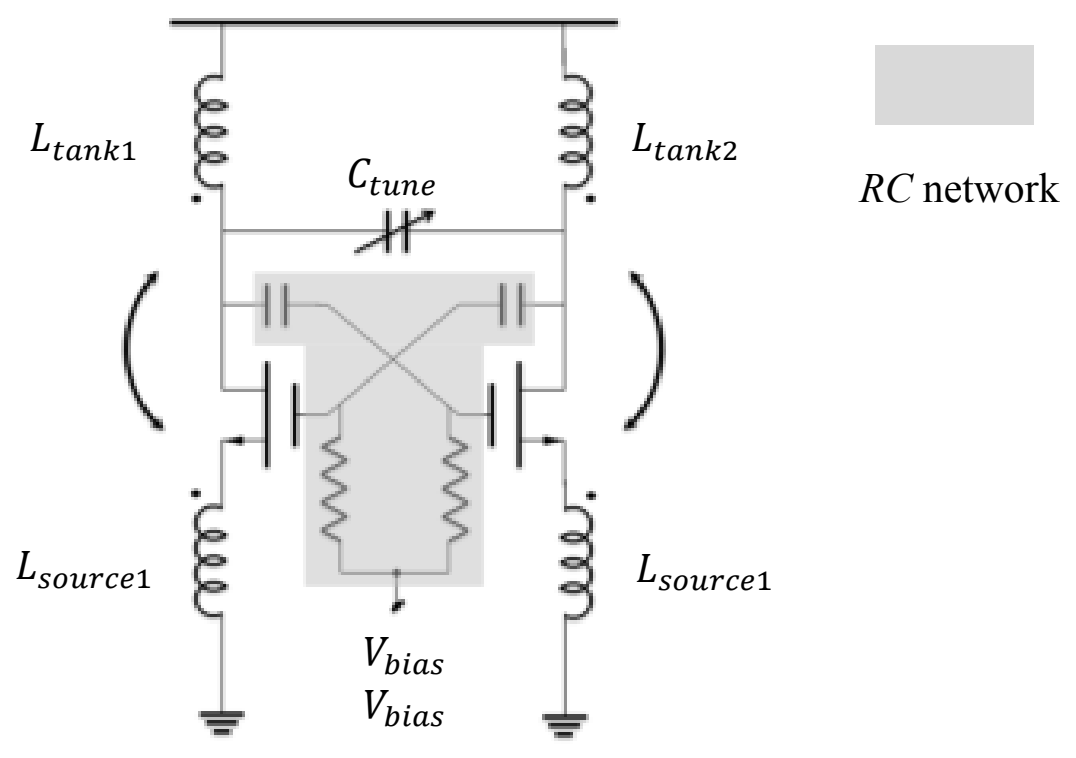

Figure 3.9: Schematic for proposed VCO

From Hajimiri's ISF theory (see equation (21)), it can be observed that one way to reduce the phase noise is to increase the maximal charge across the tank. This method can be achieved by enlarging the voltage amplitude because the charge is proportional to the voltage amplitude. Another away to reduce phase noise is by decreasing the $\Gamma_{r m s}^{2}$. However, decreasing $\Gamma_{r m s}^{2}$ is not intuitive, which makes the theory less practical in guiding VCO design [6].

In this thesis, the analysis in the previous chapter reveals the link between the phase noise and nonlinearity of the circuit, which is, the phase noise performance of an oscillator is determined by the presence of noise and harmonic components of the circuit. This conclusion serves to guide the design of oscillators with low phase noise.

First, regardless of the harmonics, when the noise source remains the same, the lesser the noise amplitude is, the better the phase noise performance is. According to (57), noise spectral density is proportional to the square of the noise to output transfer function. Therefore, if the magnitude of the transfer function is reduced or narrower bandwidth, the presence of noise of the oscillators can be proven.

Second, regardless of the presence of noise, if one can enlarge the fundamental component and suppress higher harmonics, less noise would be converted around the carrier. The phase noise performance is in turn improved. 


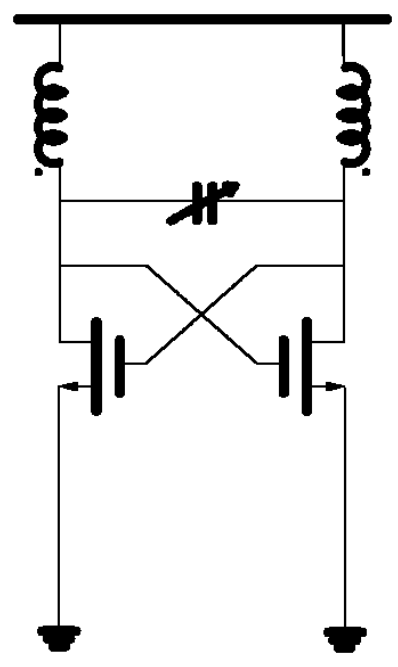

(a)

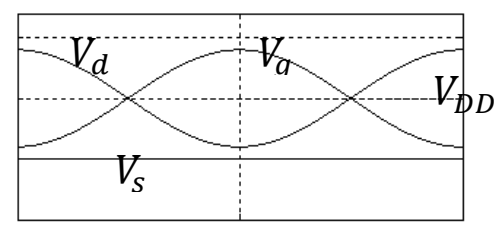

(d)

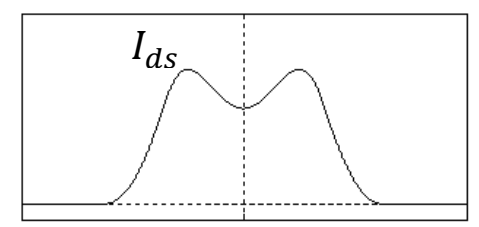

(g)

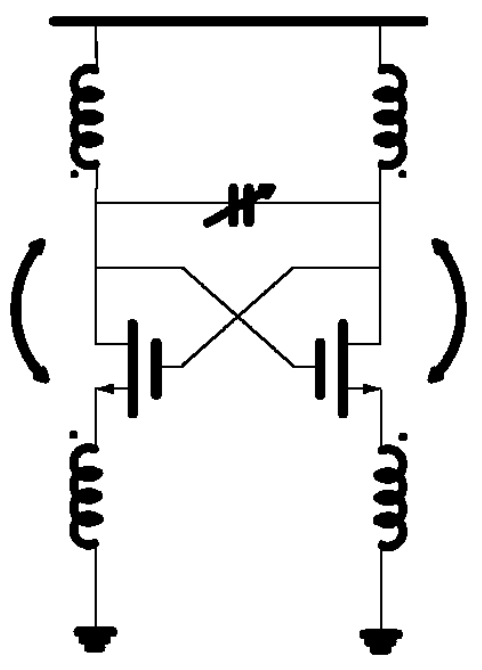

(b)

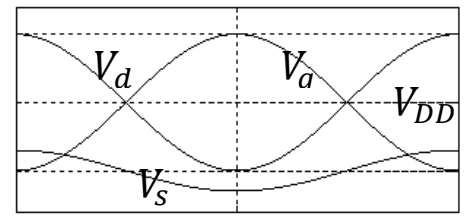

(e)

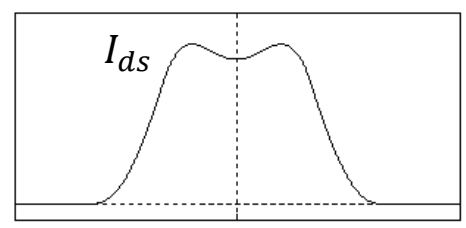

(h)

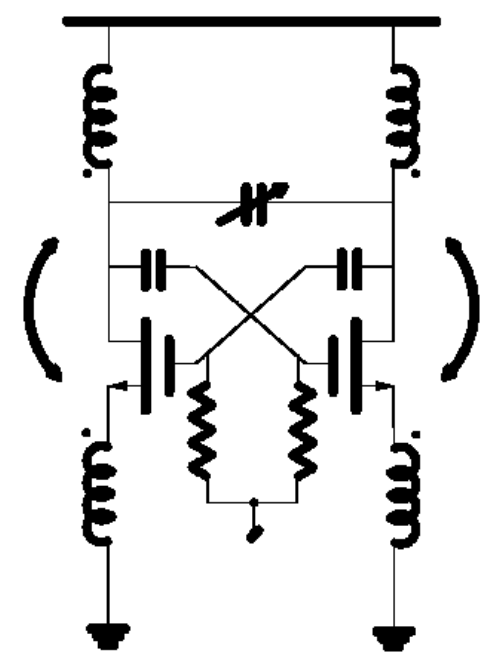

(c)

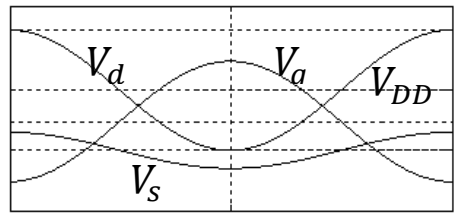

(f)

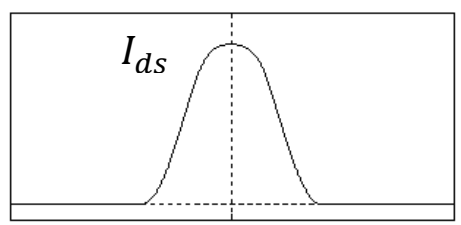

(i)

Figure 3.10: Topology for (a) VCO1 (b) VCO2 (c) VCO3 (Proposed). (d)(e)(f) show voltage waveform in time domain for three VCOs. (g)(h)(i) show injected current in time domain.

Overall, better phase noise performance can be achieved by reducing either the presence of noise or the harmonic components, or both. It should be noted that the such design guideline is concluded under the condition that flicker noise is ignored.

\subsection{Proposed VCO Topology}

Following the conclusion in the previous chapter, it can be easily concluded that an ideal candidate VCO circuit should minimize both transfer function and harmonics. Among the circuits that have been reviewed in Chapter 2, VCOs with noise filter and transformer feedback VCOs made efforts to reduce the harmonics - the former tried to filter out the second harmonics, while the latter provided more voltage headroom, which in turn 
reduced the nonlinearity. However, no effort has been made to modify the transfer function directly.

Therefore, a new VCO topology has been proposed to achieve better phase noise performance (see Figure 3.9). The circuit can be interpreted as adding transformers and an $R C$ feedback network onto the simplest LC-tank VCO (see Figure 3.10(a)). The $R C$ network is highlighted in the figure.

As with the transformer feedback $\mathrm{VCO}$, the aim of adding transformers is primarily to reduce the harmonics. By coupling inductors $L_{\text {source } 1}$ and $L_{\text {source } 2}$ to tank inductors $L_{\text {tank1 } 1}$ and $L_{\text {tank2 }}$, respectively, in time domain, voltage at the source node of the transistors $V_{S}$ becomes variant instead of remaining at $0 \mathrm{~V}$ in the simplest VCO topology (see Figure 3.10(a)). This provides two advantages. First, it provides more voltage headroom, which enlarges the fundamental component in the output signal. Second, through careful design, the current injected into the tank can be shaped closer to sinusoidal (see Figure 3.10 (i)). Using the negative resistance model (see Figure 2.2), the shape of the voltage across the tank will be closer to sinusoidal, producing fewer harmonics.

The main purpose of adding the $R C$ feedback network is to modify the transfer function. This method can be considered as adding $R C$ filters in the feedback network. By setting appropriate $V_{\text {bias }}$, the feedback gain can be reduced. The bandwidth of transfer function will become much smaller.

\subsection{Simulation}

Here, three VCOs are compared. Topologies are shown as above [Figure 3.10 (a)-(c)]. VCO1 is the simplest VCO topology, VCO2 employs transformers only (without the RC feedback network), and VCO3 is the proposed topology. The same tank is employed in three circuits to ensure similar quality factors. Supply voltage is also kept identical. Transformers in VCO2 and VCO3 are the same, which also presents the same coupling factors to the tank inductors.

To explore how much the proposed circuit may benefit the phase noise performance, two experiments were carried out. Experiment I is designed to show how 
Table 2: Parameters for Experiment I

\begin{tabular}{c|ccc}
\hline & VCO1 & VCO2 & VCO3 \\
\hline Supply Voltage (V) & 0.5 & 0.5 & 0.5 \\
$\boldsymbol{L}_{\text {tank1,2 }}(\mathbf{n H})$ & 2 & 2 & 2 \\
$\boldsymbol{C}_{\text {tank1,2 }}(\mathbf{p F})$ & 2 & 2 & 2 \\
$\boldsymbol{R}_{\text {tank1,2}}(\mathbf{o h m})$ & 2 & 2 & 2 \\
$\mathbf{W} / \mathbf{L}$ & $26 \mathrm{um} / 120 \mathrm{~nm}$ & $26 \mathrm{um} / 120 \mathrm{~nm}$ & $26 \mathrm{um} / 120 \mathrm{~nm}$ \\
$\boldsymbol{L}_{\text {source1,2 }}(\mathbf{n H})$ & 0.6 & 0.6 & 0.6 \\
$\boldsymbol{R}_{\text {source1,2 }}(\mathbf{o h m})$ & 0.6 & 0.6 & 0.6 \\
Coupling Coefficient & - & 0.4 & 0.4 \\
$\boldsymbol{R}_{\text {filter1,2 }}(\mathbf{M o h m})$ & - & - & 100 \\
$\boldsymbol{C}_{\boldsymbol{f i l t e r 1 , 2}}(\mathbf{p F})$ & - & - & 10 \\
\hline
\end{tabular}

Table 3: Parameters for Experiment II

\begin{tabular}{|c|c|c|c|}
\hline & VCO1 & $\mathrm{VCO} 2$ & VCO3 \\
\hline Supply Voltage (V) & 0.5 & 0.5 & 0.5 \\
\hline$L_{t a n k 1,2}(\mathrm{nH})$ & 2 & 2 & 2 \\
\hline$C_{t a n k 1,2}(\mathrm{pF})$ & 2 & 2 & 2 \\
\hline$R_{\text {tank1,2 }}(\mathrm{ohm})$ & 2 & 2 & 2 \\
\hline $\mathbf{W} / \mathbf{L}$ & $22 \mathrm{um} / 120 \mathrm{~nm}$ & $12 \mathrm{um} / 120 \mathrm{~nm}$ & $26 \mathrm{um} / 120 \mathrm{~nm}$ \\
\hline$L_{\text {source } 1,2}(\mathrm{nH})$ & 0.6 & 0.6 & 0.6 \\
\hline$R_{\text {source } 1,2}(\mathrm{ohm})$ & 0.6 & 0.6 & 0.6 \\
\hline Coupling Coefficient & - & 0.4 & 0.4 \\
\hline$R_{\text {filter } 1,2}($ Mohm $)$ & - & - & 100 \\
\hline$C_{\text {filter } 1,2}(\mathrm{pF})$ & - & - & 10 \\
\hline
\end{tabular}

this topology would influence the transfer function and harmonics when the noise sources are the same. To keep the noise source the same, identically sized active devices are 
employed in the three circuits. However, this would make the output amplitude of the three circuits different from each other. Therefore, another experiment, Experiment II, is designed to show how this topology would influence the transfer function and harmonics when the output amplitude is kept the same for the three circuits. Active devices in the three circuits are adjusted to give the same output amplitude, which is $2.0 \mathrm{~V}$ for peak-topeak voltage of the differential voltage output. Parameters for Experiment I and Experiment II are shown in Table 2, respectively.

As aforementioned, the main goal of adding transformers is to reduce the harmonics. Therefore, the harmonics difference in $\mathrm{VCO} 1$ and $\mathrm{VCO} 2$ are mainly compared, as the transformer is the only difference between the two topologies. A large improvement in suppressing harmonics should be obtained in $\mathrm{VCO} 2$ as compared with $\mathrm{VCO} 1$ in both experiments. For transfer function, results for $\mathrm{VCO} 2$ and $\mathrm{VCO} 3$ and mainly focused on how the $R C$ feedback network benefits. A transfer function with much narrower bandwidth and lower peak amplitude should be obtained in VCO3 when compared with $\mathrm{VCO} 2$.

In both experiments, harmonics are obtained first, using the Fast Fourier Transformation (FFT) analysis. For transfer function, instead of using the traditional method, which breaks the loop and calculates the open loop transfer function directly, the Middlebrook method is used to obtain a more accurate result. It is important to note that before this thesis, no study has reported utilizing the Middlebrook method on differential VCOs. Details about the advantages of this method and its implementation are introduced in Appendix A. Here, only the results are presented.

It should be noted that the Middlebrook method can only simulate white noise spectrum. To exclude the influence from flicker noise, flicker noise is turned off first in the simulator to explore how harmonics and transfer function affect phase noise performance from Chapter 3.6.1 to 3.6.3. In Chapter 3.6.4, flicker noise is turned on again in Spectre to see the phase noise performance in the proposed circuit when flicker noise is taken into account.

\subsubsection{Harmonics}


Table 4: Simulation results for Harmonics for Experiment I

\begin{tabular}{l|cccc}
\hline & Fundamental (V) & $\mathbf{2}^{\text {nd }} \operatorname{Harmonics}(\mathbf{V})$ & $\mathbf{3}^{\text {rd }}$ Harmonics $(\mathbf{V})$ & THD \\
\hline VCO1 & 0.938 & - & 0.02724 & 0.0290 \\
VCO2 & 0.847 & - & 0.01802 & 0.0213 \\
VCO3 & 1.054 & 0.0091 & 0.00722 & 0.0110 \\
\hline
\end{tabular}

Table 5: Simulation results for Harmonics for Experiment II

\begin{tabular}{l|cccc}
\hline & Fundamental (V) & $\mathbf{2}^{\text {nd }}$ Harmonics(V) & $\mathbf{3}^{\text {rd }}$ Harmonics (V) & THD \\
\hline VCO1 & 0.8415 & - & 0.01692 & 0.0201 \\
VCO2 & 0.9226 & - & 0.00583 & 0.0063 \\
VCO3 & 1.054 & 0.0091 & 0.00722 & 0.0110 \\
\hline
\end{tabular}

Harmonics from the differential output of the three topologies in Experiment I and Experiment II are shown in Figure 3.11 and Figure 3.12, respectively. The conclusion of the harmonics components are shown in Table 4 and Table 5. Note that the fundamental frequencies of the three circuits change slightly, as VCO2 and VCO3 introduce extra components (e.g. inductors), resulting in shifts in center frequencies. Furthermore, voltage harmonics under $10^{-4} \mathrm{~V}$ are ignored. Therefore, harmonics that are higher than fourth harmonics are ignored.

In Experiment I, third harmonics are dominant in VCO1 and VCO2. Although $\mathrm{VCO} 2$ did not suppress the absolute value of the third harmonics; it efficiently enlarges the fundamental component. The value of THD is reduced by $26.5 \%$. This shows that the transformer suppresses the harmonics. In VCO3, despite the fact that second harmonics are introduced, it still successfully suppressed the third harmonics and increased the fundamental component at the same time. The THD value is slightly higher than it is in $\mathrm{VCO} 2$. Because the only difference between $\mathrm{VCO} 2$ and $\mathrm{VCO} 3$ is the $R C$ feedback network, it can be conjured that the harmonic variation in $\mathrm{VCO} 2$ and $\mathrm{VCO} 3$ is caused by the $R C$ feedback network; however, the variation is very small. 
Frequency

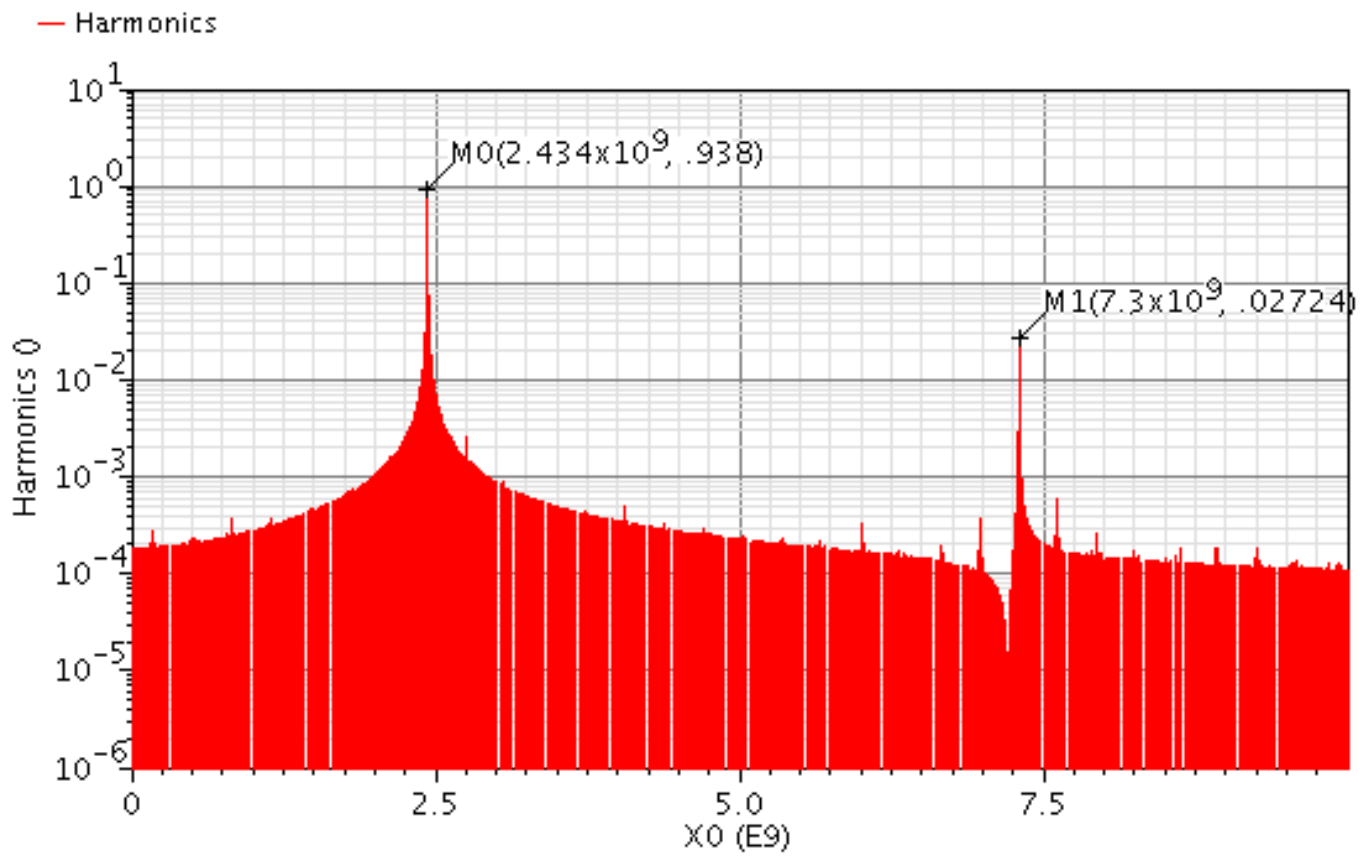

(a)

Expressions

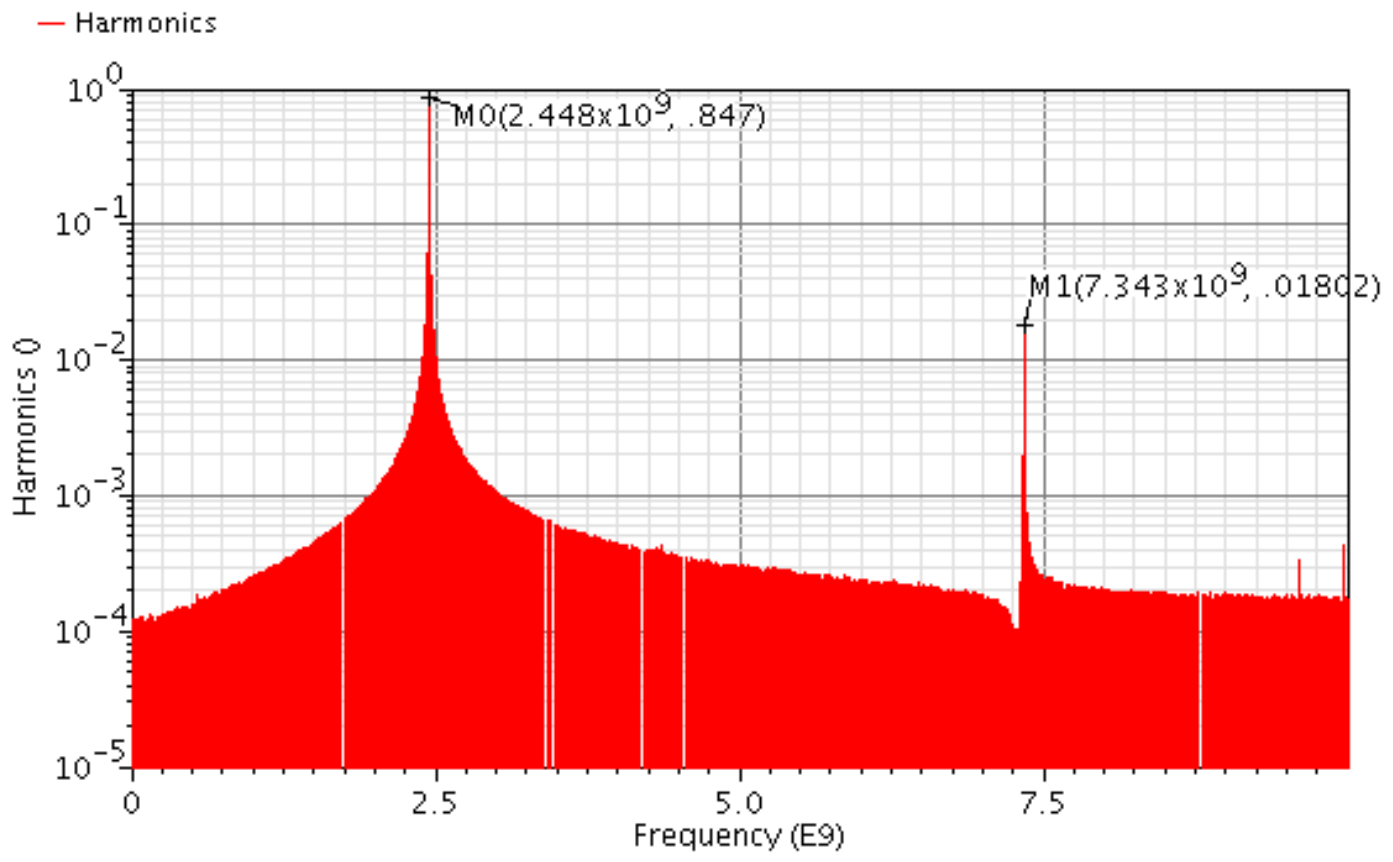

(b) 
Harmonics

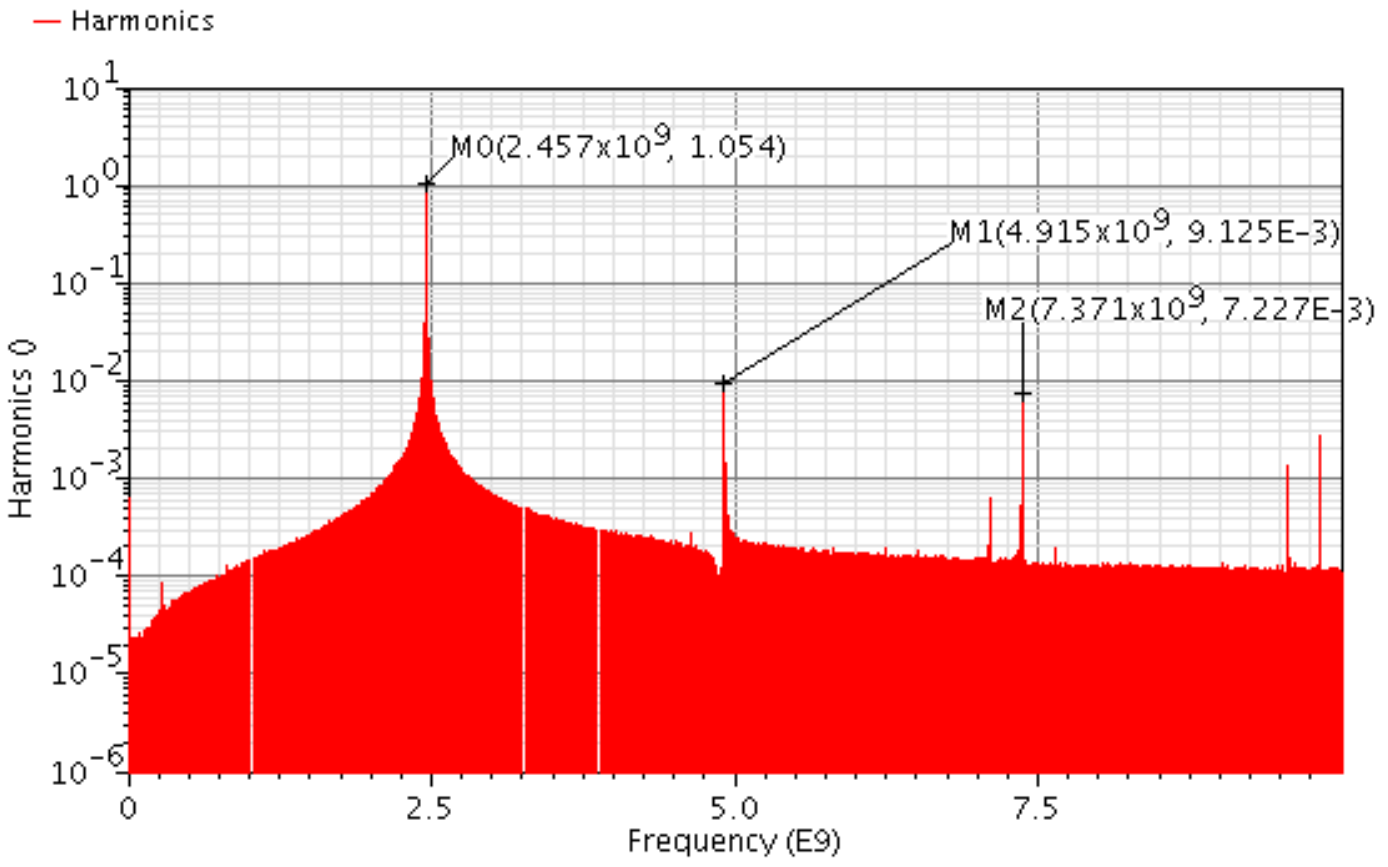

(c)

Figure 3.11 Harmonic simulation of (a) VCO1 (b) VCO2 (c) VCO3 for Experiment I. Expressions

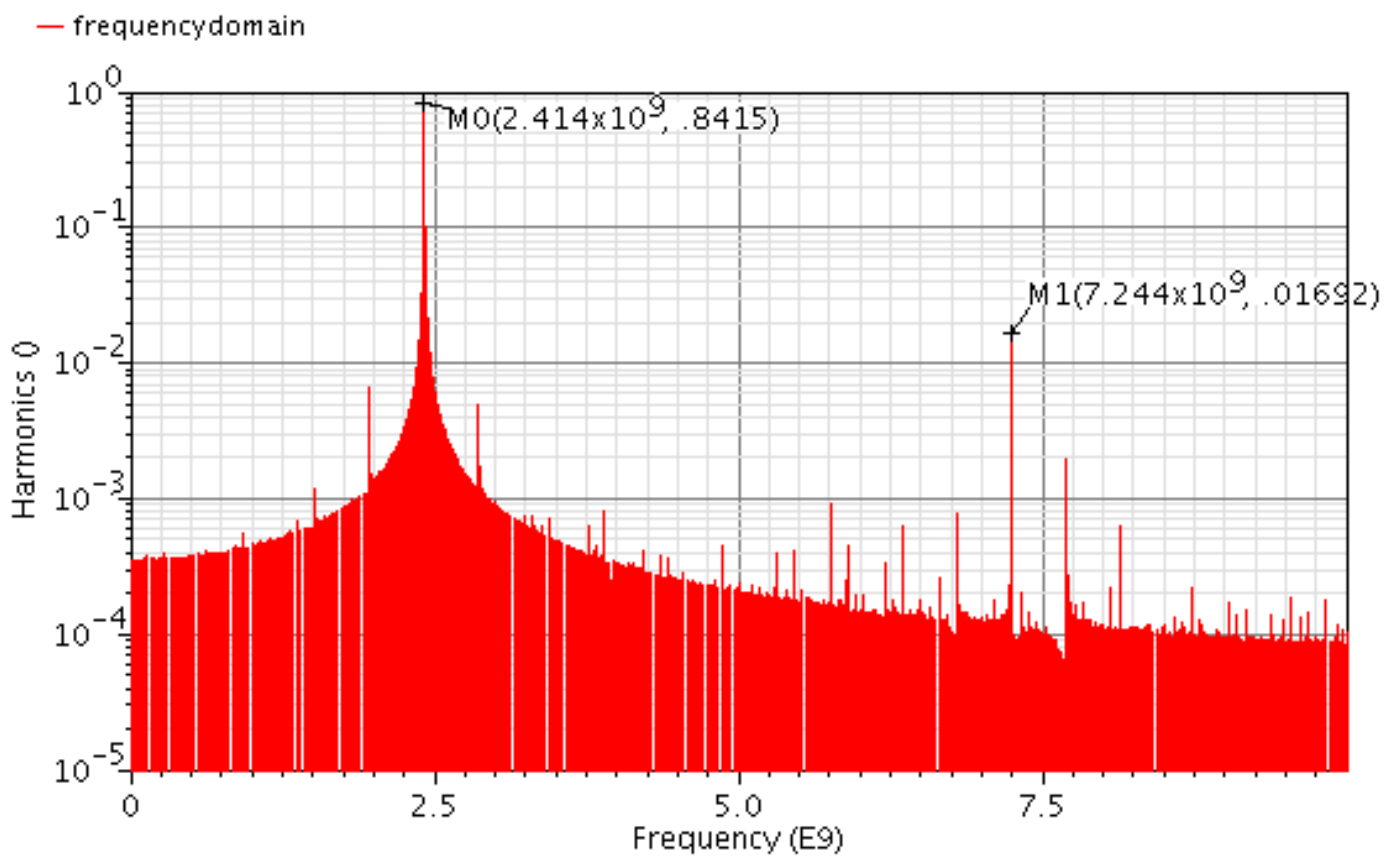

(a) 


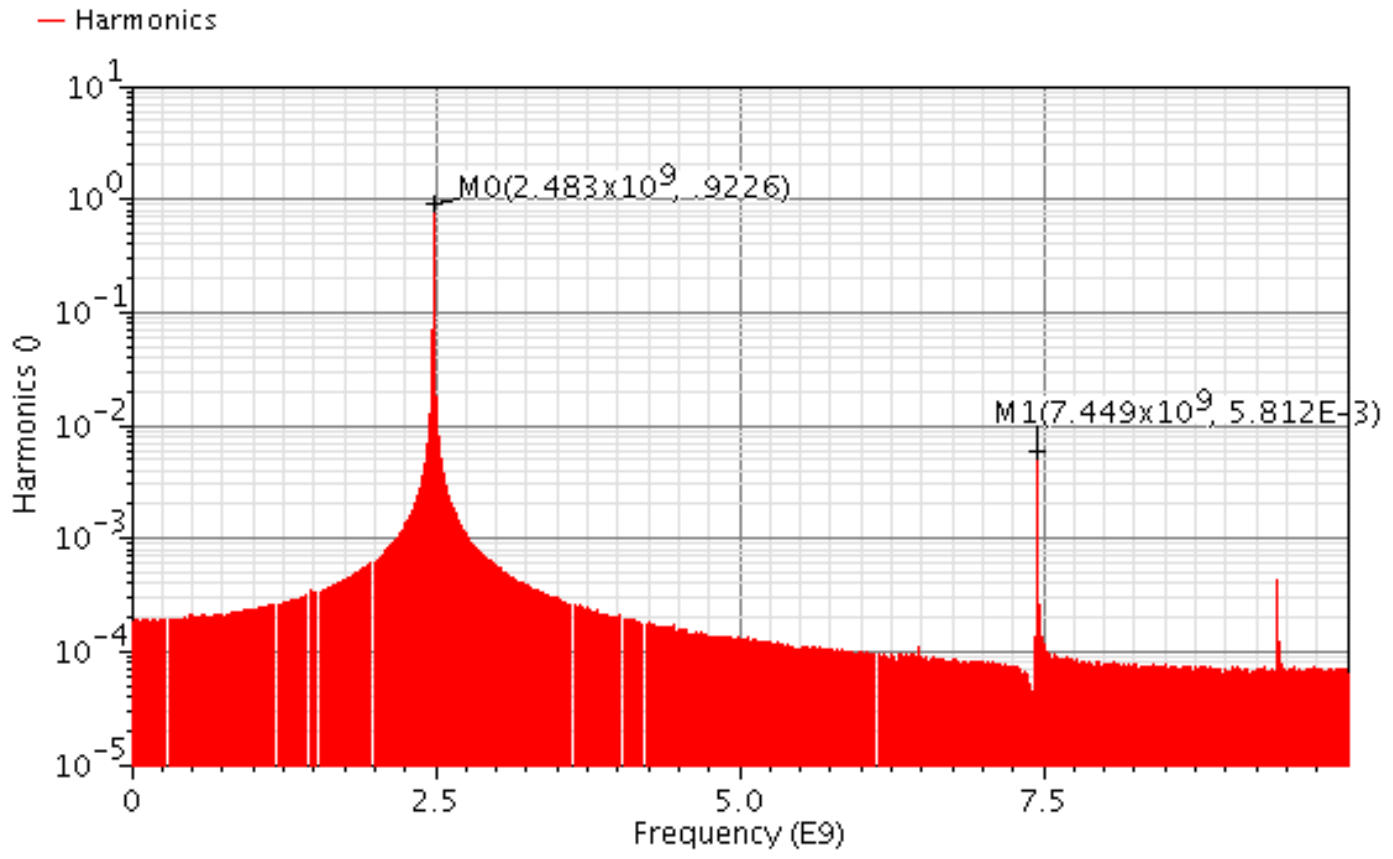

(b)

Expressions

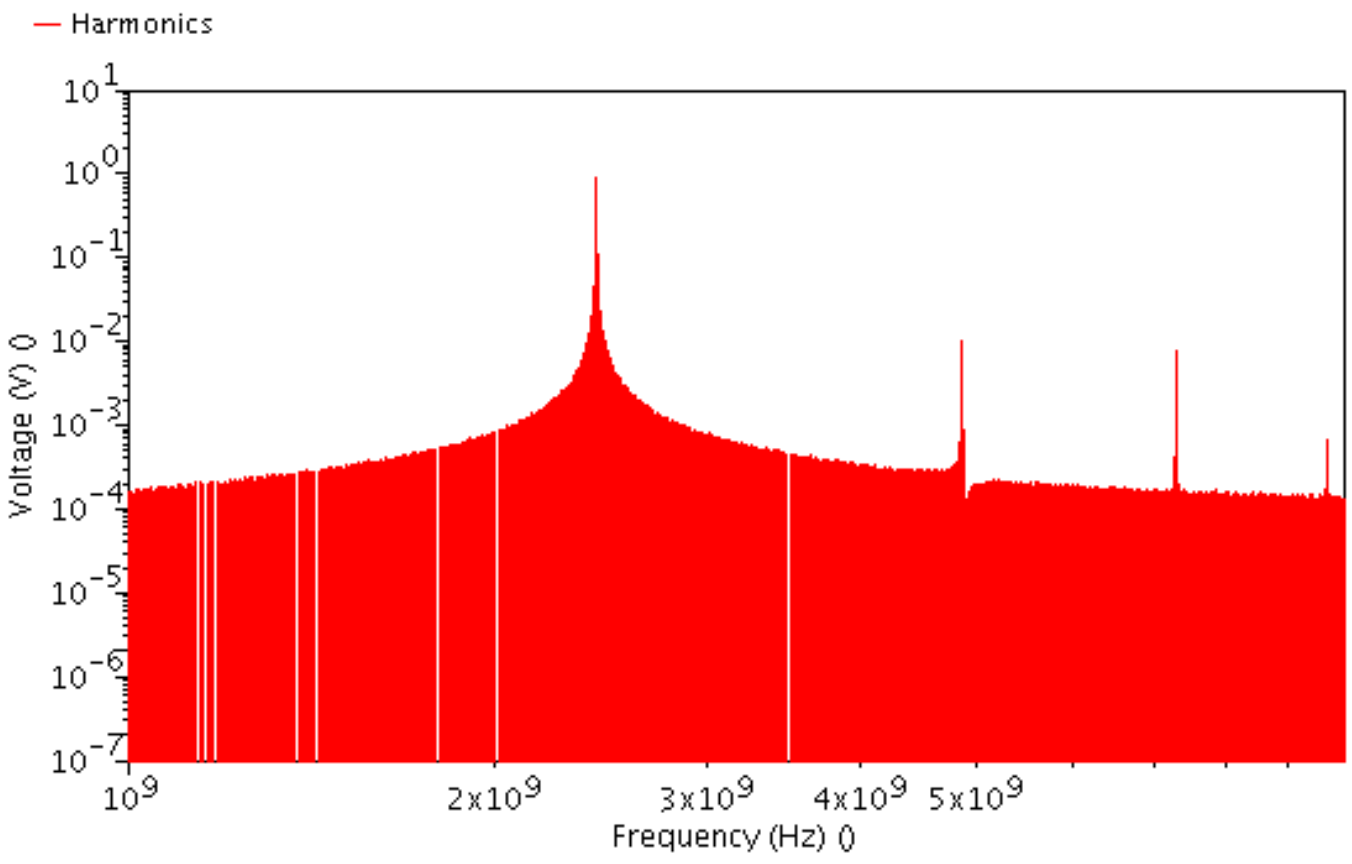

(c)

Figure 3.12: Harmonic simulation of (a) VCO1 (b) VCO2 (c) VCO3 for Experiment II. 
In Experiment II, quite similar results were achieved. Third harmonics are dominant in VCO1 and VCO2, but the second harmonic is introduced in VCO3. In VCO2, THD is reduced to less than one third when compared with VCO1. In VCO3, because the second harmonic is introduced, harmonic components are slightly increased. As concluded in Experiment I, the variation is caused by the $R C$ network. However, when compared with VCO1, it still shrinks the value of THD by half.

From the analysis above, it can be concluded that the transformer does offer advantages in reducing the nonlinearity. The $R C$ feedback network may influence the harmonics, but it is not always beneficial. However, when compared with the transformer, variations in harmonics caused by the $R C$ feedback network are fairly small, which may be ignored.

\subsubsection{Transfer Function}

Because we care less about the closed-loop gains in lower and higher frequencies, only closed-loop gains between $1 \mathrm{GHz}$ to $10 \mathrm{GHz}$ are considered. The simulation results of the transfer function of the three topologies for Experiment I and II are shown in Figure 3.13(a) and Figure 3.13(b), respectively.

To evaluate the narrowness of a transfer function, the factor $K$ has been defined to determine the integral of the closed-loop gain from the fundamental frequency to the fourth harmonic, which is represented by

$$
K=\int_{f_{0}}^{4 f_{0}}|H(f)| d f
$$

where $|H(f)|$ is the magnitude of the closed loop gain and $f_{0}$ is the fundamental frequency or center frequency. From the simulations, the transfer functions are approximated to converge to 0 when $f \rightarrow 0$ and $f \rightarrow \infty$. Note that $K$ does not have any physical meaning; it merely shows how narrow the bandwidth of the transfer function is and how low the peak amplitude of the transfer function is. Results of $K$ are concluded in Table 6 and Table 7 respectively for both experiments. 
Table 6: Simulation results for $\mathrm{K}$ value for Experiment I

\begin{tabular}{c|cc}
\hline & Peak Value & K \\
\hline VCO1 & 13.39 & $2.76 \mathrm{E} 9$ \\
VCO2 & 7.98 & $1.62 \mathrm{E} 9$ \\
VCO3 & 0.2387 & $1.36 \mathrm{E} 7$ \\
\hline
\end{tabular}

Table 7: Simulation results for K value for Experiment II

\begin{tabular}{c|cc}
\hline & Peak Value & K \\
\hline VCO1 & 10.57 & $2.16 \mathrm{E} 9$ \\
VCO2 & 4.534 & $5.92 \mathrm{E} 8$ \\
VCO3 & 0.2366 & $1.36 \mathrm{E} 7$ \\
\hline
\end{tabular}

Expressions

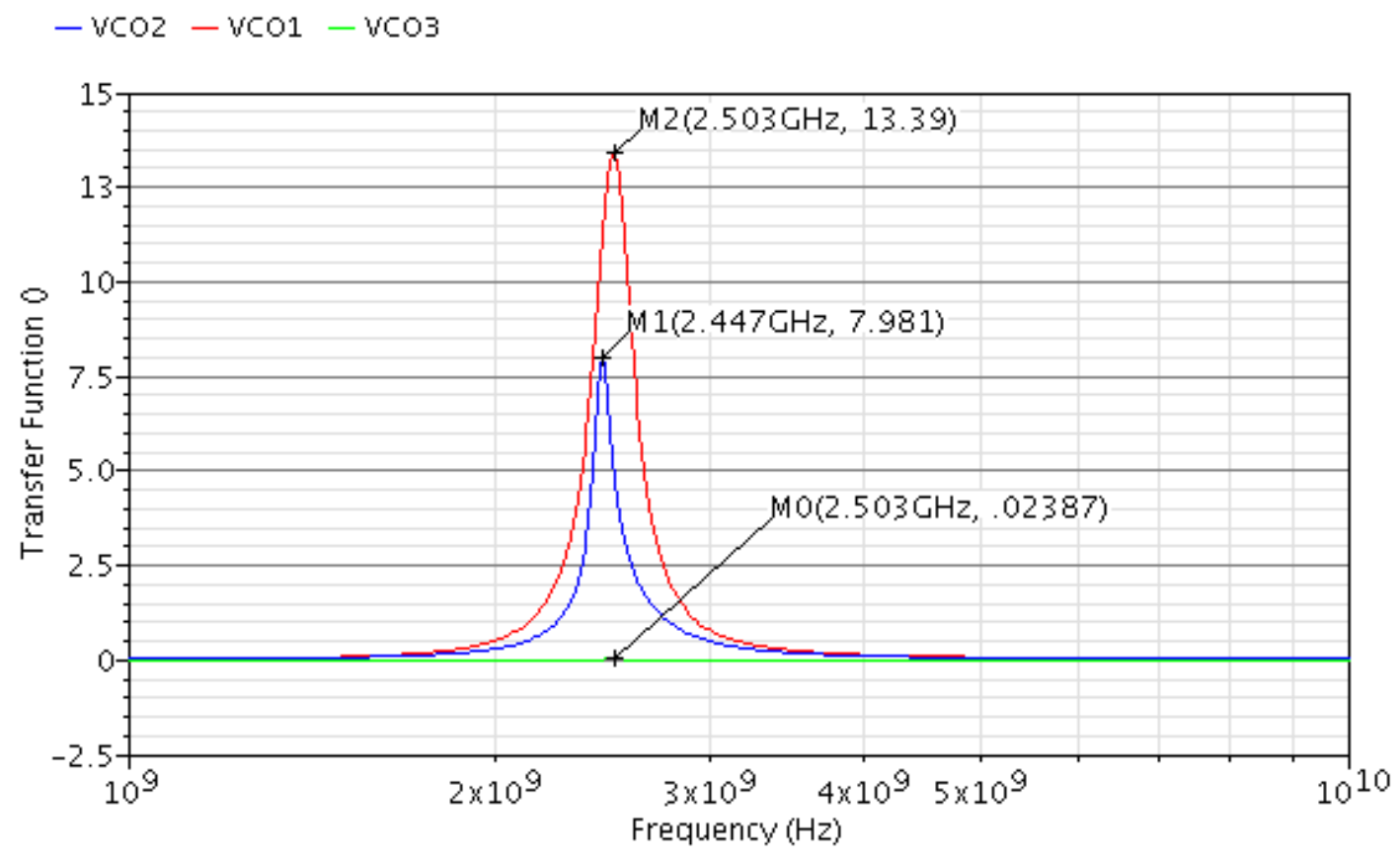

(a) 


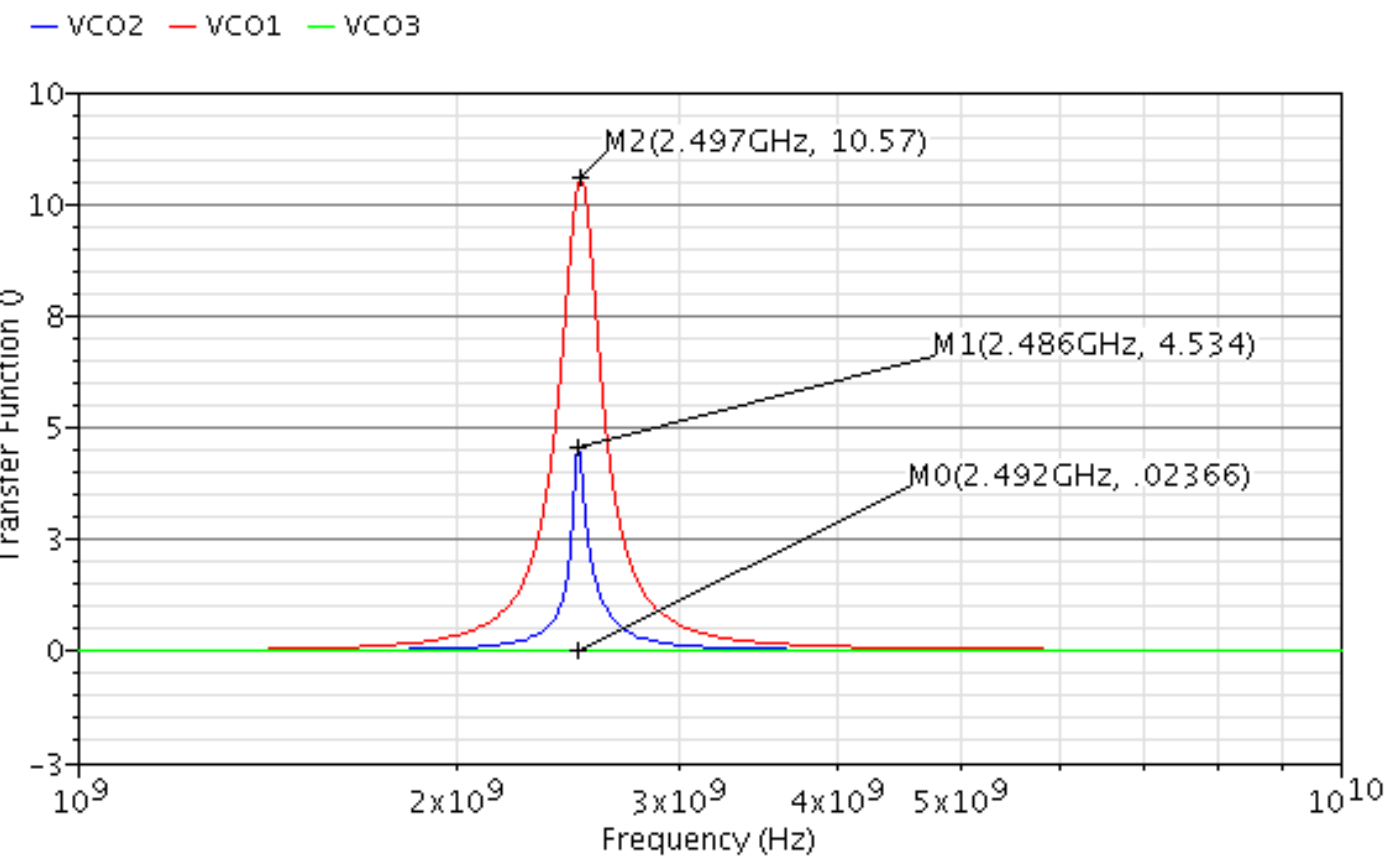

(b)

Figure 3.13: Comparison of transfer functions for (a) Experiment I (b) Experiment II.

Table 8: Simulation results for phase noise performance for Experiment I

\begin{tabular}{l|c}
\hline & Phase Noise $@ \mathbf{1 M H z}(\mathbf{d B c} / \mathrm{Hz})$ \\
\hline VCO1 & -121.9 \\
\hline VCO2 & -124.5 \\
\hline VCO3 & -126.5 \\
\hline
\end{tabular}

Table 9: Simulation results for phase noise performance for Experiment II

\begin{tabular}{c|c}
\hline & Phase Noise $@ \mathbf{1 M H z}(\mathbf{d B c} / \mathbf{H z})$ \\
\hline VCO1 & -122.2 \\
\hline VCO2 & -125.6 \\
\hline VCO3 & -126.5 \\
\hline
\end{tabular}




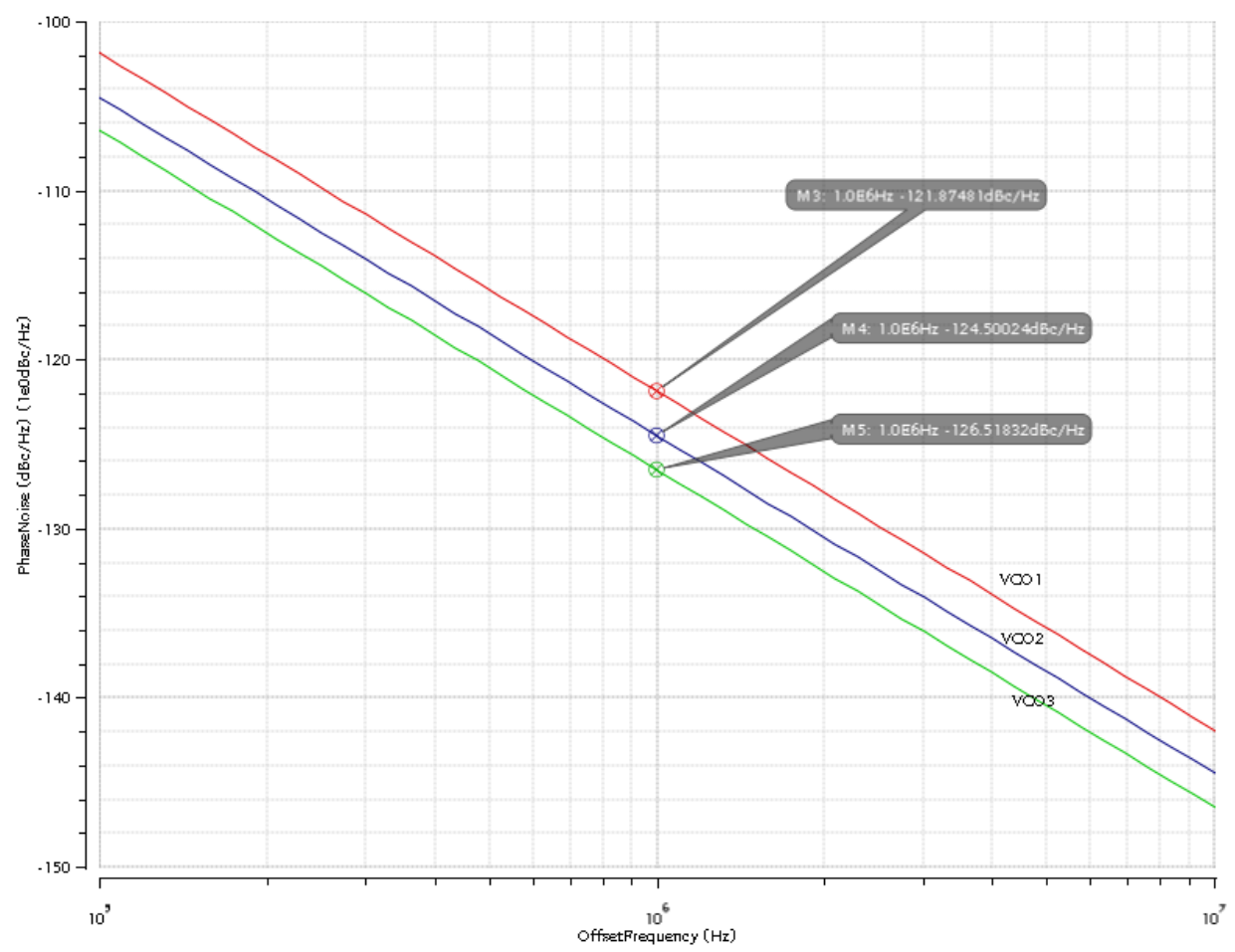

(a)

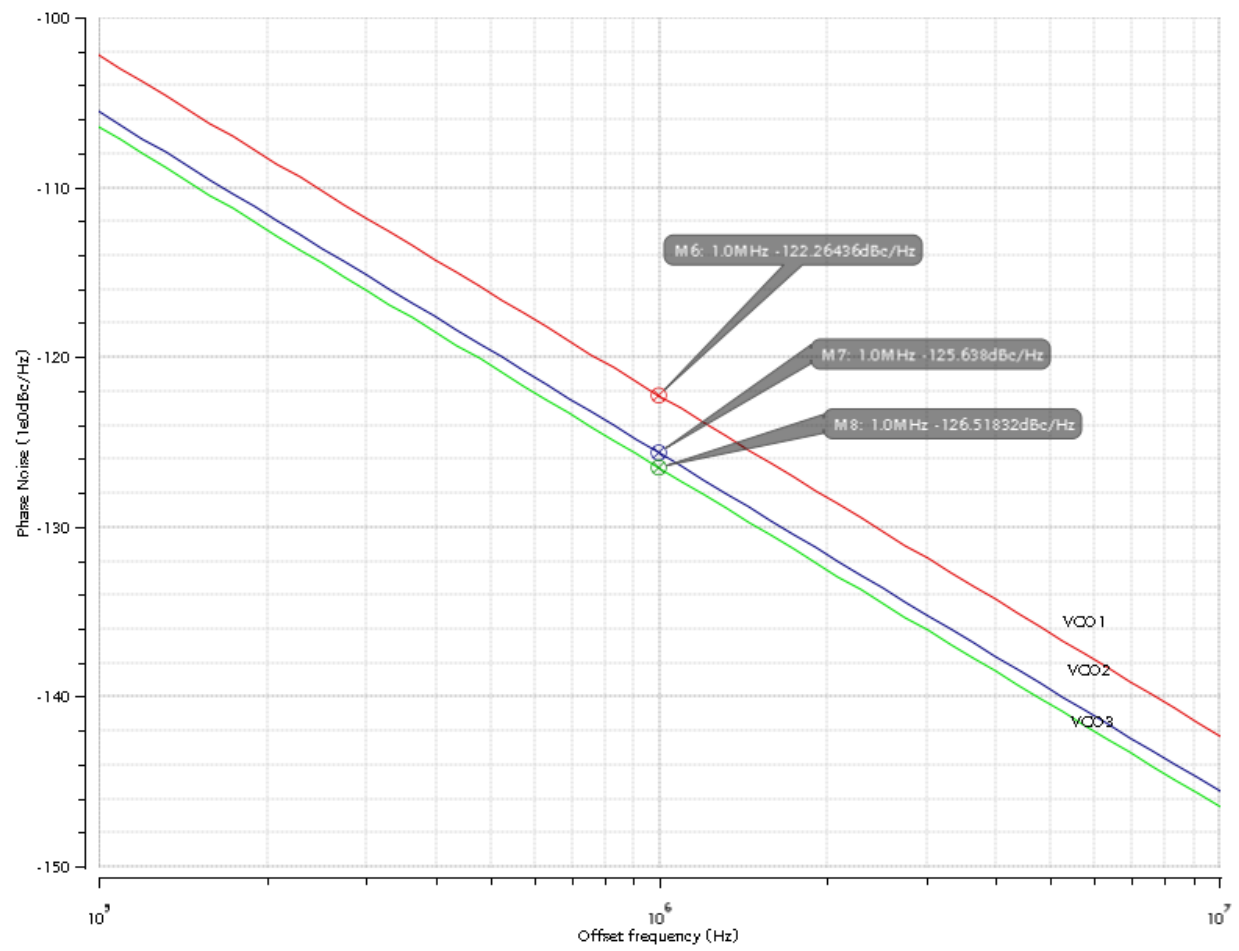

(b)

Figure 3.14: Phase noise comparison of $\mathrm{VCO} 1, \mathrm{VCO} 2$ and $\mathrm{VCO} 3$ 
In comparing $\mathrm{VCO} 2$ and $\mathrm{VCO} 3$, it is apparent that with the contribution of the $R C$ feedback network, the magnitude and the bandwidth of the transfer function is severely reduced. When comparing VCO2 with VCO1, it can be seen that the transformer may also benefit the transfer function; however, its influence is less than that of the $R C$ feedback network.

\subsubsection{Phase Noise}

From the analysis above, it can be seen that the proposed circuit has the ability to reduce both transfer function and harmonics efficiently. Therefore, it is not difficult to predict that the phase noise performance should be improved.

Simulation results for the phase noise performance of Experiment I and II are shown in Figure 3.14. Phase noise performances at $1 \mathrm{MHz}$ offset are illustrated in Table 8 and Table 9.

In both experiments, VCO3 offers the best phase noise performance among the three circuits. This is not surprising to see in Experiment I, as VCO3 had the best performance in both transfer function and harmonics. However, in Experiment II, although the harmonics in VCO3 are higher than they are in VCO2, the large decrement in transfer function is still dominant, resulting in VCO3's best phase noise performance.

\subsubsection{Analysis on Flicker Noise}

After exploring how the proposed circuit benefits harmonics and transfer function under the condition that flicker noise is turned off in the simulator, flicker noise coefficient is turned on again in the simulator to see how flicker noise affects the phase noise performance in this circuit.

Figure 3.15 shows the phase noise performance when Experiment I is repeated when flicker noise is turned on. Other parameters are kept same. Table 10 concludes the phase noise performance.

The differences between different flicker noise conditions in VCO1, VCO2 and VCO3 are $7.4 \mathrm{~dB}, 3.1 \mathrm{~dB}$ and $0.7 \mathrm{~dB}$ respectively. Such differences are caused by the flicker noise. VCO1 shows worst resistance to flicker noise, while VCO3 shows best 


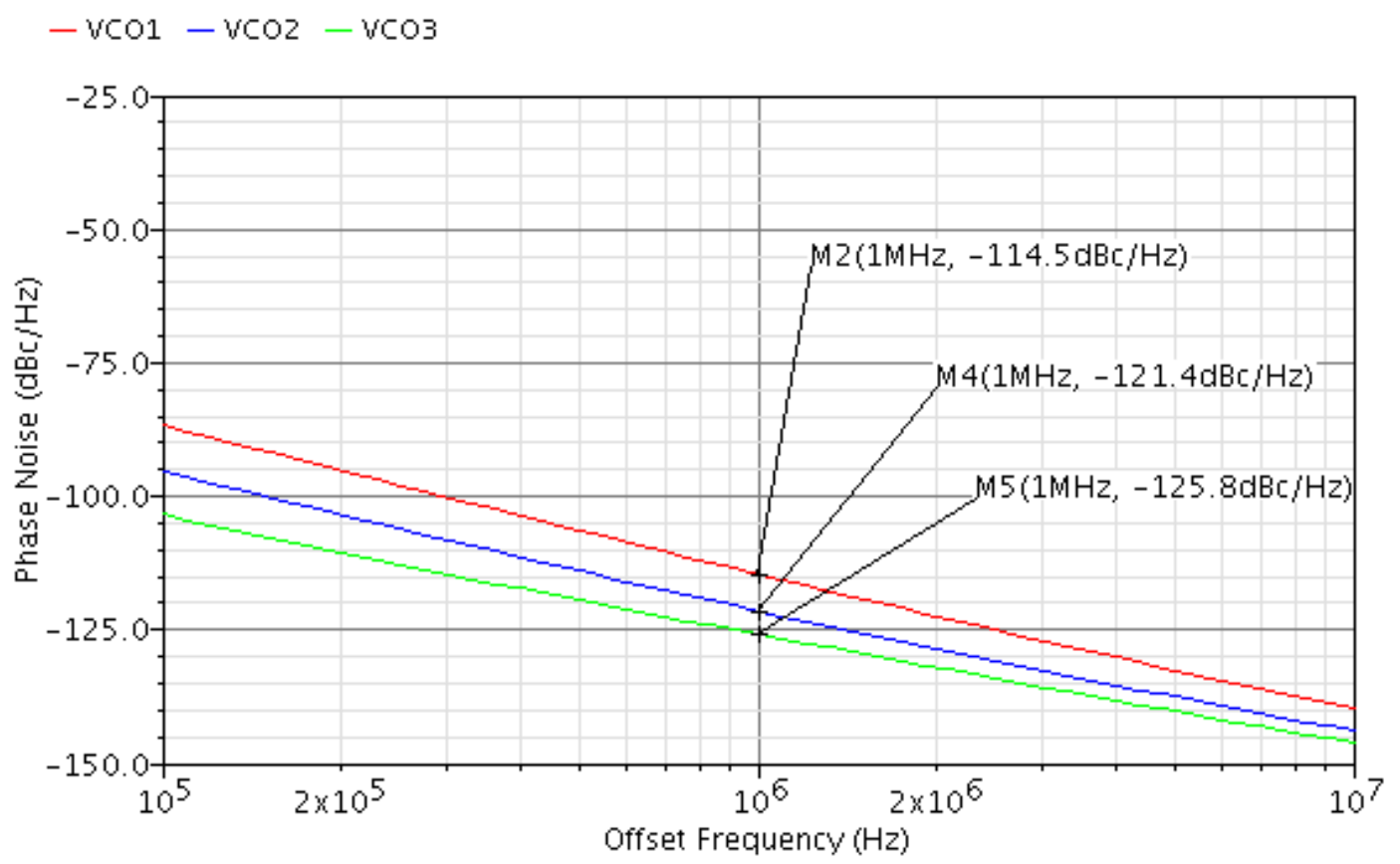

Figure 3.15: Phase noise comparison of VCO1, VCO2 and VCO3 when flicker noise is introduced.

Table 10: Simulation results for phase noise performance for Experiment I under different flicker noise condition

\begin{tabular}{c|c|c}
\hline \multirow{2}{*}{} & \multicolumn{2}{|c}{ Phase Noise @ $\mathbf{1 M H z}(\mathbf{d B c} / \mathbf{H z})$} \\
\cline { 2 - 3 } & Flicker noise off & Flicker noise on \\
\hline VCO1 & -121.9 & -114.5 \\
\hline VCO2 & -124.5 & -121.4 \\
\hline VCO3 & -126.5 & -125.8 \\
\hline
\end{tabular}

performance in resisting transferring flicker noise into phase noise. This is because the coupled inductors and $\mathrm{RC}$ filter existing in the circuit can efficiently help filtering out part of the flicker noise. This benefit further proves the advantage of the proposed circuit in phase noise performance.

\subsection{Conclusion}


The above chapter has explored the mechanism on phase noise generation. Based on the research, it is found that the phase noise performance is determined by the transfer function and harmonic components of a VCO circuit.

According to the studies, VCO design guidelines have been concluded in guiding low phase noise VCO design. Based on which, a novel VCO topology has been proposed to reduce harmonic components and decrease the magnitude and the bandwidth of the transfer function, aiming to enhance the phase noise performance. According to the simulation results, this topology managed to reduce harmonic components and decrease the magnitude and the bandwidth of the transfer function. At the same time, the circuit also shows advantage in suppressing the up-conversion of flicker noise. Under the same conditions (e.g., tank, active devices), phase noise was improved as compared with the reference designs. 


\section{Chapter: Circuit Design}

\subsection{Introduction}

This chapter demonstrates a VCO circuit design based on the proposed topology. The VCO is expected to have a center frequency of $2.4 \mathrm{GHz}$ and a tuning range of $10 \%$. Tank design, including the inductor and varactor design, is first introduced, followed by the design on the transformer and $R C$ network designs. In each part, consideration of layout is taken into account. Lastly, simulation results are present.

\subsection{Tank}

In LC tank-based VCOs, the phase-noise performance is highly influenced by the tank. The higher the $Q$ is, the better the phase-noise performance is [27]. This can be proved by Leeson's empirical (10) and a huge number of references.

In this work, single-ended $L$ is chosen as $2 \mathrm{nH}$ and a single-ended $C$ is chosen from 1.9 to $2.4 \mathrm{pF}$. This is determined alongside several considerations. Although, theoretically, a much larger $L$ would provide better phase-noise performance and save more power, in reality, a layout with a larger $L$ usually brings more parasitic resistance, thus lowering the quality factor of the tank. Also, it may cause problems in designing the transformer as a result of the chip area consideration.

\subsubsection{Inductor Design}

A quality factor, by definition, is a dimensionless parameter that describes how underdamped an oscillator is. Further details about quality factors will be discussed in Appendix B. Higher factors indicate lower rates of energy loss relative to the stored energy of the resonator. In an LC tank, the quality factor is mainly determined by the quality factor of the inductor. For on-chip inductors, energy loss is caused by parasitic resistance. Previously, quality factors of on-chip inductors usually ranged from 3 to 5; however, now, with the progression of technology, the quality factors of on-chip inductors can reach 30 or even higher [38-41]. Different layout methods may also contribute to higher quality factors [42]. 


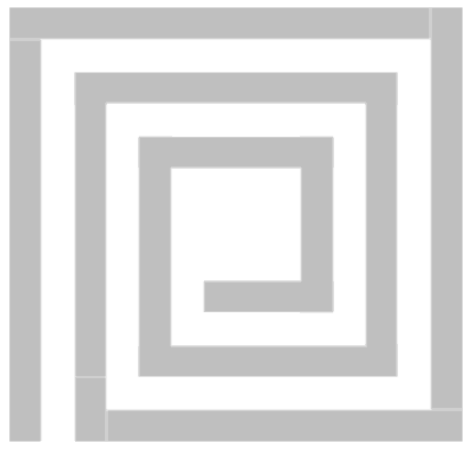

(a)

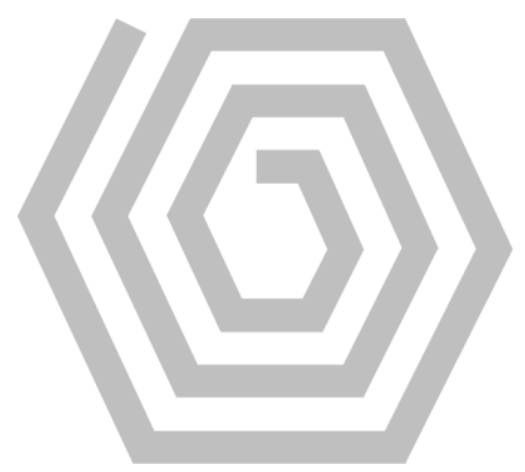

(b)

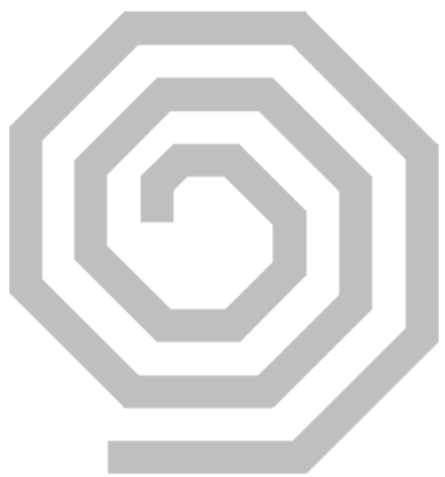

(c)

Figure 4.1: Single-end planar spiral inductor layout methods. (a) Square (b) Hexagonal (c) Octagonal

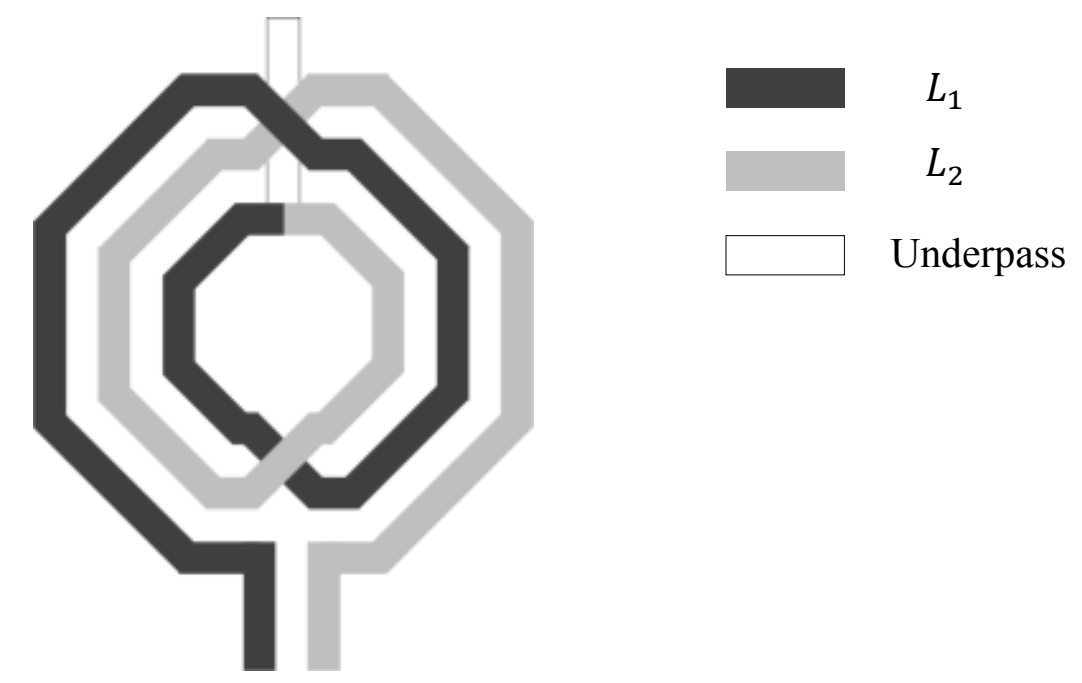

Figure 4.2: Differential symmetric layout method.

Figure 4.1 shows three different layout methods for single-end planar spiral inductors. The square version is popular because it is easy to generate in layout tools. Polygonal spirals with more than four sides are one example; hexagonal and octagonal inductors are also widely used.

However, in a differential circuit, differentially driven symmetric inductors (Figure 4.2) are more frequently used. This method not only realizes a nearly perfectly symmetrical layout, but it also improves the quality factor by differential excitation. In practice, the improvement would be lower, but still would provide 50\% greater Q [42]. 


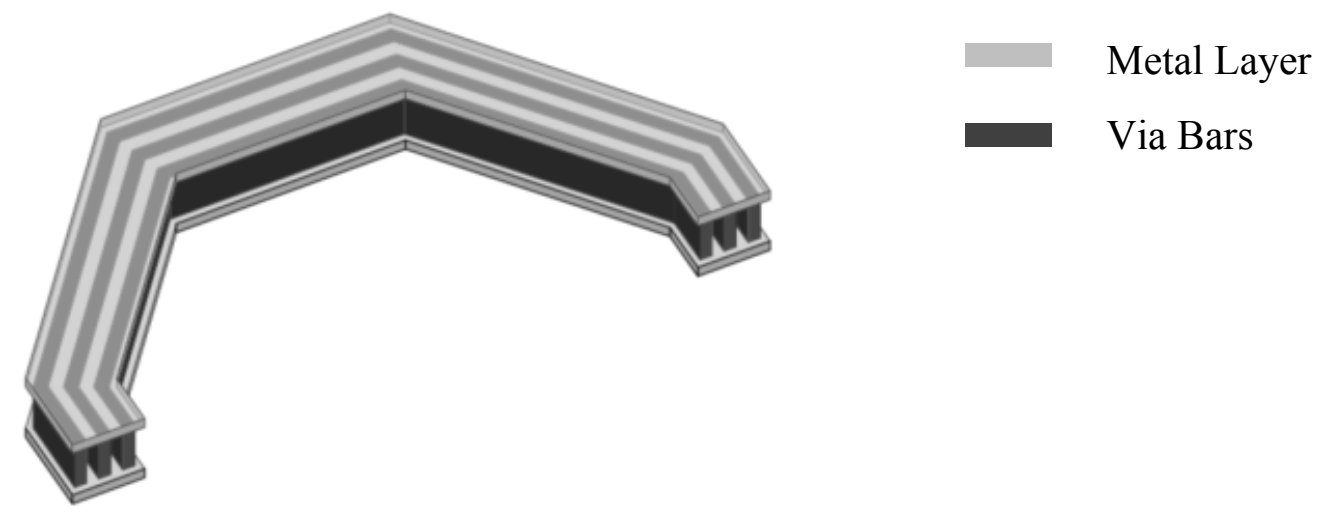

Figure 4.3: Multilayer layout method [5].

With the process in technology, multilayer inductors became more dominant because they offer higher $Q$ s. That is, although the thickness of the inductors has less of an influence on the inductance, they severely decide the resistance of the inductors. The thicker the layer, the lower the resistance, gave a higher $L_{s} / R_{s}$ ratio, and thus better performance. In practice, maximizing thickness is realized by connecting two layers with VIA bars (see Figure 4.3).

In this work, a differential multilayer structure has been employed. Because the thickness of the layer is certain, a large width and small turn-spacing minimize the parasitic resistance. After optimization, the quality factor at a desired frequency range $(2.2-2.5 \mathrm{GHz})$ achieves around 21. Inductance is approximately $2 \mathrm{nH}$, while the resistance ranges from 1.3-1.5 Ohm. Simulation results are shown in Figure 4.4. Details regarding the device's sizes are shown in Appendix E

\subsubsection{Varactor Design}

There are several ways to form a varactor, diode varactor, pMOS varactor, and MOS varactor [5] [43]. MOS varactors, which allow for a better overall VCO performance, are more often favored.

MOS is a transistor with four terminals. However, when the source and drain are shorted and biased, the transistor could be used as a varactor because the capacitance of 


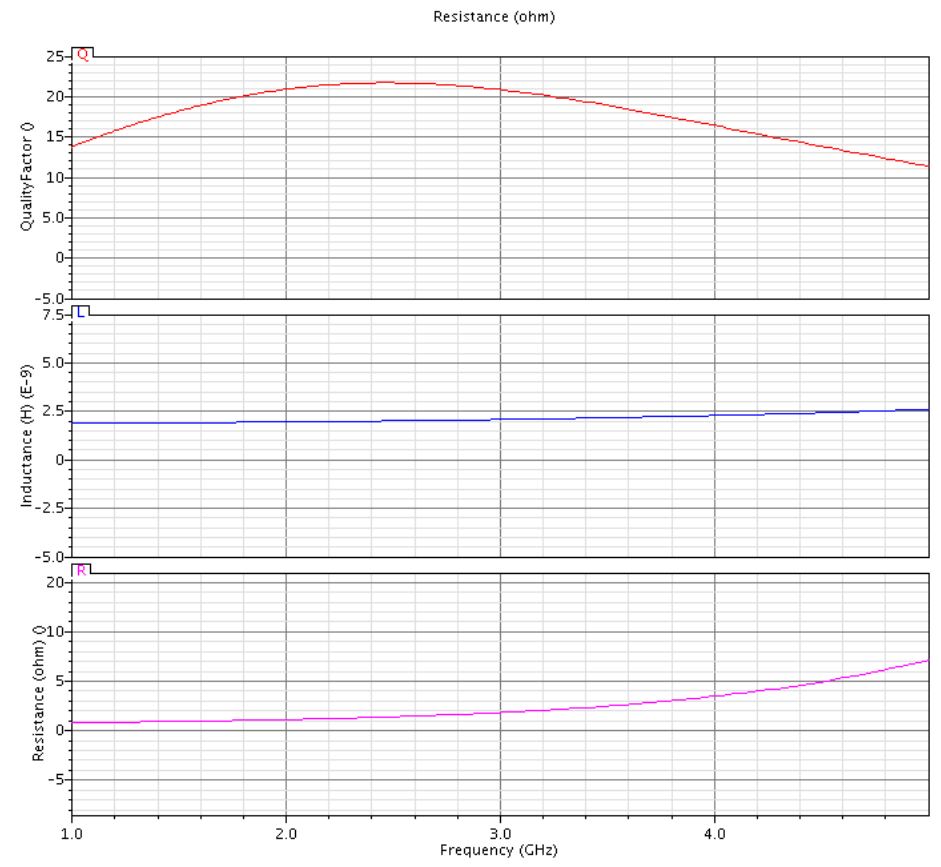

Figure 4.4: Simulation results for single-end inductors.

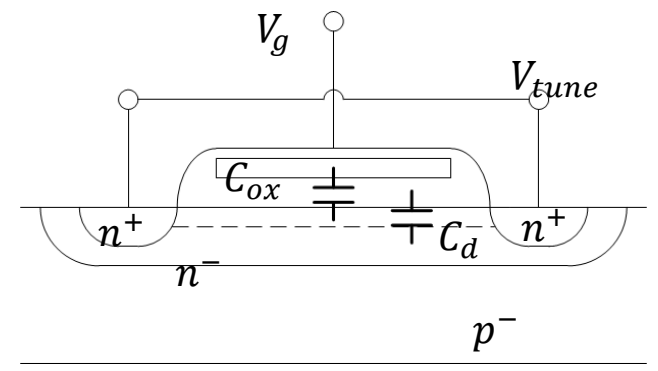

Figure 4.5: Side view of MOS varactor

the bottom plate changes with bias. Figure 4.5 shows a MOS varactor that is created by placing the $n^{+}$diffusion regions of an NMOS device in an n-well region.

Define

$$
V_{g d}=V_{g}-V_{\text {tune }}
$$




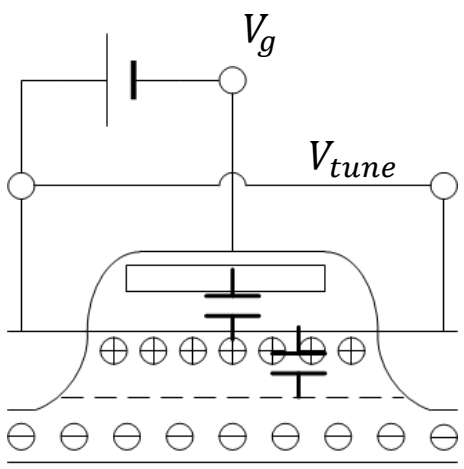

(a)

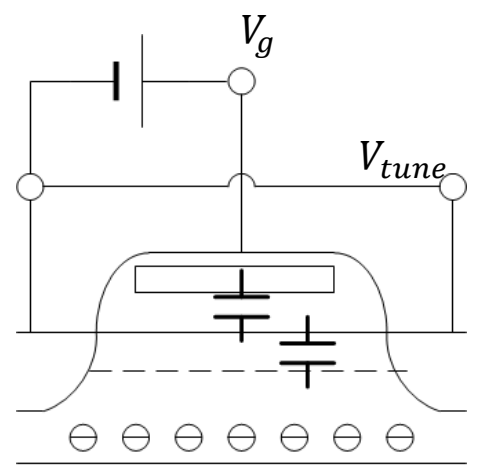

(b)

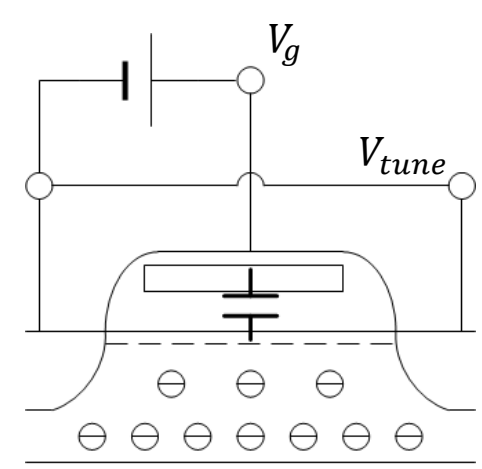

(c)

Figure 4.6: Varactors working in (a) Inversion region (b) Depletion region (c) Accumulation region.

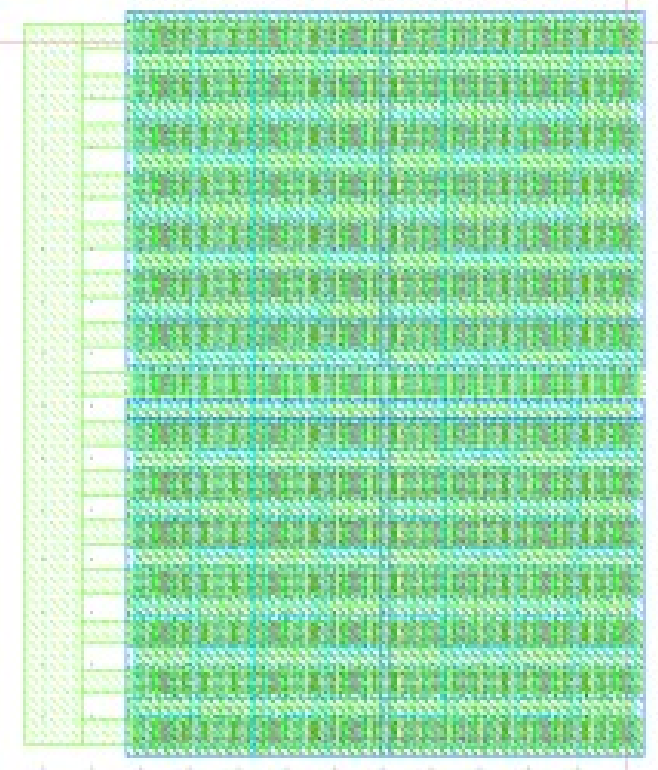

Figure 4.7: Layout for varactor arrays.

When $V_{g d}$ is changed from negative to positive, the device operates from the inversion region to the depletion region, and, finally, to the accumulation region (see Figure 4.6). 


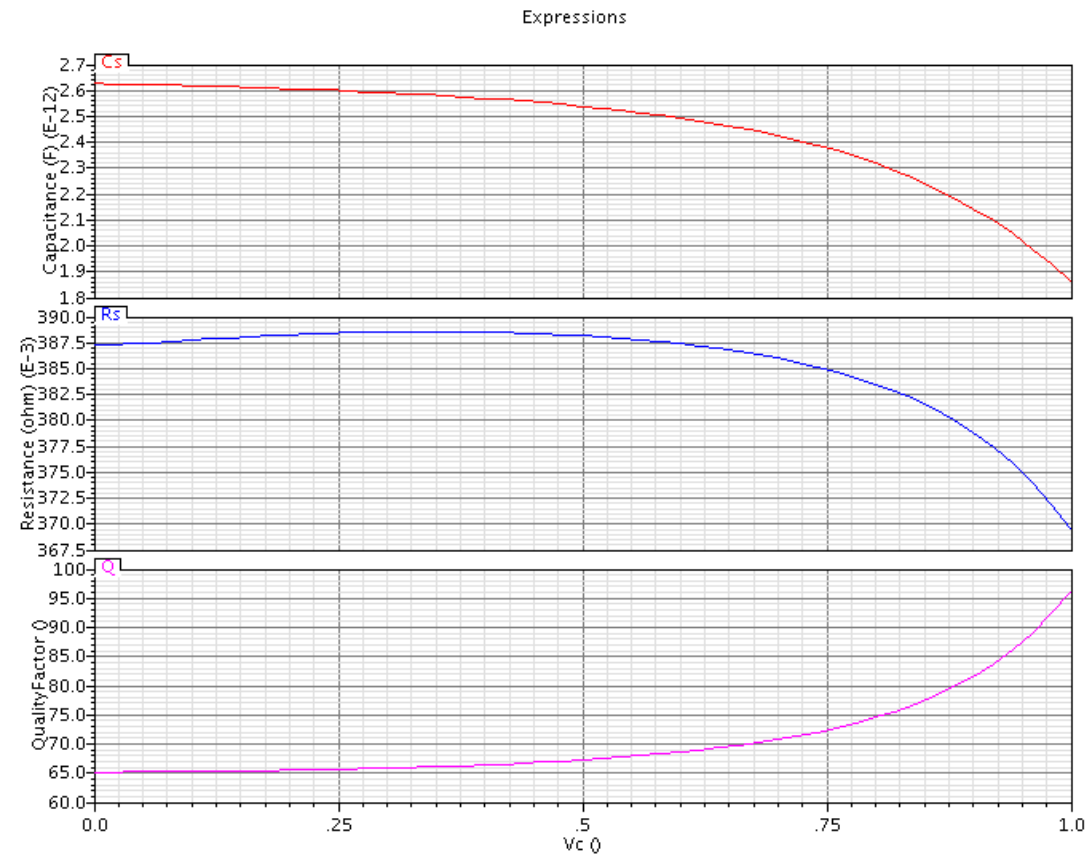

Figure 4.8: Simualtion results for the characterization of varactor arrays.

When the varactor works only in the depletion and accumulation regions, it is called an A-MOS varactor. Usually linearity is better in this region than in the inversion region. In this design, differential A-MOS varactors are used. To maximize the quality factor and $\mathrm{Cmax} / \mathrm{Cin}$, and to minimize the parasitic resistance, arrays of smallest-unit AMOS fingers are placed in parallel to each other as shown in Figure 4.7. Parasitic resistance is around $0.37 \mathrm{Ohm}$ within the frequency range. Capacitance varies from 1.92.4pF. Simulations are shown in Figure 4.8.

\subsection{Transformer}

There are several ways to design on-chip transformers [44]. Figure 4.9 shows three different realizations of on-chip transformers: tapped, interleaved, and stacked. Tapped transformers have the advantage of giving high self-inductance and low port-to-port capacitance. However, the trade-off is that it produces a relatively low mutual coupling because of spatial separation. Usually the coupling coefficient is $k \in(0.3,0.5)$. 


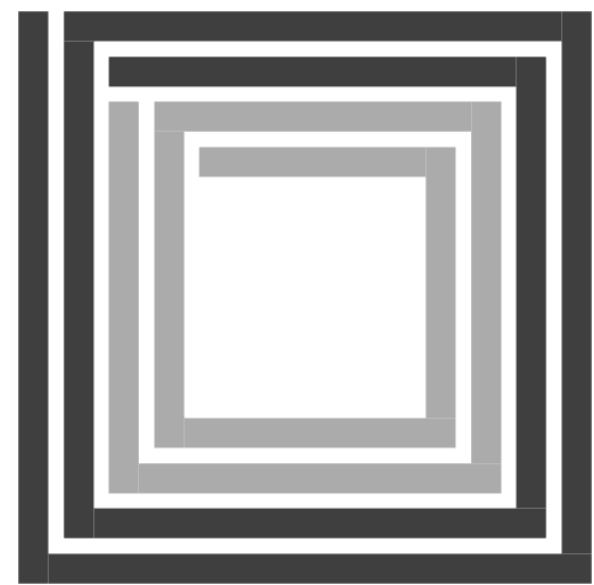

(a)

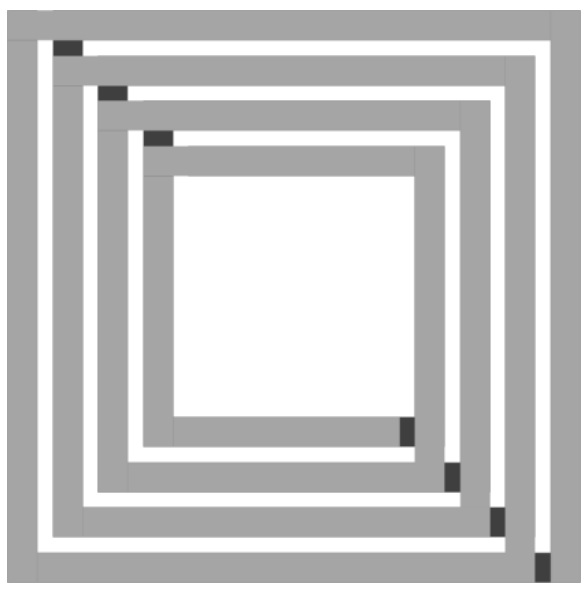

(c)

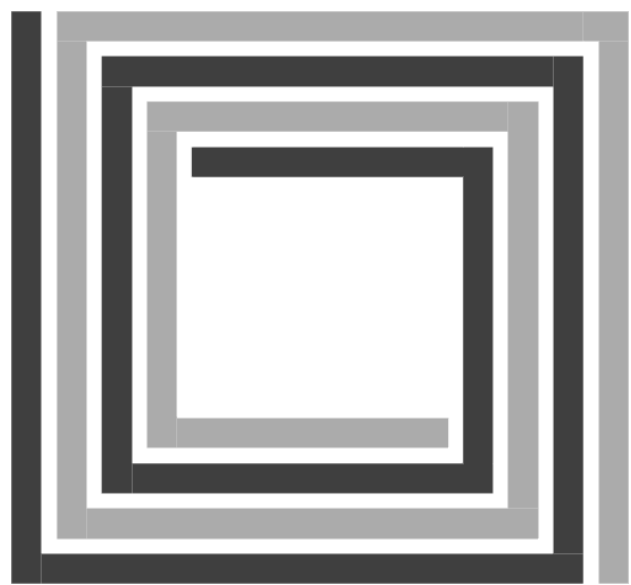

(b)

(d)

Figure 4.9: Layout method for on-chip transformers. (a) Tapped (b) interleaved (c) Stacked

Table 11: Comparison between different layout methods for on-chip transformers

\begin{tabular}{c|ccc}
\hline & Self-inductance & Port-to-port capacitance & coupling coefficient \\
\hline Tapped & High & Low & $(0.3,0.5)$ \\
Interleaved & Low & Low & $(0.7,0.8)$ \\
Stacked & Very high & High & 0.9 \\
\hline
\end{tabular}

Interleaved transformers offer a relatively high coupling coefficient $(k \approx 0.7)$ and low port-to-port capacitance. However, the self-inductance is lower.

Stacked transformers are realized in multilayers, giving high self-inductance and a very high coupling coefficient ( $k$ may reach 0.9 ). The trade-off is the high port-to-port capacitance, which is equivalent to a low self-resonance. This can be adjusted by 


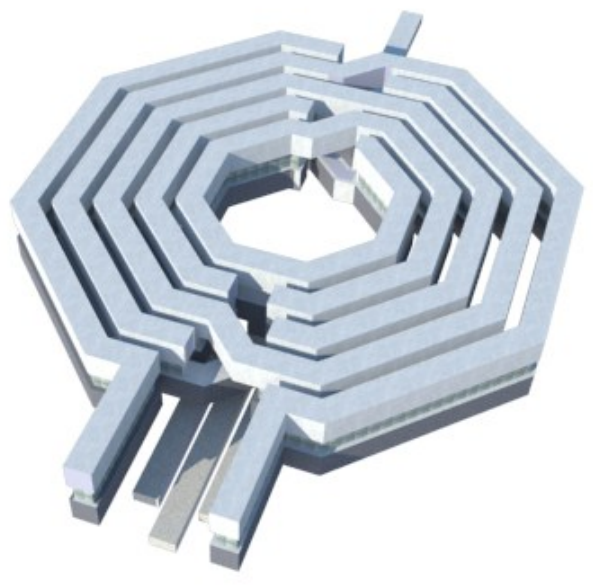

Figure 4.10: 3D view on proposed transformer

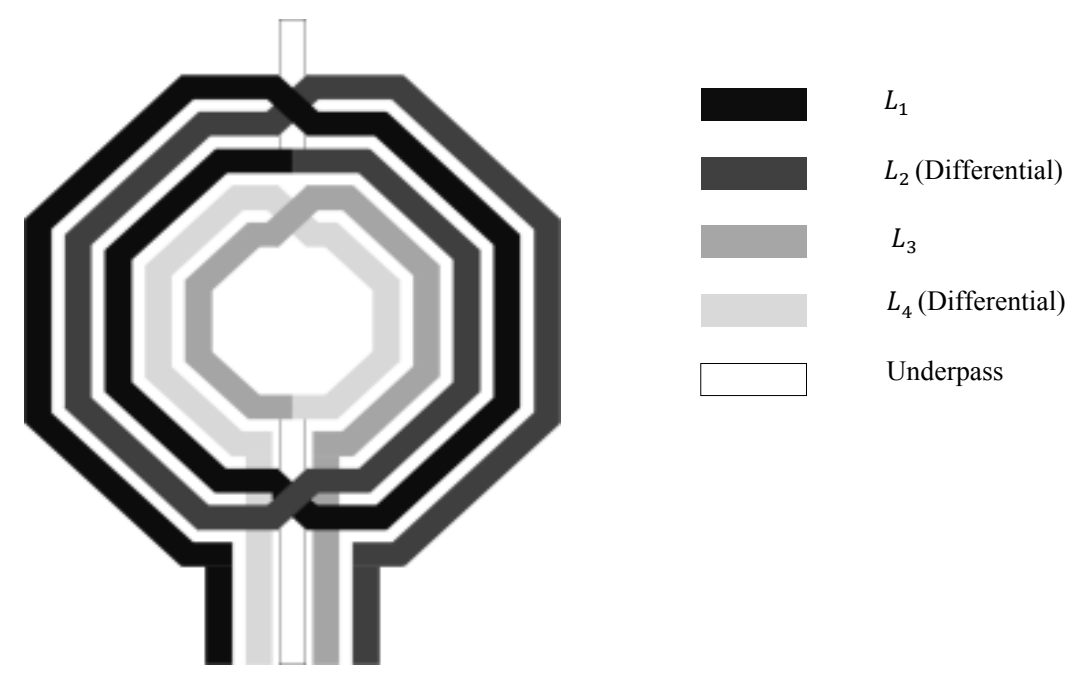

Figure 4.12: Layout method for the proposed transformer

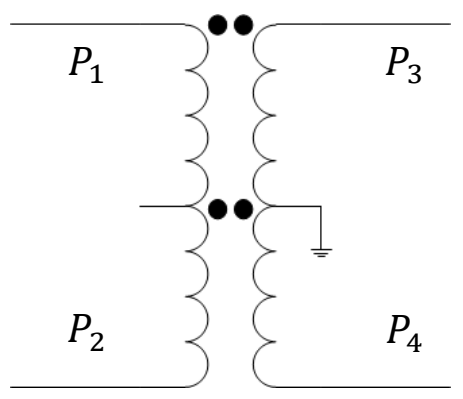

Figure 4.13: 4-port transformer 
Table 12: Parameters for transformer1 and transformer2 (Square Spiral)

\begin{tabular}{c|c|c}
\hline \multirow{2}{*}{ Parameter } & Value \\
\hline \multirow{3}{*}{$\begin{array}{c}\text { Primary } \\
\text { Inductor }\end{array}$} & Outer dimension & $300 \mathrm{um}$ \\
\cline { 2 - 3 } & Width & $8.5 \mathrm{um}$ \\
\cline { 2 - 3 } & Space & $5 \mathrm{um}$ \\
\hline \multirow{3}{*}{ Secondary } & Turn & 3 \\
\cline { 2 - 3 } Inductor & Outer dimension & $219 \mathrm{um}$ \\
\cline { 2 - 3 } & Width & $8.5 \mathrm{um}$ \\
\cline { 2 - 3 } & Space & $5 \mathrm{um}$ \\
\hline
\end{tabular}

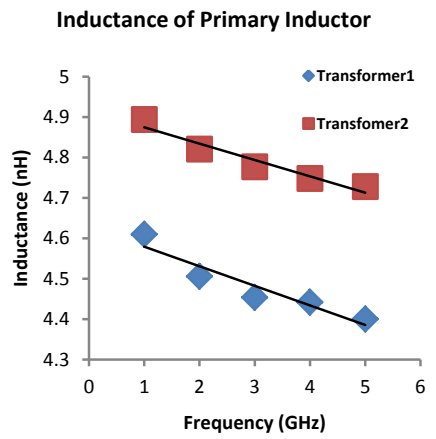

(a)

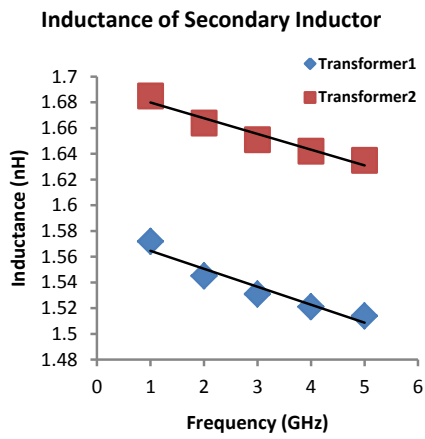

(b)

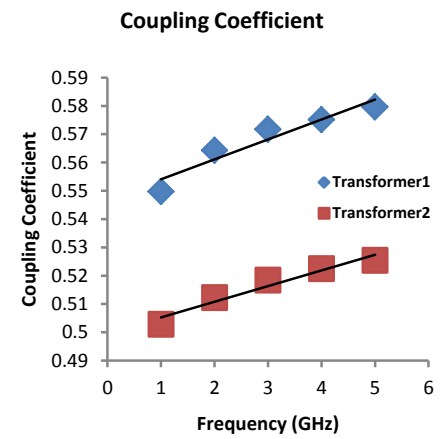

(c)

Figure 4.14: Simulation results for Transformer1 and Transformer2 using ASITIC

(a) Primary inductor (b) Secondary inductor (c) Coupling coefficient

increasing the distance between the two layers or by changing the center of the two spirals.

In the proposed circuit, high self-inductance is required. High self-inductance, especially for the tank inductor, is to achieve a higher $Q$. The coupling coefficient is not strictly required.

Hence, the tapped type is chosen for the proposed circuit. Since tank inductors and source inductors are both differential, the four inductors can be realized by one transformer with four ports (see Figure 4.13). A top view and 3D view of the final transformer are shown in Figure 4.12 and Figure 4.10 respectively. 


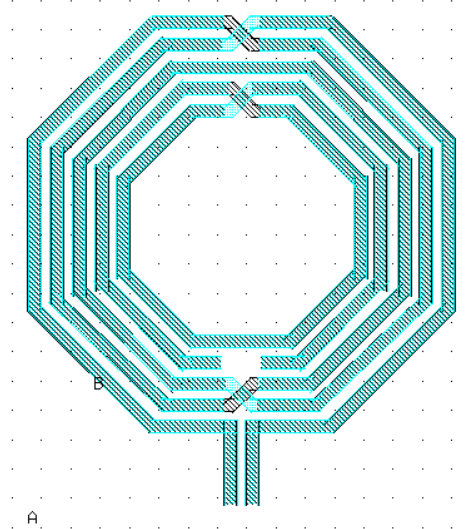

Figure 4.15: Schematic for proposed transformer using ASITIC

Table 13: Parameters for the design symmetrical transformer

\begin{tabular}{c|c|c}
\hline \multirow{2}{*}{ Parameter } & Value \\
\hline \multirow{2}{*}{$\begin{array}{c}\text { Primary } \\
\text { Inductor } \\
\text { (Differential) }\end{array}$} & Outer dimension & $300 \mathrm{um}$ \\
\cline { 2 - 3 } & Width & $8.5 \mathrm{um}$ \\
\cline { 2 - 3 } & Space & $5 \mathrm{um}$ \\
\hline \multirow{2}{*}{$\begin{array}{c}\text { Secondary } \\
\text { Inductor } \\
\text { (Differential) }\end{array}$} & Turn & 3 \\
\cline { 2 - 3 } & Wuter dimension & $219 \mathrm{um}$ \\
\cline { 2 - 3 } & Width & $8.5 \mathrm{um}$ \\
\hline
\end{tabular}

To provide a good short to the ground for the electric field, a ground shielding is placed, using the lower metal [45]. The layout view of the complete transformer with shield is shown in Figure 4.11.

To verify the transformer design, a model has been built up using ASITIC. In this software, "joinshunt" command is used to join multiple spirals in shunt. To verify the accuracy of using "joinshunt" command in simulating the multiply layer transformer, a simple experiment has been carried out to compare the coupling coefficient of a square spiral transformer using the double layer inductor created by "JoinShunt" command (Tranformer2) with the double layer inductor model exiting in the simulator 
Table 14: Parameters for differential dual layer inductors

\begin{tabular}{c|c|c}
\hline \multicolumn{2}{c|}{ Parameter } & Value \\
\hline \multirow{3}{*}{$\begin{array}{c}\text { Differential } \\
\text { Inductor }\end{array}$} & Outer dimension & $300 \mathrm{um}$ \\
\cline { 2 - 3 } & Width & $8.5 \mathrm{um}$ \\
\cline { 2 - 3 } & Turn & 1 \\
\hline
\end{tabular}

Inductance for differential inductors

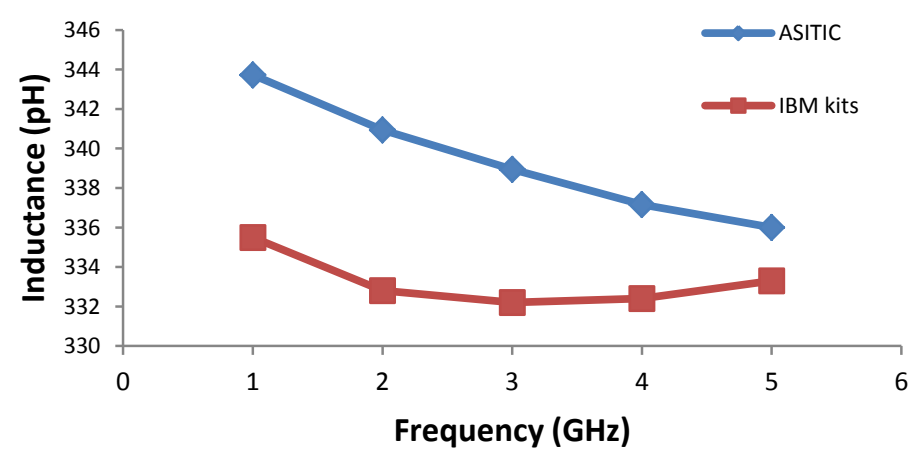

Figure 4.16: Simulation results for the differential inductance of dual layer inductors.

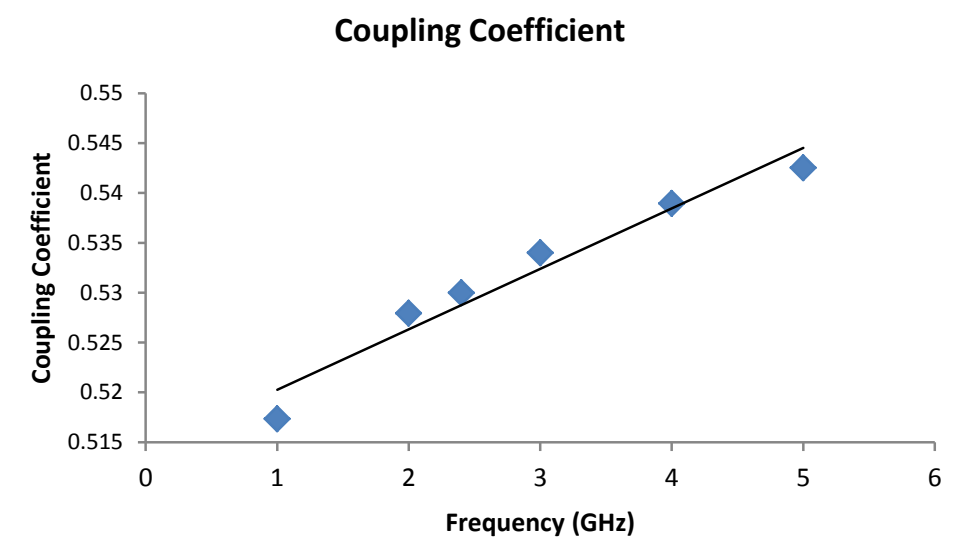

Figure 4.17: Simulation results for the coupling coefficient of proposed transformer

using "joinshunt" command (Transformer1) to the multiply layer transformer model that exists in the software (Transformer2) when other parameters are kept same. Parameters are concluded in Table 12. Results of the inductance and the coupling coefficient are shown in Figure 4.14. 
From Figure 4.14, it can be observed that even though there is variance between the two methods, especially in simulating inductance. However using "joinshunt" command still can roughly predict the coupling coefficient of the transformer using double layer inductors.

To further prove the reliability of the simulator, another experiment has been carried out to compare the inductance of differential dual layer octagonal symmetrical inductor using ASITIC to the model that is provided by the IBM kits because mutual inductance is included in the differential inductors. Parameters are concluded in Table 14. Results of inductance are shown Figure 4.16: Simulation results for the differential inductance of dual layer inductors. It can be observed that results from the two simulators are very close. The difference is within $2 \%$, which proves the reliability of ASITIC.

Schematic of the proposed transformer is shown in Figure 4.15 and design parameters are concluded in Table 13. Simulation results are shown in Figure 4.17. At $2.4 \mathrm{GHz}$, the coupling factor is 0.53 .

\subsection{RC Network}

The $\mathrm{RC}$ filter in the proposed design is a low-pass filter. To reduce the magnitude and the bandwidth of the transfer function around demanding frequency range, large bias resistors of around $80 \mathrm{MOhm}$ are required. This is hard to realize on-chip; hence, the resistors are designed off-chip.

\subsection{Simulation}

Figure 4.18 shows the schematic for the proposed VCO design, including buffer. To simulate the transformer, inductors in the tank are simulated by connecting ideal inductors and series resistors. The resistance of the series resistors can be obtained from 


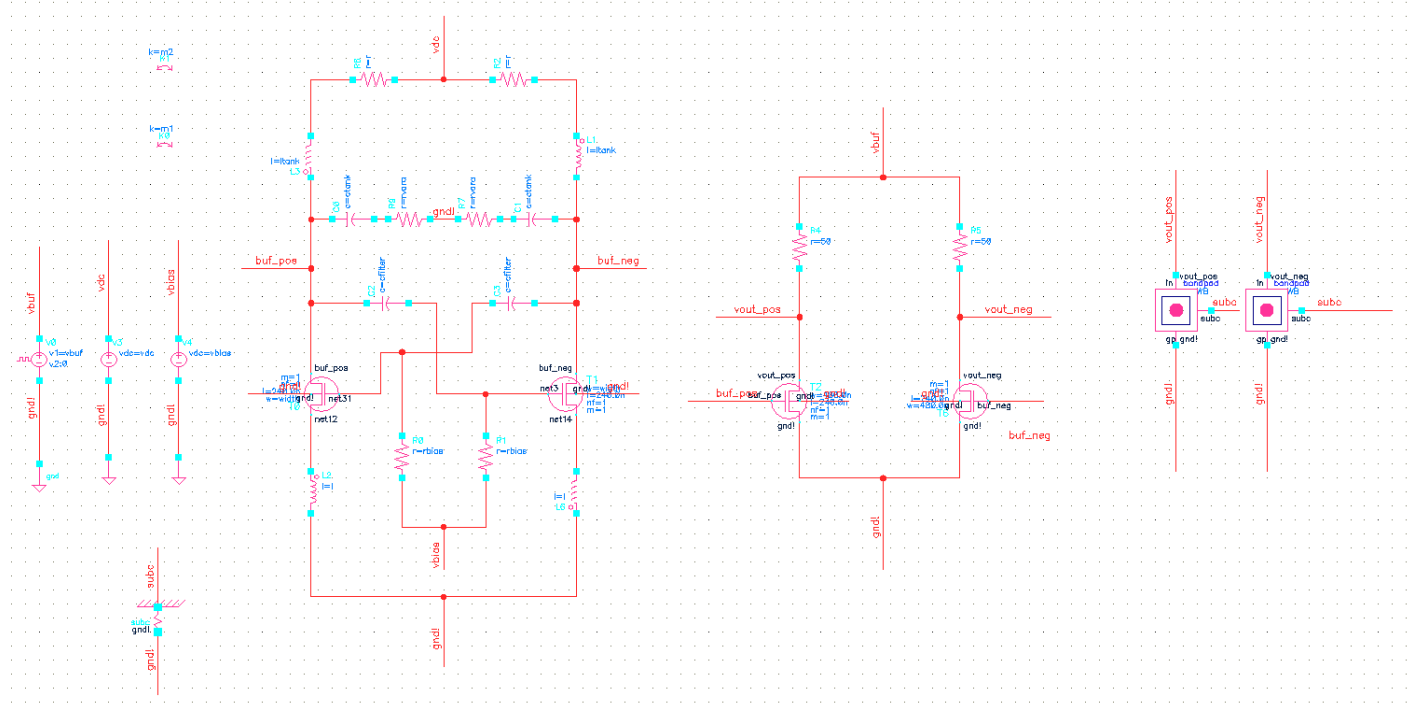

Figure 4.18: Schematic of final design, including buffer

Table 15: Design parameters

\begin{tabular}{c|c}
\hline Transistors for VCO Core & $12.6 \mathrm{um} / 120 \mathrm{~nm}$ \\
\hline Transistors for Buffer & $16 \mathrm{um} / 120 \mathrm{~nm}$ \\
\hline Supply Voltage (V) & 1 \\
\hline Bias Resistors (Ohm) & $80 \mathrm{M}$ \\
\hline Coupled Capacitors (F) & $2 \mathrm{p}$ \\
\hline Load Resistors for Buffer (ohm) & 50 \\
\hline Coupling Coefficient & 0.53 \\
\hline
\end{tabular}

Figure 4.4. To help with PSS analysis, varactors are also simulated by connecting capacitors and resistors in series. The value of series resistance can be obtained from Figure 4.8. The bias voltage is adjusted to get best phase noise performance at different operating frequency. Other design parameters are concluded in Table 15.

The transconductance of the VCO core transistor is $4.7 \mathrm{~mA} / \mathrm{V}$. The parallel resistance of the tank is about $630 \mathrm{ohm}$. According to the oscillation startup condition 
Table 16: Simualtion Results for proposed VCO

\begin{tabular}{c|c}
\hline Center Frequency (GHz) & 2.44 \\
\hline Tuning Range (\%) & 10 \\
\hline Phase Noise (dBc/Hz) & -132.8 \\
\hline $\begin{array}{c}\text { Power Consumption (mW) } \\
\text { (VCO core) }\end{array}$ & 3.01 \\
\hline Figure-of-Merit (dBc) & 195.8 \\
\hline
\end{tabular}

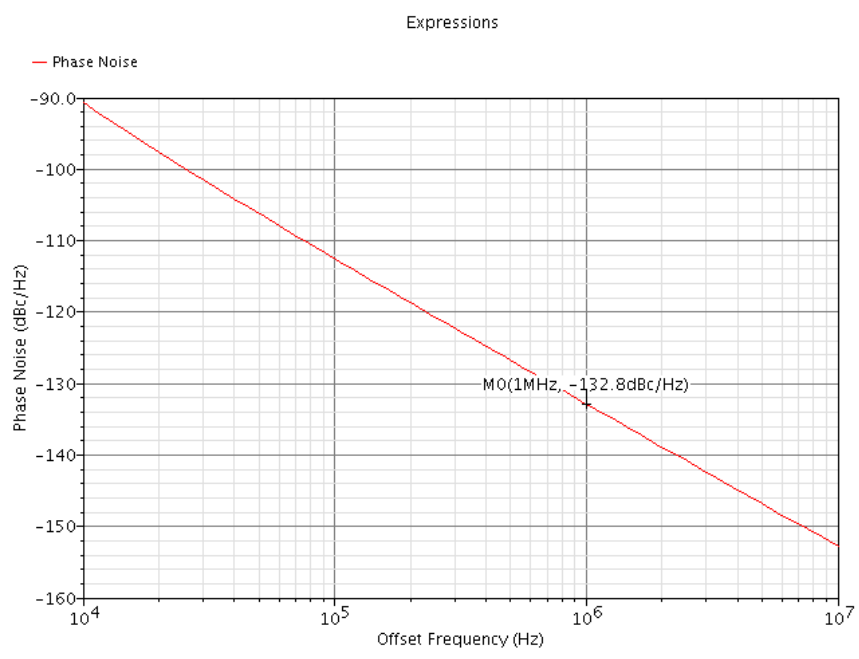

Figure 4.19: Simualtion result for the phase noise performance of the proposed VCO at $2.44 \mathrm{GHz}$

Table 17: Simulation results in different corners

\begin{tabular}{c|ccc}
\hline Corners & $\begin{array}{c}\text { Power Consumption } \\
(\mathbf{m W})\end{array}$ & $\begin{array}{c}\text { Phase Noise } \\
\mathbf{( d B c / H z})\end{array}$ & $\begin{array}{c}\text { FoM } \\
(\mathbf{d B c} / \mathbf{H z})\end{array}$ \\
\hline TT & 3.01 & -132.8 & 195.8 \\
SS & 2.73 & -131.6 & 195.0 \\
FF & 3.24 & -133.0 & 195.6 \\
SF & 2.77 & -132.4 & 195.8 \\
FS & 3.19 & -132.9 & 195.6 \\
\hline
\end{tabular}


Table 18: Comparison table for the simulation results

\begin{tabular}{c|c|c|c|c|c}
\hline Ref. & Tech & Freq $\mathbf{( G H z )}$ & $\begin{array}{c}\text { Phase Noise } \\
\mathbf{( d B c / H z})\end{array}$ & $\begin{array}{c}\text { Power } \\
\mathbf{( m W )}\end{array}$ & $\begin{array}{c}\text { FoM } \\
\mathbf{( d B c} / \mathbf{H z})\end{array}$ \\
\hline$[6]$ & $130 \mathrm{~nm}$ & $4.90-5.65$ & $<-130 @ 3 \mathrm{MHz}$ & 1.40 & 195.5 \\
\hline$[6]$ & $130 \mathrm{~nm}$ & $4.50-5.00$ & $-133 @ 3 \mathrm{MHz}$ & 1.40 & 196.0 \\
\hline$[21]$ & $180 \mathrm{~nm}$ & $6.09-7.50$ & $-123 @ 2 \mathrm{MHz}$ & 2.16 & 191.0 \\
\hline$[18]$ & $180 \mathrm{~nm}$ & $1.4 \pm 0$ & $-129 @ 1 \mathrm{MHz}$ & 1.46 & 190.0 \\
\hline$[18]$ & $180 \mathrm{~nm}$ & $3.8 \pm 4.2 \%$ & $-119 @ 1 \mathrm{MHz}$ & 0.57 & 193.0 \\
\hline This work & $130 \mathrm{~nm}$ & $2.44 \pm 5 \%$ & $-133 @ 1 \mathrm{MHz}$ & 3.07 & 195.8 \\
\hline
\end{tabular}

$$
g_{m}>\frac{2}{r_{p}}
$$

the circuit should start oscillating without problem.

Table 16 shows the simulation results of the VCO. Figure 4.19 shows the phase noise performance. Table 18 lists the comparison among this design with reported VCOs. Table 17 lists the phase noise, power consumption performance and FoM for different corners. Note that power consumed by the buffer is not included in the power consumption column.

\subsection{Conclusion}

This chapter has presented a circuit design based on the proposed topology. Simulation results show that this design shows good performance in either phase noise or power consumption, giving a more favorable figure of merit. Compared to the reported VCOs, this design achieves relatively high FoM. 


\section{Chapter: Chip Design and Evaluation}

\subsection{Introduction}

A chip based on the proposed circuit design in chapter 4 has been taped out using IBM $130 \mathrm{~nm}$ technology. Layout consideration is discussed first, followed by chip evaluation.

\subsection{Layout Consideration}

As has been pointed out in Chapter 4, the circuit is integrated on a single chip except for the bias resistors, which are too large to be implemented on the chip. The key to optimization is dynamic adjustment to linearize the circuit. Also, to reduce second harmonics, the layout is made as symmetric as possible. The final floor plan is shown in Figure 5.1.

\subsection{Evaluation}

\subsubsection{Equipment, Probes, and Station Setup}

To test the chip, two RF probes, one multicontact DC probe, and 2 DC needles are needed to land from the four cardinal directions. Important properties of the probes are highlighted in Table 19.

One spectrum analyzer and four voltage sources are needed for the testing. Since power consumption is one of the most important figures of the $\mathrm{VCO}$, high accuracy is in current consumption for VCO core is required. Accuracy of the power supply of the buffers and the tuning voltage of the varactors is less important. Accuracy of bias voltage is least important.

Table 20 summarizes the equipment used and its highlight properties.

Other tools needed include off-chip resistors, tweezers, glasses, instant glues, and cables. Testing station set up is shown in Figure 5.2. 


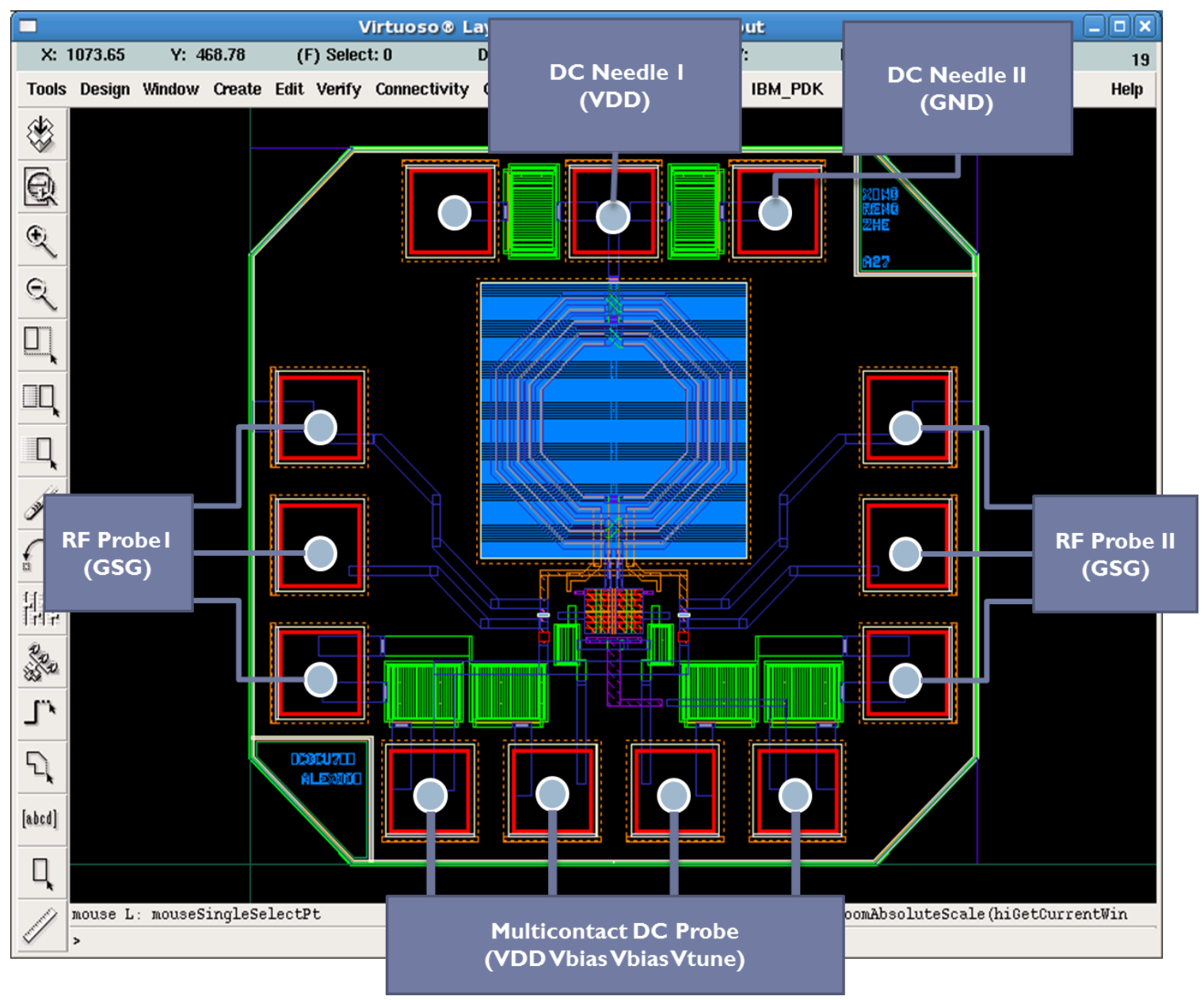

Figure 5.1: Floor plan for the chip.

Table 19: Table for probes

RF Probes (GSG) 40A-GSG-150-P

Quantity: 2

- $\mathrm{DC}$ to $40 \mathrm{GHz}$

- $\quad$ Pitch (tip spacing) $=150$ um

\section{Multicontact DC Probes MCW-22-8667}

Quantity: 1

- 4 Tips

- $\quad$ Pitch $=150$ um

DC Needles Quantity: 2


Table 20: Table for the testing equipment

\begin{tabular}{|c|c|}
\hline 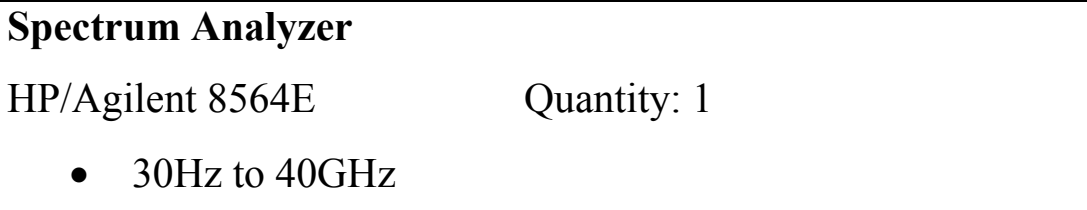 & 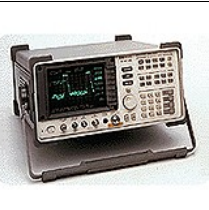 \\
\hline $\begin{array}{l}\text { DC Voltage Source I } \\
\text { Keithley } 2400 \text { Source Meter Quantity: } 2 \\
\quad \text { - } \quad 0.012 \% \text { basic measure accuracy with } 5 \frac{1}{2} \text {-digit resolution }\end{array}$ & $\begin{array}{r}0 \\
0 \\
0 \\
0 \\
\end{array}$ \\
\hline $\begin{array}{ll}\text { DC Voltage Source II/IV } & \\
\text { HP/Agilent E3646A } & \text { Quantity: } 1 \\
\text { Readback Accuracy } & \\
\text { Voltage: }<0.05 \%+5 \mathrm{mV} & \text { Current: }<0.15 \%+5 \mathrm{~mA}\end{array}$ & 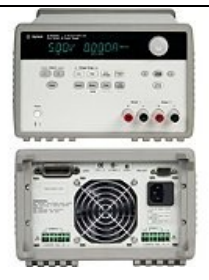 \\
\hline $\begin{array}{l}\text { DC Voltage Source III } \\
\text { HP/Agilent E3630A Quantity: } 1 \\
\begin{array}{l}\text { - } 10 \mathrm{mV}, 10 \mathrm{~mA} \text { resolution }\end{array}\end{array}$ & 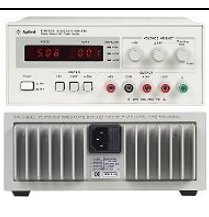 \\
\hline
\end{tabular}

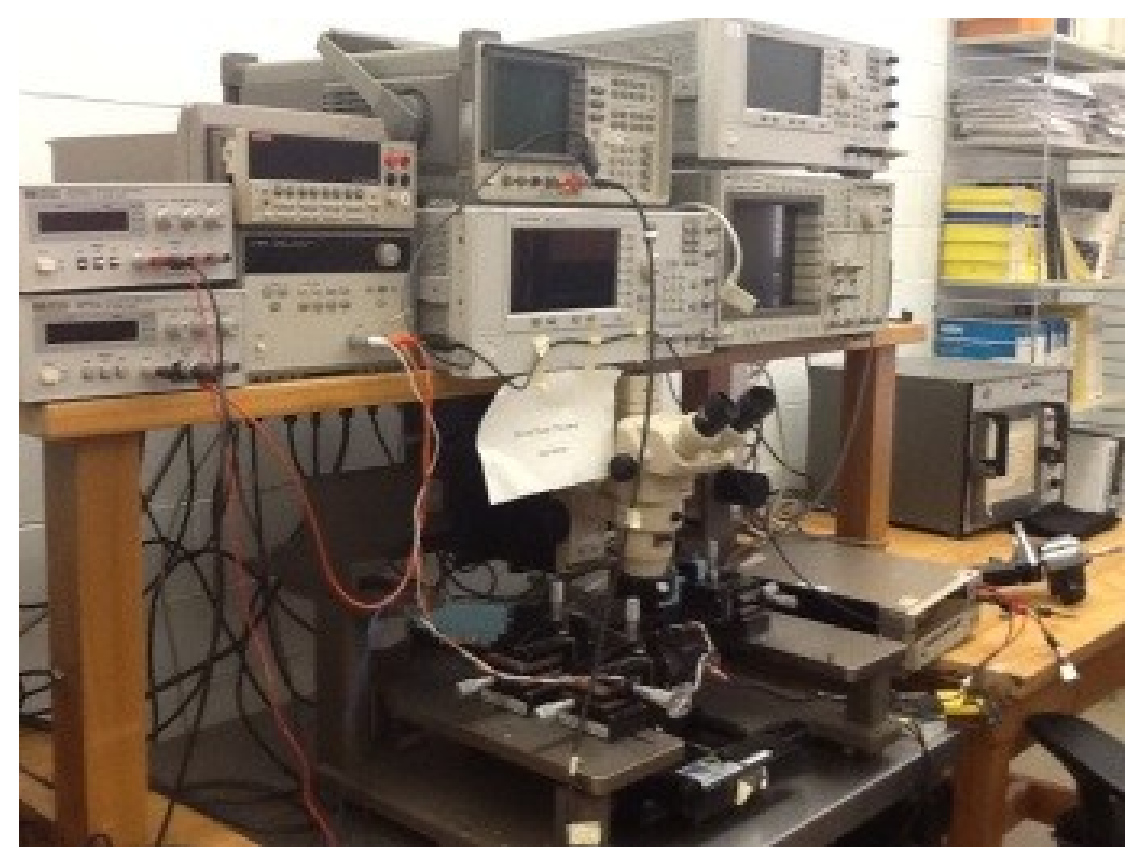

Figure 5.2: Testing station setup. 


\subsubsection{Measurement and Failure Analysis}

Several chips were tested with no output captured on the spectrum analyzer. This indicates an absence of AC signals in the circuit. This is often caused by a failure of startup in oscillation. Figure 5.3 is the test report for one of the tested chips. It turned out the buffers worked as expected, but not the VCO core.

The reasons for failure of oscillation startup can be complicated. One of the possible causes is the topology. The circuit can be simplified as Figure 5.4. Because the cross-coupled differential pair is blocked by two capacitors $C_{f i l t e r 1,2}$ from the $R C$ feedback network, no low frequency noise is accumulated within the positive feedback loop, which thus fails to start the oscillation. Therefore, an attempt to introduce disturbance by shorting the DC current in a very short time was carried out. However, no $\mathrm{AC}$ outputs were shown on the spectrum analyzer.

\subsubsection{Failure Analysis on the Quality Factor}

A possible reason for this failure could be over-estimation of the $Q$ of the tank. In this design, $Q$ of the tank varies from 17 to 18 over the oscillating frequency according to the simulation. However, in the real case, $Q$ can be decreased severely because of the unexpected parasitic resistance. The parasitic resistance will in turn increase energy consumption, thus larger positive feedback gain will be needed to start and keep the oscillation, namely larger $g_{m}$ of the active devices is required.

There are two methods to increase the positive feedback gain in testing. One of them is to increase the supply voltage manually. In this way, drain current $I_{d s}$ will be enlarged and $g_{m}$ will consequentially increase. Another method is to increase $V_{\text {bias }}$. This method will change the dc operating point, giving larger conduction angle of the transistors. Hence, more current will be allowed to inject into the tank. In this way the positive feedback gain is also increased.

In fact, the second method - increasing $V_{\text {bias }}$, is powerful to solve "the lack of positive feedback gain" issue. According to simulations, even with a very poor $\mathrm{Q}$, increasing $V_{\text {bias }}$ to suitable voltage may still start and maintain oscillation. Figure 5.5 (a)(c) show an case with $\mathrm{Q}=10$ with suitable $V_{\text {bias }}$. Note that other components in 


\section{Test Report}

Chip Number : $\underline{006}$

Testing Date: March $25^{\text {th }}, 2012$

Oscilation Test

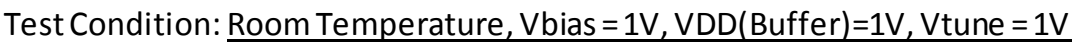

\begin{tabular}{|l|l|l|}
\hline VDD for VCO core $(\mathrm{V})$ & Range $(\mathrm{V})$ & Oscillation $(\mathrm{Y} / \mathrm{N})$ \\
\hline 0.8 & $0.799904-0.800096$ & $\mathrm{~N}$ \\
\hline 1 & $0.99988-1.00012$ & $\mathrm{~N}$ \\
\hline 1.4 & $1.399832-1.400168$ & $\mathrm{~N}$ \\
\hline 1.6 & $1.599808-1.600192$ & $\mathrm{~N}$ \\
\hline 2 & $1.99976-2.00024$ & $\mathrm{~N}$ \\
\hline
\end{tabular}

Note: No output on spectrum analyzer under $0.8-2 \mathrm{~V}$.

\section{Current Comsuption for VCO Core Test}

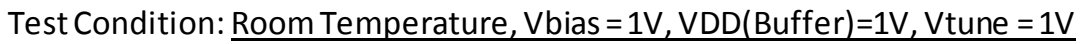

\begin{tabular}{|l|l|l|}
\hline VDD for VCO core $(\mathrm{V})$ & Range $(\mathrm{V})$ & Current Consumption $(\mathrm{mA})$ \\
\hline 0.8 & $0.799904-0.800096$ & 0.2468 \\
\hline 1 & $0.99988-1.00012$ & 0.3652 \\
\hline 1.4 & $1.399832-1.400168$ & 1.2516 \\
\hline 1.6 & $1.599808-1.600192$ & 2.0493 \\
\hline 2 & $1.99976-2.00024$ & 2.7852 \\
\hline
\end{tabular}

Note: $\underline{N} / \mathrm{A}$

\section{Current Comsuption for VCO Buffer Test}

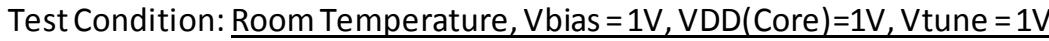

\begin{tabular}{|l|l|l|}
\hline VDD forVCO core $(\mathrm{V})$ & Range $(\mathrm{V})$ & Current Consumption $(\mathrm{mA})$ \\
\hline 0.6 & $0.5947-0.6053$ & 5 \\
\hline 0.8 & $0.99988-1.00012$ & 6 \\
\hline 1 & $1.399832-1.400168$ & 9 \\
\hline 1.2 & $1.599808-1.600192$ & 10 \\
\hline 1.4 & $1.99976-2.00024$ & 12 \\
\hline
\end{tabular}

Figure 5.3: One test report example. 


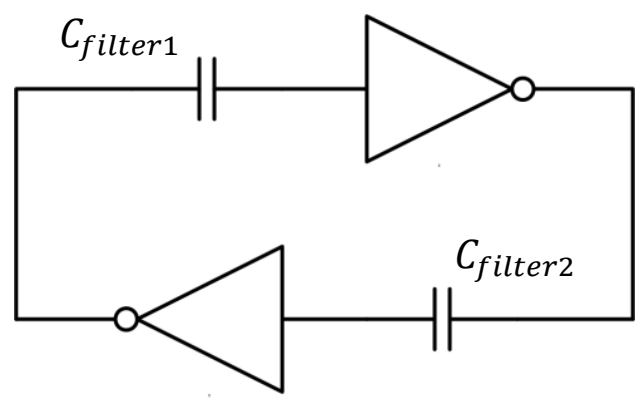

Figure 5.4: Simplified diagram for the proposed VCO.

Expressions

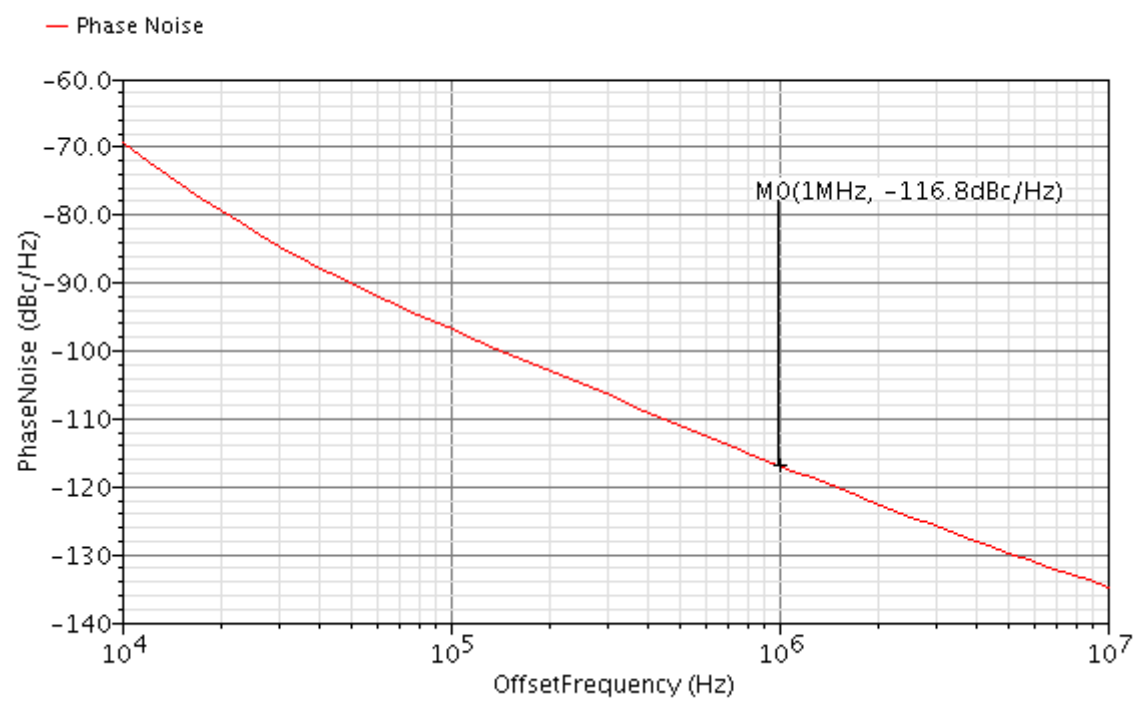

(a) 


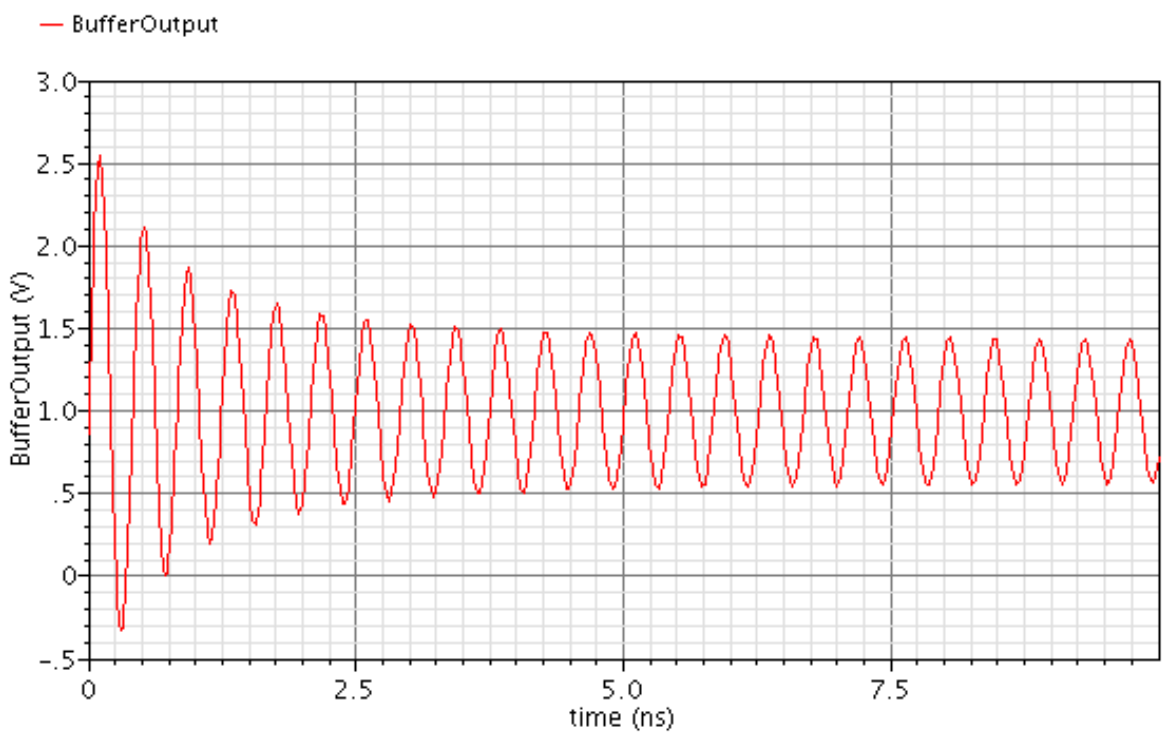

(b)

Transient Response

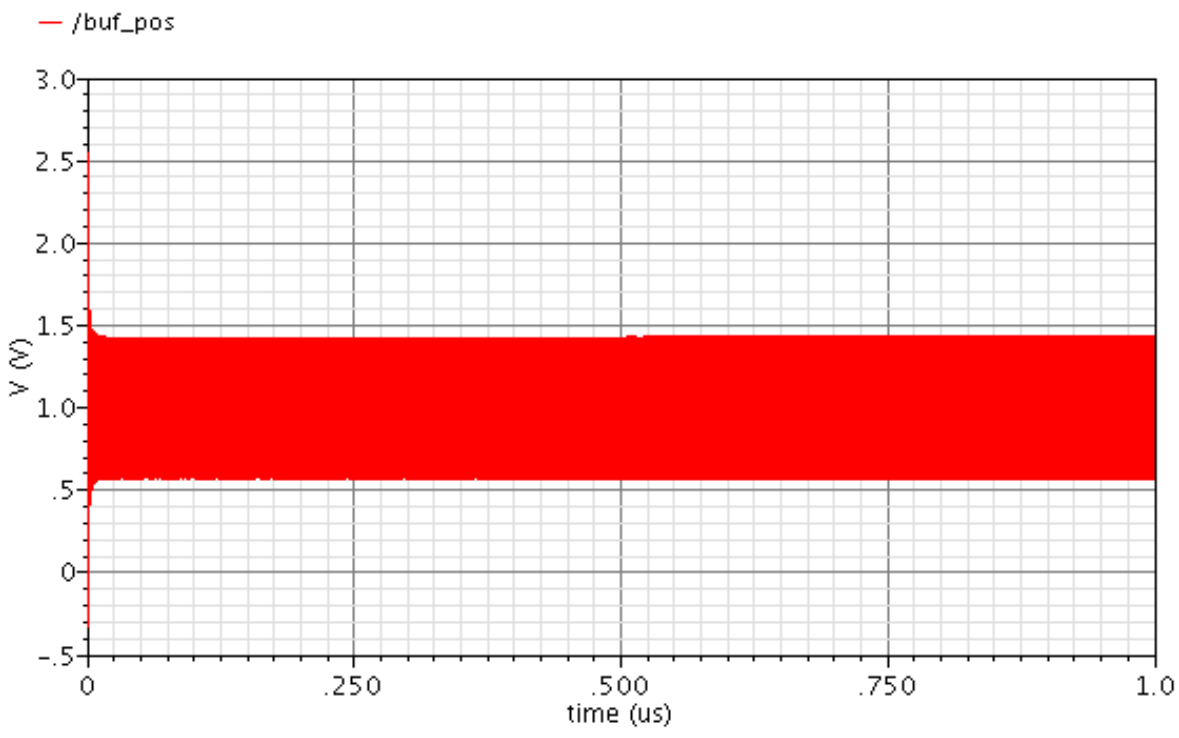

(c)

Figure 5.5: (a) Phase Noise (b) Transient response (0-10ns) (c) Transient response (0-1us) for $\operatorname{tank} Q=10$. 
the circuit remain the same. It turned out when $V_{\text {bias }}$ is increased to $0.5 \mathrm{~V}$, despites the phase noise performance (see Figure 5.5(a)) is lower, the VCO is still able to work properly (see Figure 5.5 (b)(c)).

During the chip testing both of these two methods were carried out. At the same time, shorting the DC current in very short time was also tried under higher power supply or higher $V_{\text {bias }}$ to help start oscillation. However, still no AC output showed up on the spectrum analyzer. This indicates the failure of oscillation is not caused by the reduction of $Q$.

\subsubsection{Failure Analysis on the Transformer}

Another possible reason could be that the coupling coefficient is not estimated appropriately. In the design, the coupling coefficient is estimated to be 0.53 . However in reality the value of coupling coefficient may be much lower than expected. Hence, simulations have been carried out to verify how different coupling coefficient affects oscillation.

To simulate the worst case, coupling coefficient is set to -0.7 . Other conditions are kept the same as the design. Not surprisingly, the oscillation died out after tens of nanoseconds (see Figure 5.6).

However, during the simulating, it turned out through increasing $V_{\text {bias }}$, the oscillation can be maintained. When $V_{\text {bias }}$ is increased to $0.5 \mathrm{~V}$, the VCO is able to function although the phase noise performance and power consumption becomes worse (see Figure 5.7 (a)-(c)).

As mentioned in the previous analysis, $V_{\text {bias }}$ was increased during testing. However still no AC output shown on the spectrum analyzer. This indicates the failure is not caused by the inaccurate estimation of transformer's coupling coefficient.

\subsubsection{Failure Analysis on Pad Capacitance}

Another possible reason comes from the underestimation of the capacitance of the pads. 


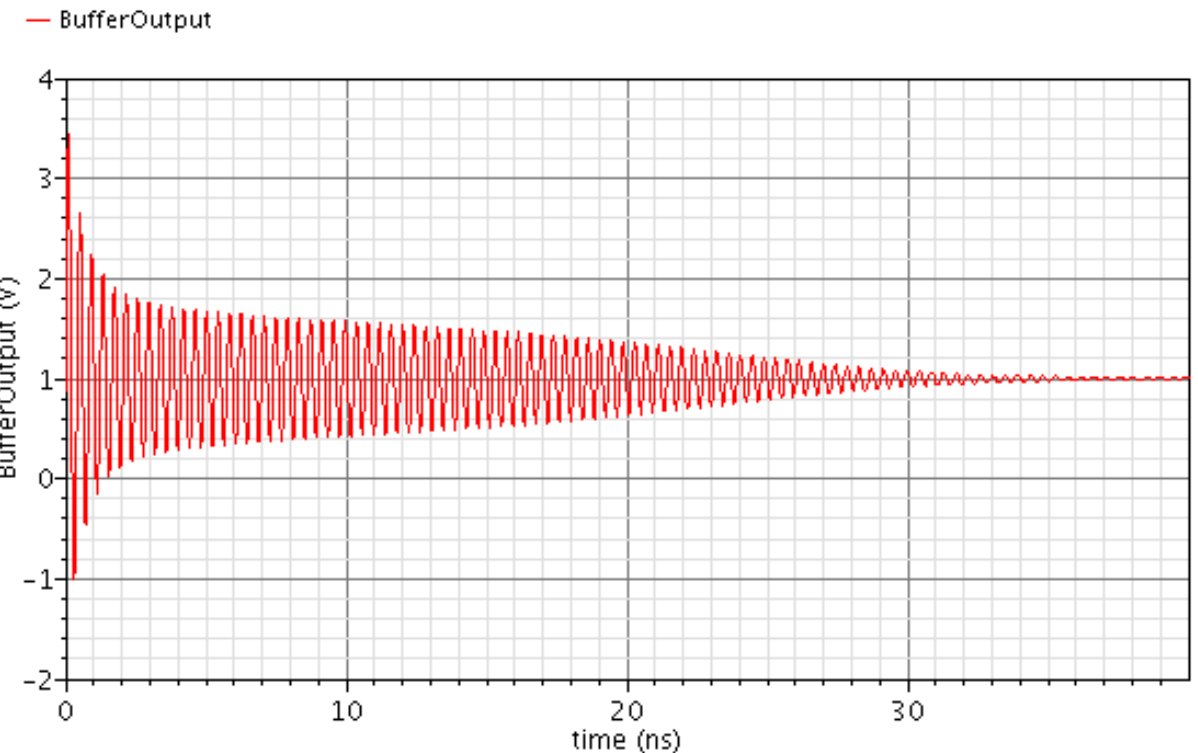

Figure 5.6: Transient response for $k=0.01$.

Expressions

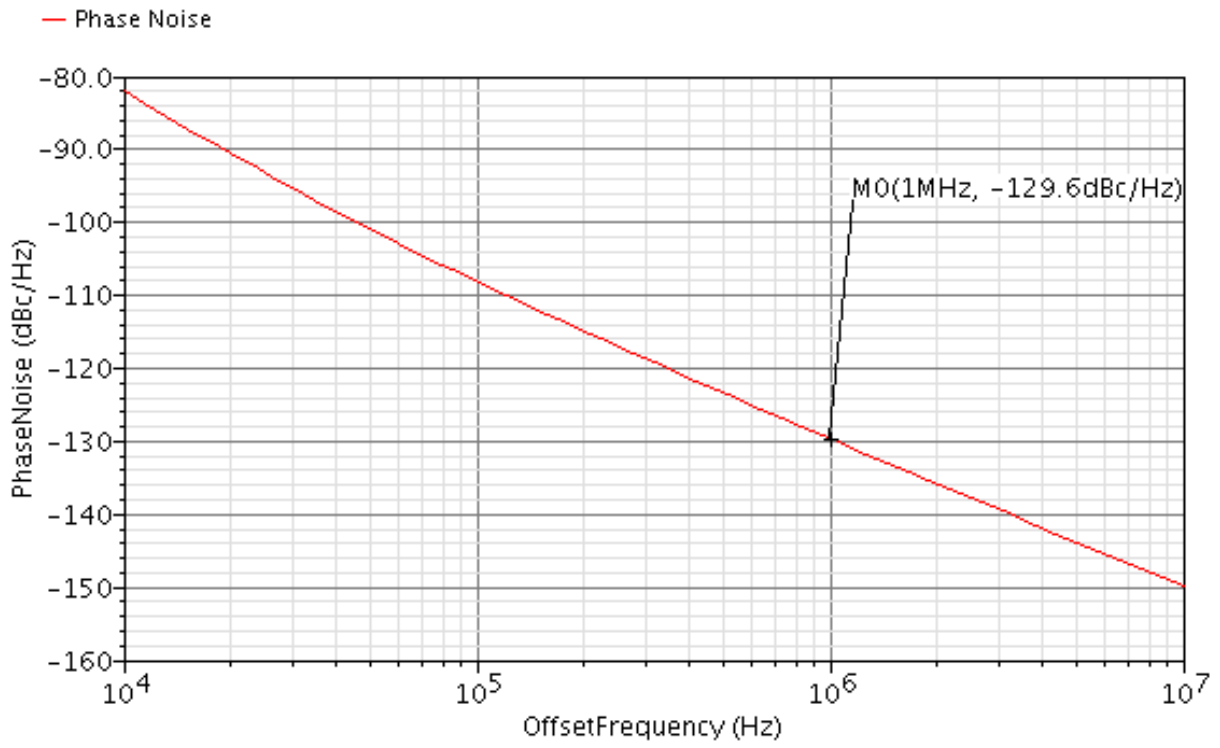

(a) 
- Bufferoutput

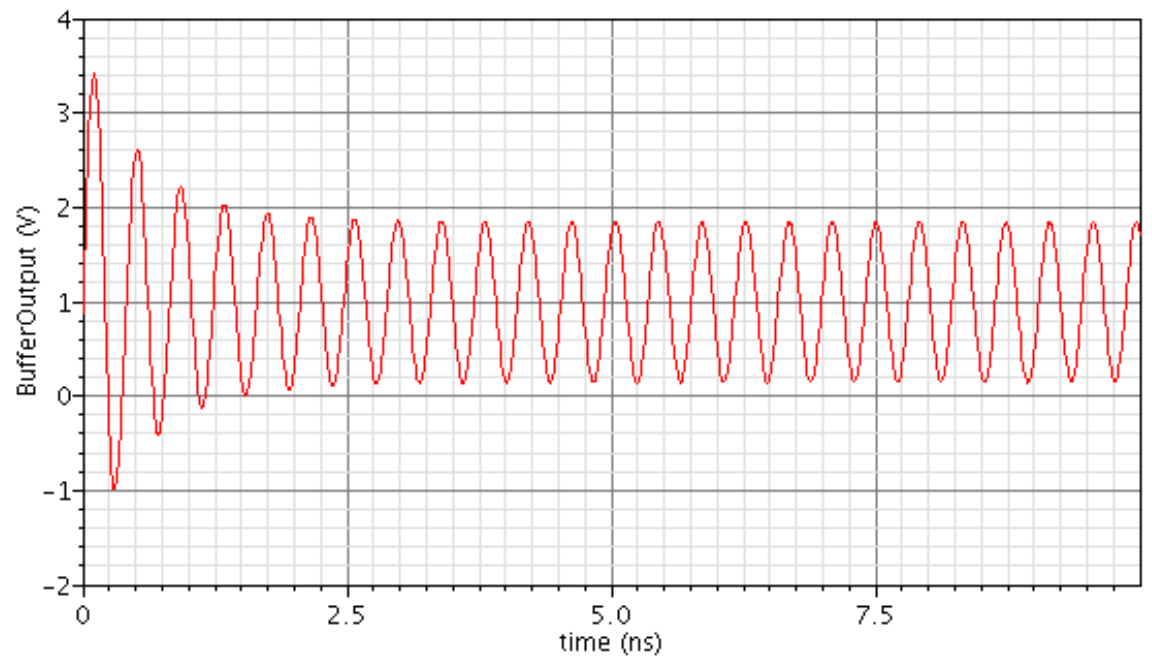

(b)

Transient Response

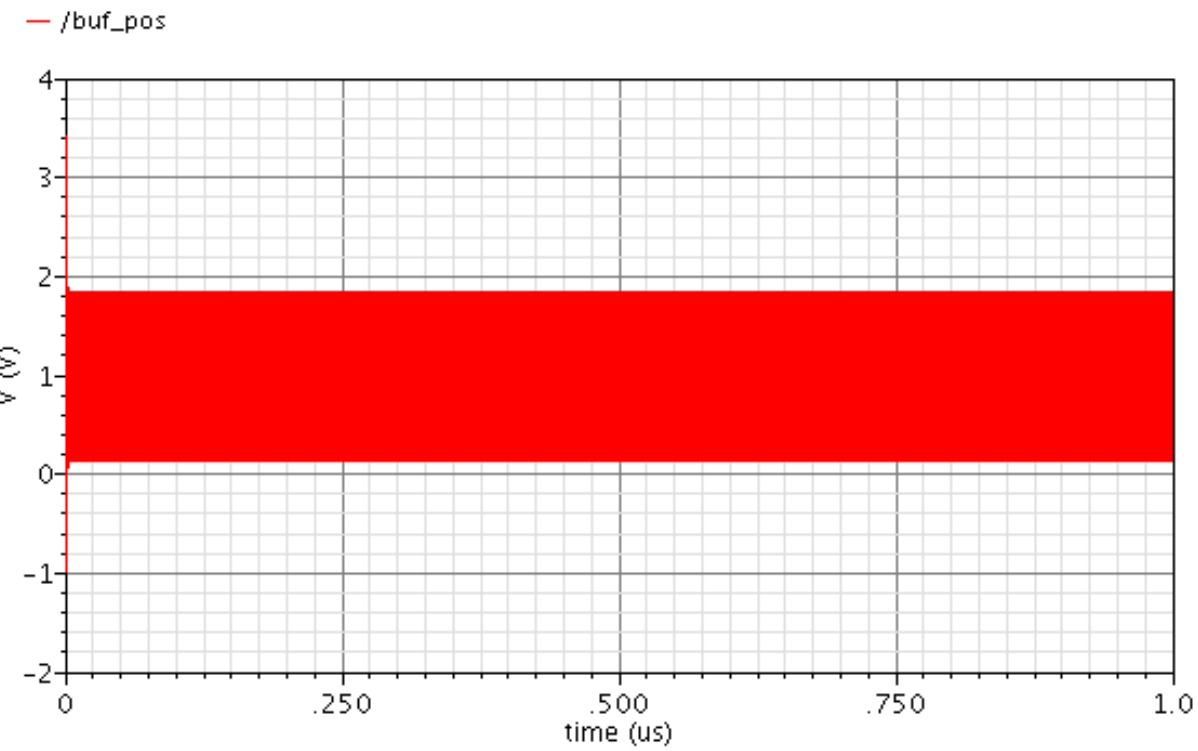

(c)

Figure 5.7: (a) Phase Noise (b) Transient response (0-10ns) (c) Transient response (0-1 us) for $\mathrm{k}=0.01$ with $V_{\text {bias }}=0.5 \mathrm{~V}$. 


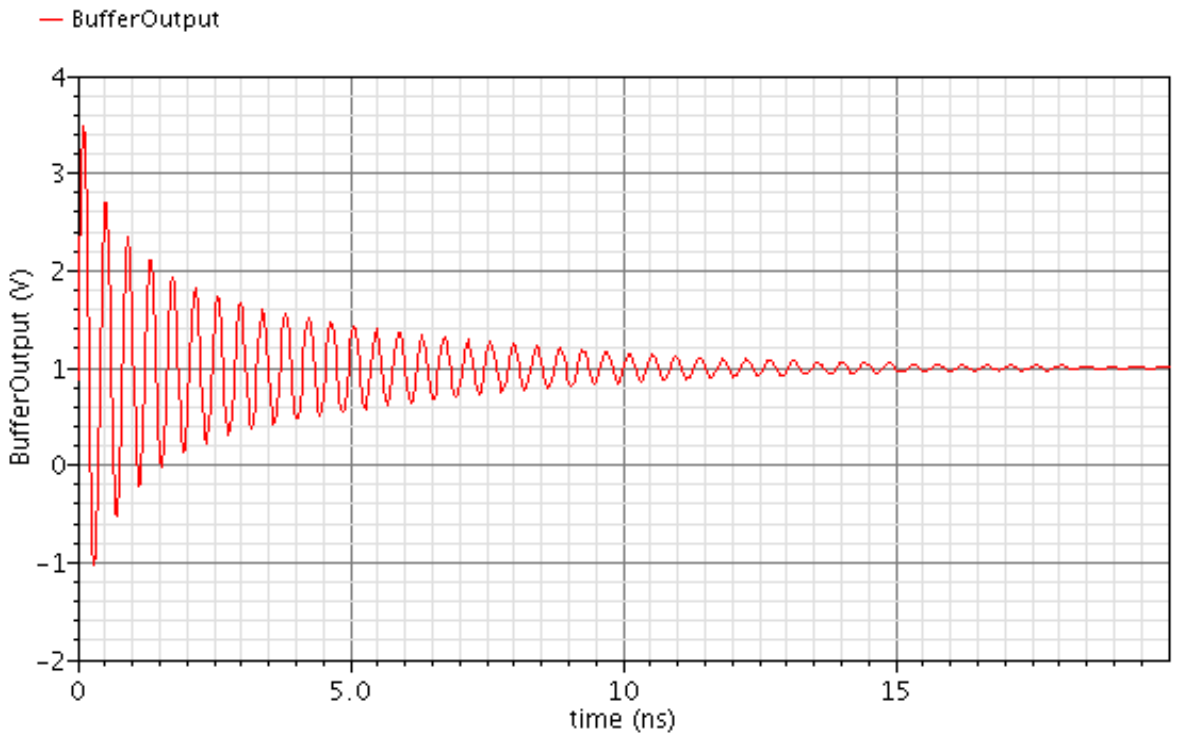

Figure 5.8: Transient response for VCO with extra pad capacitance.

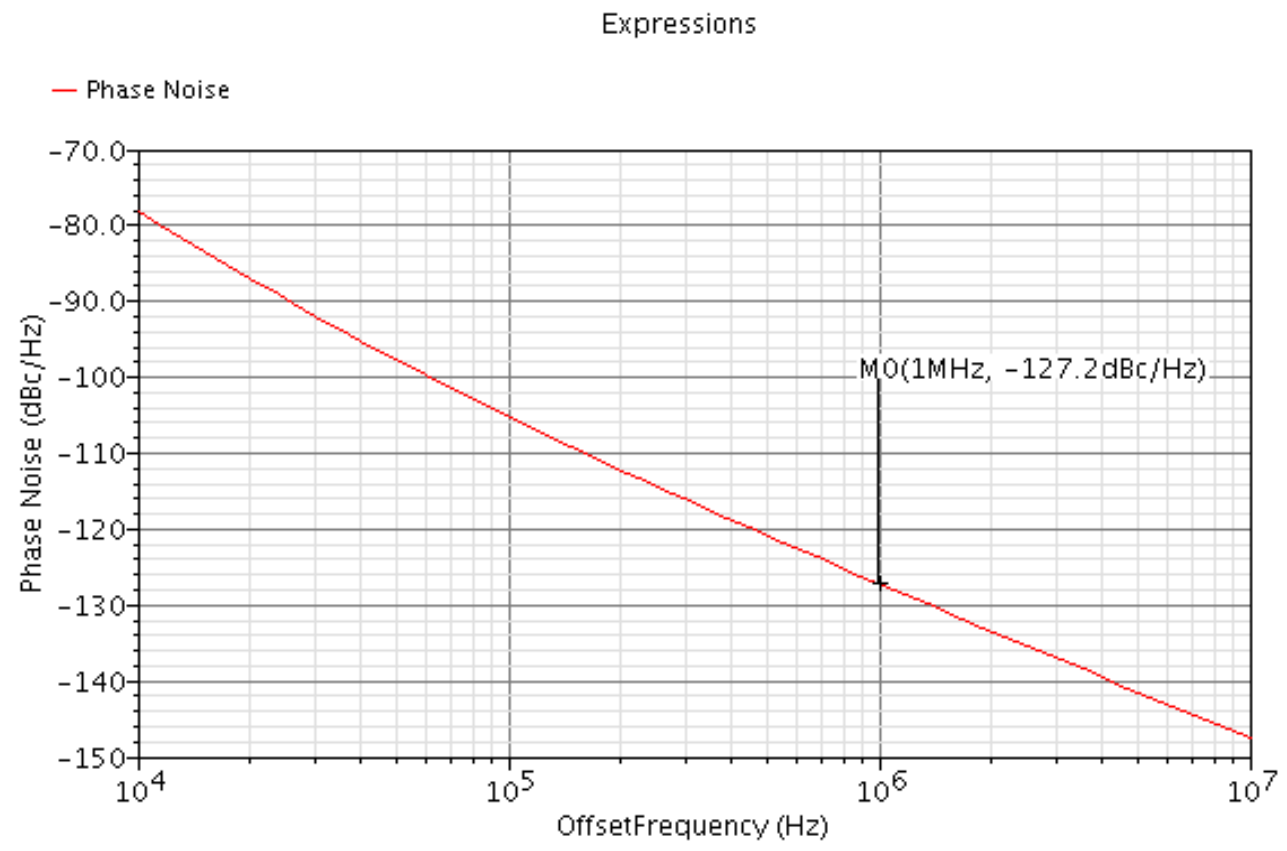

(a) 
- Bufferoutput

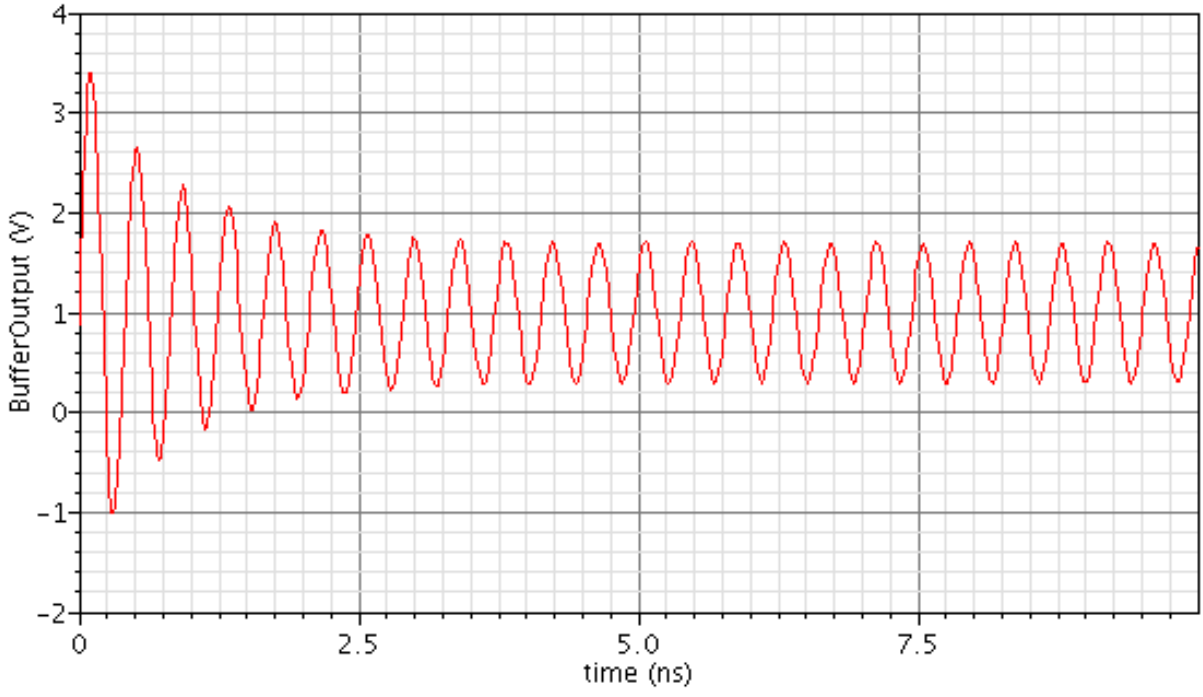

(b)

Transient Response

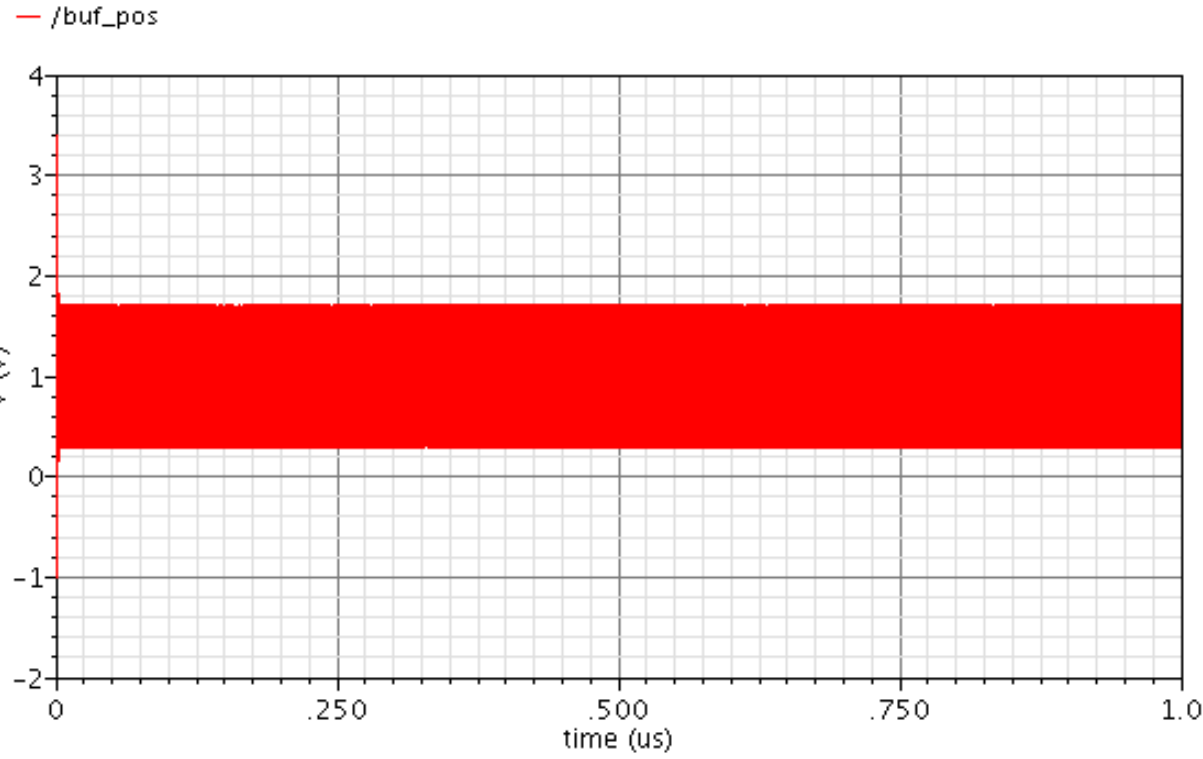

(c)

Figure 5.9: (a) Phase Noise (b) Transient response (0-10ns) (c) Transient response (0-1us) for VCO with extra pad capacitance when $V_{\text {bias }}=0.5 \mathrm{~V}$. 
The extra parasitic capacitance of the pads may cause the VCO fail to drive the loads and consequentially die out.

To simulate the worst case, two extra $2 \mathrm{pF}$ capacitors are added onto the differential outputs of the buffer in parallel with the pads. Similar to the previous cases, the oscillation died out when all the parameters are kept same as the design (see Figure 5.8). However in simulation when $V_{\text {bias }}$ is increased, the VCO works (see Figure 5.9). Hence the underestimation of the capacitance of the pads is not the reason to cause the startup failure.

\subsubsection{Cause of Failure}

After investigating the possibilities above, the layout view has been checked. Eventually, it is found that the reason to cause the failure of the oscillation is the misconnection between the primary inductors of the transformer and the varactor array of the circuit. Because of the transformer is designed by the author, the transformer is not included in the LVS verification, as well as its connection to the rest part of the circuit.

The connection part between the primary inductor and the varactor array is realized by stacking metal layer MQ, MG and LY appropriately to the top two layers. This strategy is to reduce the parasitic resistance as much as possible, aiming to maintain a high quality factor of the tank.

Figure 5.10 shows a varactor array using 8 differential varactor units. The drain sides are connected using metal layer M3. Then metal layer MQ is used to connect the differential gates. Figure 5.11 shows how three varactor arrays are connected using metal MQ. Then such pattern is repeated. Figure 5.12 shows the layer MQ in the whole varactor array $(32 * 15$ units), other layers are turned off to make the view clear. Then the odd and even number metal bars shown in Figure 5.12 are connected together by metal MG respectively. Then MG is connected to the top layer by stacking the metal layers.

However, in the connection part between the primary inductors and the varactor array, two extra pieces of MQ metal are added by mistake (see Figure 5.13, one of the 


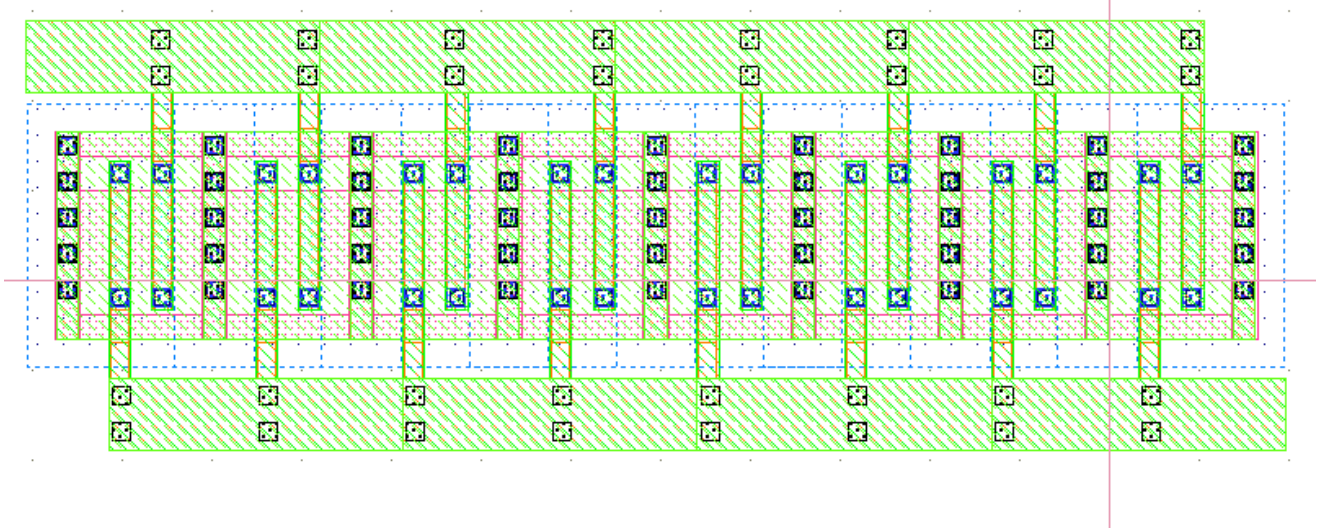

Figure 5.10: Layout view for 8*1 varactor array

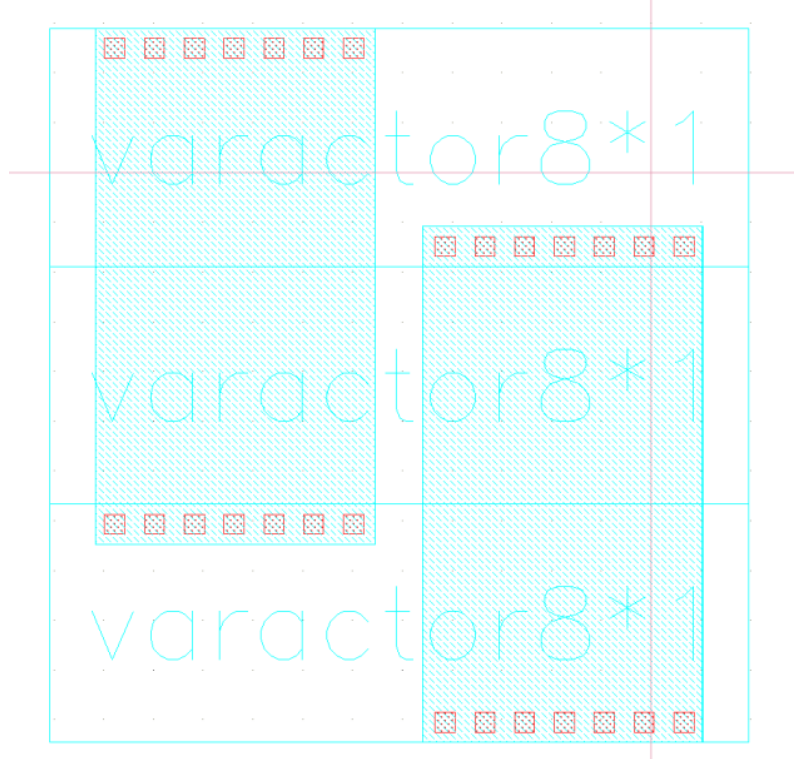

Figure 5.11: Layout view for $8 * 3$ varactor array

unwanted metal is highlighted as an example), resulting in connecting all the gates of the differential varactor array together. The tank is then shorted as shown in Figure 5.14. 


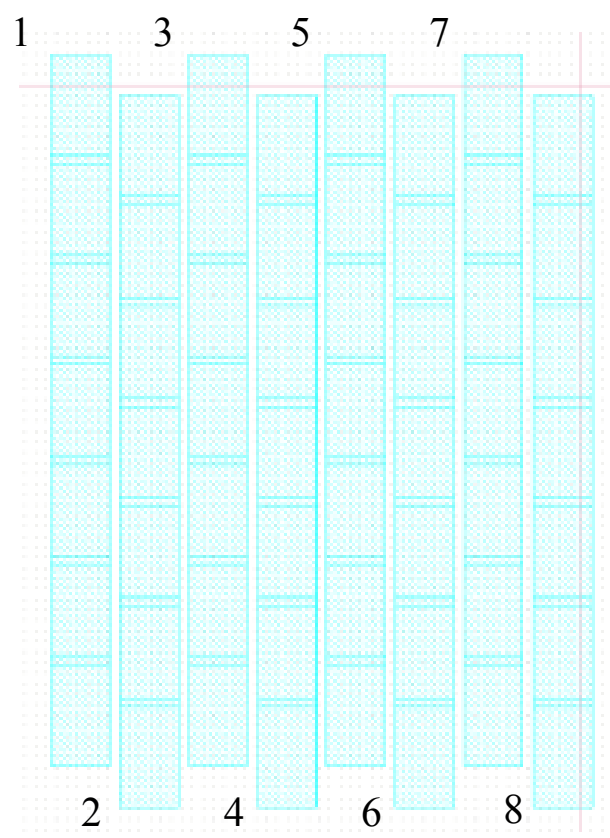

Figure 5.12: Expecting layer MQ for the entire varactor array (32*15 units)

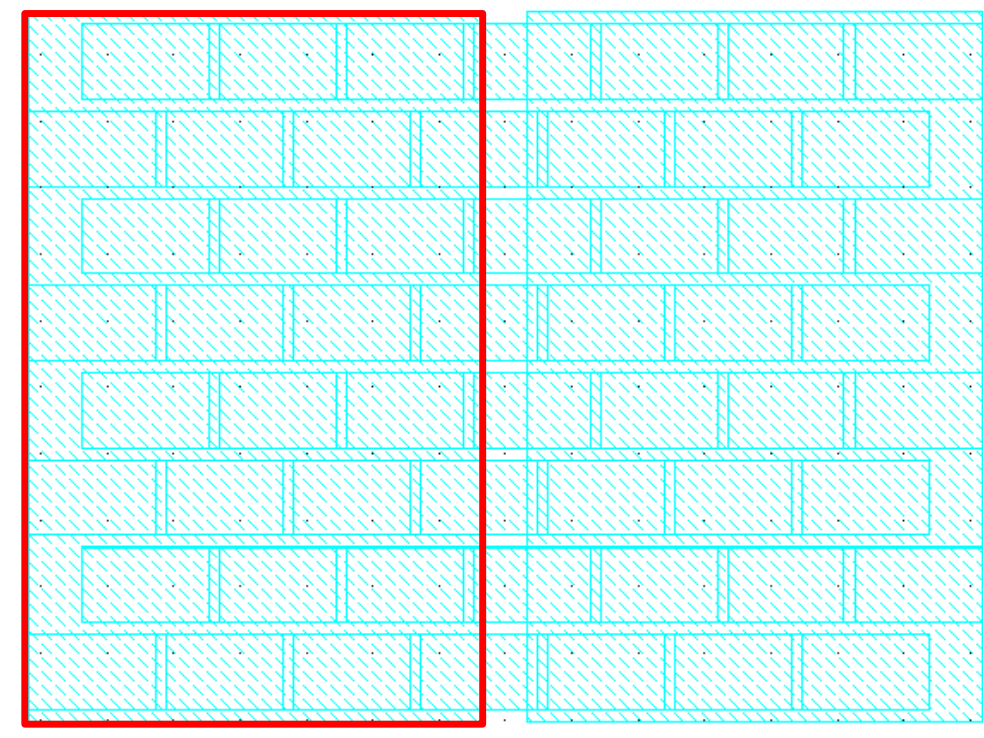

Figure 5.13: Wrong layout view for layer MQ 


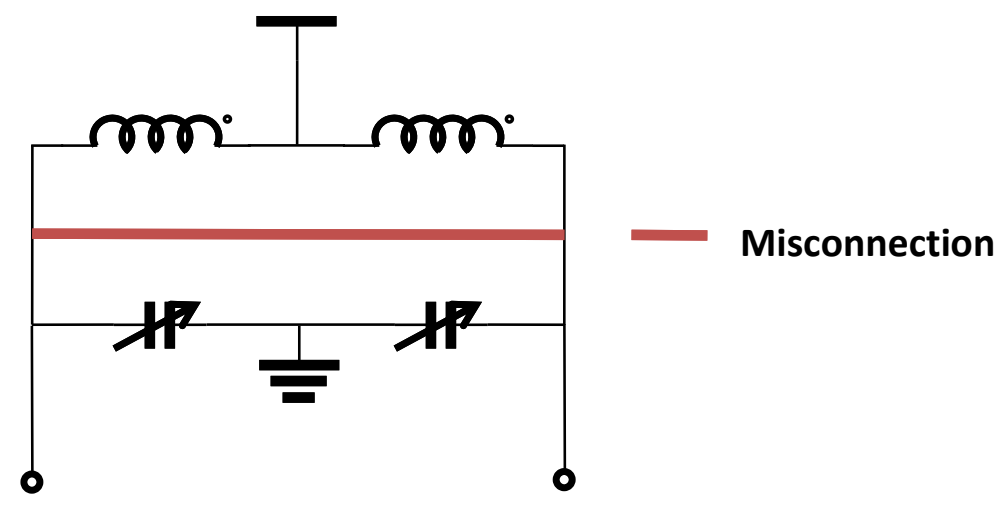

Figure 5.14: Equivalent circuit for the misconnected tank

This misconnection will make the circuit fail to oscillate no matter what adjustment in the supply voltage or bias voltage has been made. This is in accordant with the observation in the testing. Also, this misconnection is equivalent to connect the gates of the buffer amplifiers to the DC power supply (1V) directly since there is no ac signal in the tank, which also matches the testing results in the measurement.

\subsection{Conclusion}

This chapter presents the chip design and evaluation. Layout consideration is briefly discussed first, followed by the display on the measurement setup.

The chip did not work as expected. It failed to start oscillation. To find the problem, failure analysis has been made to find out the reason that caused the oscillation failure. After exploring the possible reasons, including tank, transformer and load capacitance, it is found the reason that causes the failure of the oscillation is the misconnection between the primary inductors and the varactor array. 


\section{Chapter: Conclusion}

\subsection{Conclusion of the Thesis}

This thesis researches the relation between phase noise and non-linear behavior. In Chapter 2, previous researches on VCO theory, including both linear and non-linear approaches, have been reviewed first. Circuit examples are then reviewed with analysis.

In Chapter 3, an attempt to link the non-linear behavior to the phase noise has been made starting with a hypothesis that the phase noise is determined by the harmonic components of the circuit. Simulation results proved a definite link between the phase noise and the harmonic components. At the same time, simulation results also indicated the existence of other factors. Hence another assumption, that phase noise is determined by the transfer function and harmonics, has been put forward to explain the generation of phase noise. With this insight, harmonic content can be used as the design optimization tool in search of an optimum FoM topology. A VCO circuit using harmonic and transfer function suppression technique is then proposed to decrease phase noise. Simulations results show that the circuit has less harmonics and narrower transfer function with lower peak amplitude. The circuit provides 10dB improvement phase noise performance compared to the circuit without employing harmonics suppression and transfer function reduction techniques.

In Chapter 4, a $2.4 \mathrm{GHz}$ VCO using the proposed topology has been designed with IBM 130nm technology. Design issues, including devices selection (inductors, varactors, transformer, etc), circuit optimization and layout consideration have been discussed first, followed by simulations. Results show that the phase noise performance and FoM of this design is among the state of arts.

Chapter 5 presented the chip evaluation and failure analysis process.

Measurement set up is shown at first. Measurement results indicated that the chip is 
failed to start up oscillating. Then several possible reasons are listed and discussed. Finally the reason that fails the oscillation is the misconnection between the primary inductors and the varactor array.

\subsection{Conclusion of the Contribution}

The contribution of this thesis is an exploration of the relation between non-linear behavior and phase noise. Although people may have been aware of the relation between harmonics and phase noise, studies on examining this relation have not been reported.

\subsection{Future Work}

This thesis showed an attempt to link the phase noise performance with transfer function and harmonic components. Although the reported research proved the hypothesis, evidences are still demanded to make it general.

Another topic is to expand work on the VCO circuit topologies using the proposed VCO design guideline. 


\section{References}

[1] E.Hegazi, "A filtering technique to lower LC oscillator phase noise," IEEE Journal of Solid-State Circuits, vol. 36, no. 12, pp. 1921-1930, Dec. 2001.

[2] B. Razavi, Monolithic Phase-Locked Loops and CLock Recovery circuits: theory and design., New Jersey: IEEE Press, 1996.

[3] D. Wolaver, Phase-Locked Loop circuit deisgn, New Jersy: Prentice-Hall Inc, 1991.

[4] K. Kouznetsov, "Phase Noise in LC Oscillators," IEEE Journal of solid state circuits, vol. 33, no. 2, pp. 179-194, 1998.

[5] M. Tiebout, Low power VCO design in CMOS, Springner, 2006.

[6] A.Mazzanti, "Clacc-C harmonic CMOS VCOs, with a general result on phase noise," IEEE Journal of Solid-State Circuits, vol. 43, no. 12, pp. 2716-2729, Dec. 2008.

[7] P. Andreani, "A 2.3GHz LC-tank CMOS VCO with optimal phase noise performance," in ISSCC, Feb. 2006.

[8] P. Andreani, "A study of phase noise in colpitts and LC-tank CMOS oscillators," IEEE Journal of Solid-State circuits, vol. 40, no. 5, pp. 1107-1118, May 2005.

[9] P. Andreani, "Phase noise analysis of the LC-Tank CMOS Oscillator," in Norchip Conference, Nov. 2004.

[10] J. Chen, "A current shaping technique to lower phase noise in LC oscillators," in ICECS 2008, Aug. 2008. 
[11] J. Chen, "A low power, startup ensured and constant amplitude class-c VCO in 0.18um CMOS," IEEE Microwave and Wireless Components Letters, vol. 21, no. 8, pp. 427-429, Aug. 2011.

[12] W. Deng, "A feedback class-C VCO with robust startup condition over PVT variations and enhanced oscillation swing," in ESSCIRC, Helsinki, Sept. 2011.

[13] A. Hajimiri, "A general theory of phase noise in electrical oscillators," IEEE Journal of Solid-State Circuits, vol. 33, no. 2, p. 179194, Feb. 1998.

[14] A. Fard, "An analysis of 1/f2 phase noise in bipolar colpitts oscillators (with a digression on bipolar differential-pair LC oscillators)," IEEE Journal of Solid-State Circuits, vol. 42, no. 2, pp. 374-384, Feb. 2007.

[15] A. Hajimiri, "Design issues in CMOS differential LC oscillators," IEEE Journal of Solid-state Circuits, vol. 34, no. 5, pp. 717-724, May 1999.

[16] D. Ham, "Virtual damping and einstein relation in oscillators," IEEE Journal of Solid-State Circuits, vol. 38, no. 3, pp. 407-418, Mar. 2003.

[17] Q. Huang, "Phase noise to carrier ratio in LC oscillators," IEEE Transactions Circuits and Systems-I:Fundamental Theory and Applications, vol. 47, no. 7, pp. 965-980, Jul. 2000.

[18] K. Kwok, "Ultra-low-voltage high-performance CMOS VCOs using transformer feedback," IEEE Journal of Solid-State Circuits, vol. 40, no. 3, pp. 652-660, Mar. 2005.

[19] T. Lee, "Oscillator phase noise: a tutorial," IEEE Journal of Solid-State Circuits, vol. 35, no. 3, pp. 326-336, Mar. 2000. 
[20] A. Mazzanti, "A 1.4mW 4.90-to-5.65GHz Class-C CMOS VCO with an Average FOM of $194.5 \mathrm{dBc} / \mathrm{Hz}, "$ in $I S S C C$, Feb. 2008.

[21] A. Mazzanti, "A push-pull class-c CMOS VCO," IEEE Journal of Solid-State Circuits, vol. 48, no. 3, pp. 724-733, Mar. 2013.

[22] D. Murphy, "Phase noise in LC oscillators: a phasor-based analysis of a general result and of loaded Q," IEEE Transactions on Circuits and Systems-I: Regular Papers, vol. 57, no. 6, pp. 1187-1203, Jun. 2010.

[23] K. Okada, "A 0.114-mW dual conduction class-c CMOS VCO with 0.2-V power supply," VLSI circuits, pp. 228-229, Jun. 2009.

[24] R.Aparicio, "A noise-shifting differential colpitts VCO," IEEE Journal of SolidState Circuits, vol. 37, no. 12, pp. 1728-1737, Dec. 2002.

[25] J. Rael, "Physical processes of phase noise in differential LC oscillators," in CICC, May 2000.

[26] B. Soltanian, "Tail current-shaping to improve phase noise in LC voltage-controlled oscillators," IEEE Journal of Solid-State Circuits, vol. 41, no. 8, pp. 1792-1802, Aug. 2006.

[27] D. Leeson, "A simple model of feedback oscillator noise spectrm," in IEEE Proceeding, Feb. 1966.

[28] H. Barkhausen, Elektronen Rohren, III, Leipzig: Hirzel, 1935.

[29] H. Darabi, "Noise in CMOS mixers: a simple physical model," IEEE Journal of Solid-State Circuits, vol. 35, no. 1, Jan. 2000.

[30] C. Park, "A low-noise, 900MHz VCO in 0.6um CMOS," IEEE Journal of Solid- 
State Circuits, vol. 34, no. 5, pp. 586-591, 1999.

[31] B. Razavi, "A study of phase noise in CMOS oscillators," IEEE Journal of SolidState Circuits, vol. 31, pp. 331-343, Mar. 1996.

[32] A. Enam, "A 300MHz CMOS Voltage-Controlled ring oscillator," IEEE Journal of Solid-State Circuits, vol. 25, no. 1, pp. 312-315, Feb. 1990.

[33] A. Bonfanti, "Flicker Noise Up-Conversion due to Harmonic Distortion in Van der Pol CMOS Oscillators," IEEE TRANSACTIONS ON CIRCUITS AND SYSTEMS, vol. 59, no. 7, pp. 1418-1430, 2012.

[34] N. Tchamov, "Technique for flicker noise up-conversion suppression in differential LC oscillators," IEEE Transactions on circuits and systems, vol. 54, no. 11, pp. 959963, Nov. 2007.

[35] J. Groszkowski, "The interdependence of frequency variation and harmonic content, and the problem of constant-frequency oscillators," Proceedings of IRE, vol. 21, pp. 958-981, Jul. 1933.

[36] "On the Bias Noise to Phase Noise Conversion in Harmonic Oscillators Using Groszkowski Theory," in 2011 IEEE International Symposium on ISCAS, Rio de Janeiro, May 2011.

[37] A. Papoulis, Probablity, random viariables and stochastic process, New York: Tata McGraw-Hill, 2002.

[38] C. Yue, "Physical modeling of spiral inductors on silicon," IEEE Transactions on electron devices, vol. 47, no. 3, pp. 560-568, Mar. 2000.

[39] S. Mohan, "Simple Accurate Expressions for Planar Spiral Inductances," IEEE 
Journal OF Solid-States Circuits, vol. 34, no. 10, pp. 1419-1424, Oct. 1999.

[40] Y. Cao, "Frequency-independent equivalent-circuit model for on-chip spiral inductors," IEEE Journal of Solid-State Circuits, vol. 38, no. 3, p. 419426, Mar. 2003.

[41] J. Burghartz, "On the design of RF spiral inductors on silicon," IEEE Transactions on Electron Devices, vol. 50, no. 3, pp. 718-729, Mar. 2003.

[42] M. Danesh, "Differentially driven symmetric microstrip inductors," IEEE Transactions on Microwave Theory and Techniques, vol. 50, no. 1, pp. 332-341, Jan. 2002.

[43] P. Andreani, "On the use of MOS varactors in RF VCO's," IEEE Journal of SolidState circuits, vol. 35, no. 6, pp. 905-910, June 2000.

[44] S. Mohan, "Modeling and characterization of on-chip transformers," in IEDM's, Dec. 1998.

[45] C. Yue, "On-chip spiral inductors with patterned ground shields for Si-based RF IC's," IEEE Journal of Solid-State Circuits, vol. 33, no. 5, pp. 743-752, Jun. 1997.

[46] A. Bonfanti, "Flicker Noise Up-Conversion due to harmonic distortion in Van der Pol CMOS oscillators," IEEE Transactions on circuits and systems, vol. 59, no. 7, pp. 1418-1430, Jul. 2012.

[47] H. Guo, "Closed-loop gain analysis of LC Quadrature VCO for the accurate prediction of oscillation frequency," in $M N R C$, Oct. 2009.

[48] J. Lopez-Villegas, "Improvement of the quality factor of RF integrated inductors by layout optimization," IEEE Transaction on Microwave Theory and Techniques, vol. 
48, no. 1, pp. 76-83, Jan. 2000.

[49] R. Middlebrook, "The general feedback therom: a final solution for feedback system," IEEE Microwave Magazine, vol. 7, no. 2, April 2006.

[50] R. Rao, "Statistical analysis of subthreshold leakage current for VLSI circuits," IEEE Transactions on Very Large Scale Intergration (VLSI) Systems, vol. 12, no. 2, pp. 131-139, Feb. 2004. 


\section{Appendices}

\section{Appendix A Middlebrook Method}

Middlebrook's method provides a way of finding accurate closed-loop gains in a feedback system. In conventional feedback system analysis, the loop gain is obtained in the following manner: first, the loop is broken to reach the feedback ratio, $K$, hence the loop gain $T$ can be determined by

$$
T=A K
$$

where $A$ is the forward gain. Then, the closed-loop gain $H$ is given by

$$
H=\frac{A K}{1+A K}
$$

However, in the actual hardware circuit, this conventional method of analysis is inaccurate. For instance, the input impedance of an op-amp is not infinite, and thus the signal transmission is bidirectional. Also, the DC operating points and small signal AC impedance on both sides of the breaking point are normally different.

Middlebrook's method can solve the problems mentioned above. By injecting a small test voltage signal $V_{t}$ and a small current signal $I_{t}$ Figure A.2, the voltage gain $T_{v}$ and current gain $T_{i}$ can be given respectively by

$$
\begin{gathered}
T_{v}=\frac{V_{f}}{V_{t}} \\
T_{i}=\frac{I_{f}}{I_{t}} \\
T=\frac{T_{v} \cdot T_{i}-1}{T_{v}+T_{i}+2} .
\end{gathered}
$$




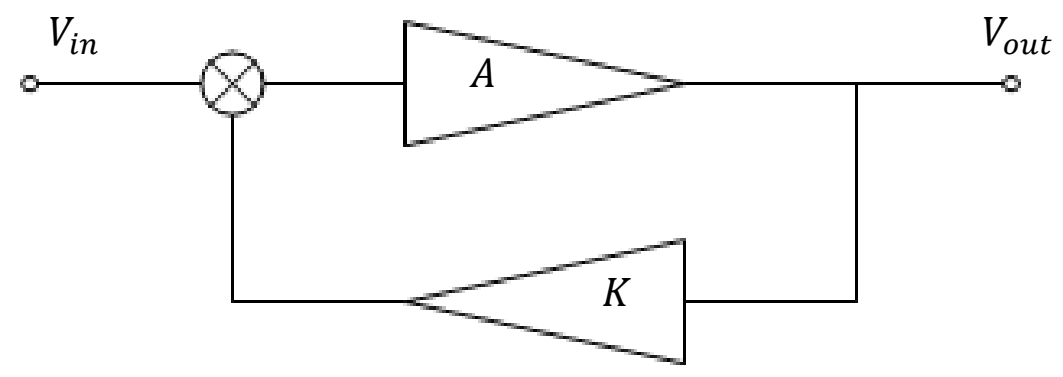

Figure A.1: Block diagram for typical negative feedback system

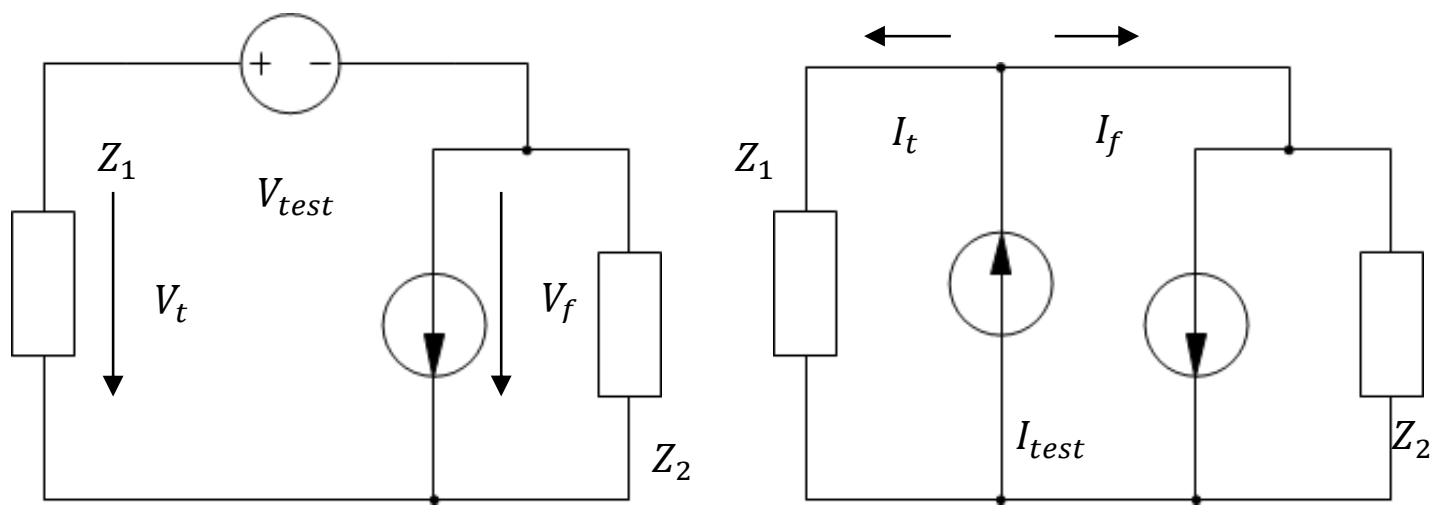

Figure A.2: Testing signal placement. (a) Voltage (b) Current

Note that the both $T_{v}$ and $T_{i}$ are complex gain. 


\section{Appendix B Definition of Q}

There are many definitions for the quality factor $\mathrm{Q}$ in various applications.

\section{B.1 Energy Approach}

The one most widely used definition is from the energy approach, which is the energy stored, divided by the energy dissipated per cycle:

$$
Q_{E}=\omega \frac{E_{\text {stored }}}{E_{\text {diss }}}
$$

This definition is very general and can be applied in multiple contexts, from optical systems to mechanical systems. Providing an analogy of this definition, Q of passive components (inductors and capacitors) can be defined as,

$$
Q_{c, L}=\frac{\text { reactive impedance }}{\text { real impedance }}=\frac{i m(y 11)}{\text { re }(y 11)}
$$

\section{B.2 Bandwidth Approach}

From the bandwidth approach, Q is defined for bandpass characteristics as the center frequency divided by the $3 \mathrm{~dB}$ bandwidth is

$$
Q_{B W}=\frac{\omega_{c}}{\Delta \omega_{3 d B}}
$$

\section{B.3 Stability Approach}


From the phase stability approach, $Q$ is defined as the slope of the phase at center frequency, given that

$$
Q_{\text {phase }}=-\frac{\omega_{c}}{2} \frac{d \varphi}{d \omega} \mid \omega=\omega_{c} .
$$

However, no matter the approach, when applying these definitions to an LC tank, Q may be derived into a similar expression, or

$$
Q=\omega \frac{L_{s}}{R_{S}}
$$

where $L_{S}$ and $R_{S}$ represent the series inductance and series resistance, respectively. 


\section{Appendix C Simulation Issues on Inductors}

Since transformers have been employed in this topology, using the existing inductor models in the library provided by the foundry has become impossible. Instead, the inductor should be modeled by an inductor connected with a resistor in series. The aim of adding the resistor is to model the parasitic resistance of the inductor.

To get the inductance and parasitic resistance at the oscillating frequency, a port is connected to the inductors in parallel and ground one side to implement characterization. Then the quality factor, inductance and parasitic resistance can be obtained through simulations by inductors

$$
\begin{aligned}
& Q=\frac{\operatorname{Im}(Z 11)}{\operatorname{Re}(Z 11)} \\
& L=\frac{\operatorname{Im}(Z 11)}{\omega} \\
& R=\operatorname{Re}(Z 11) .
\end{aligned}
$$

Then these values can be used in the VCO simulation. 


\section{Appendix D Issues on Transformers}

The transformers can be modeled using mind elements in analoglib library in Cadence. However, the problem is how to estimate the coupling coefficients $k$ for on chip transformers. Although many software can be used to get accurate $k$, it is unworthy to consume so much time using those software to guide VCO designing. In fact, for tapped and interleaved transformers, the inductances can be calculated from geometric parameters, such as the outer dimension, coil width spacing and number of turns. Therefore, an easy approximation in [44] is used here to find an estimation of $k$.

First, mutual inductance can be found by

$$
M=\left(L_{T}-L_{1}-L_{2}\right) / 2
$$

where $L_{T}$ is the total inductance of a single spiral with all the segments of both primary and secondary traces, $L_{1}$ and $L_{2}$ are the self-inductances of the primary and secondary coil respectively, and $M$ is the mutual inductance.

Then $k$ can be calculated by

$$
k=\frac{M}{\sqrt{L_{1} L_{2}}}
$$




\section{Appendix E Tank Inductor}

Except for the turns $(N)$ of differential inductors, another three parameters that determine the performance of inductors are: outer dimension $(O)$, coil width $(W)$ and spacing $(S)$ (see Fig. Figure E.1). Quality factor $(Q)$, inductance $(L)$ and parasitic resistance $(R)$ can be calculated with these parameters, however usually it is not accurate. Hence simulations are taken to help choosing the appropriate parameters for the inductors. High quality factor and high inductance are desirable.

Fig. Figure E. 2 - Fig.Figure E. 4 show how $N$ influence $Q, L$ and $R$ with certain $O$, $W$ and $S$ when $N=1,2,3$. Table 21 concludes the results. It turns out $N$ is a crucial factor to determine $Q, L$ and $R$. Considering sparing area for the secondary coil, the maximum of the turns is set to be 3 . When $N=3$, although $R$ is largest ( $R$ is proportional to the length of the inductor), it still provides largest $Q$ and $L$ around the target center frequency (2.4GHz). Hence $N$ is chosen to be equal to 3 .

Fig. Figure E.5 - Fig. Figure E.7 demonstrate how $O$ influence $Q, L$ and $R$ with certain $W, S$ when $N=3$. Results are concluded in Table 22. Around $2.4 \mathrm{GHz}, Q, L$ and $R$ increase with the increment of $O$. Hence, to obtain largest $Q$ and $L$, as well as providing more area to the secondary coil, $O$ is chosen at $300 \mathrm{um}$.

Fig Figure E. 8 - Fig. Figure E. 10 show how $W$ influence $Q, L$ and $R$ with certain $S$ when $N=3$ and $O=300 \mathrm{um}$. Results are concluded in Table 23. It turns out larger width is harmful to both quality factor and inductance. Hence, $W$ is set as the minimal width that provides by the technology.

Fig. Figure E.11 - Fig. Figure E.13 demonstrate how $S$ influence $Q, L$ and $R$ with when $N=3, O=300 \mathrm{um}$ and $W=8.5 \mathrm{um}$. Table 24 concludes the results. It turns out larger $\mathrm{S}$ is harmful to both quality factor and inductance, but does not affect $R$ severely. Taking saving area for secondary coil into account, $S$ is set to the minimal in this technology. 


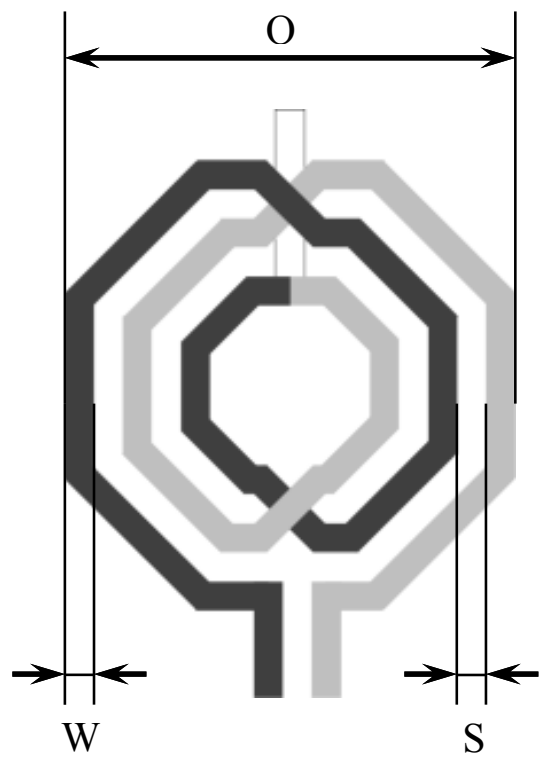

Figure E.1: Parameters of inductors.

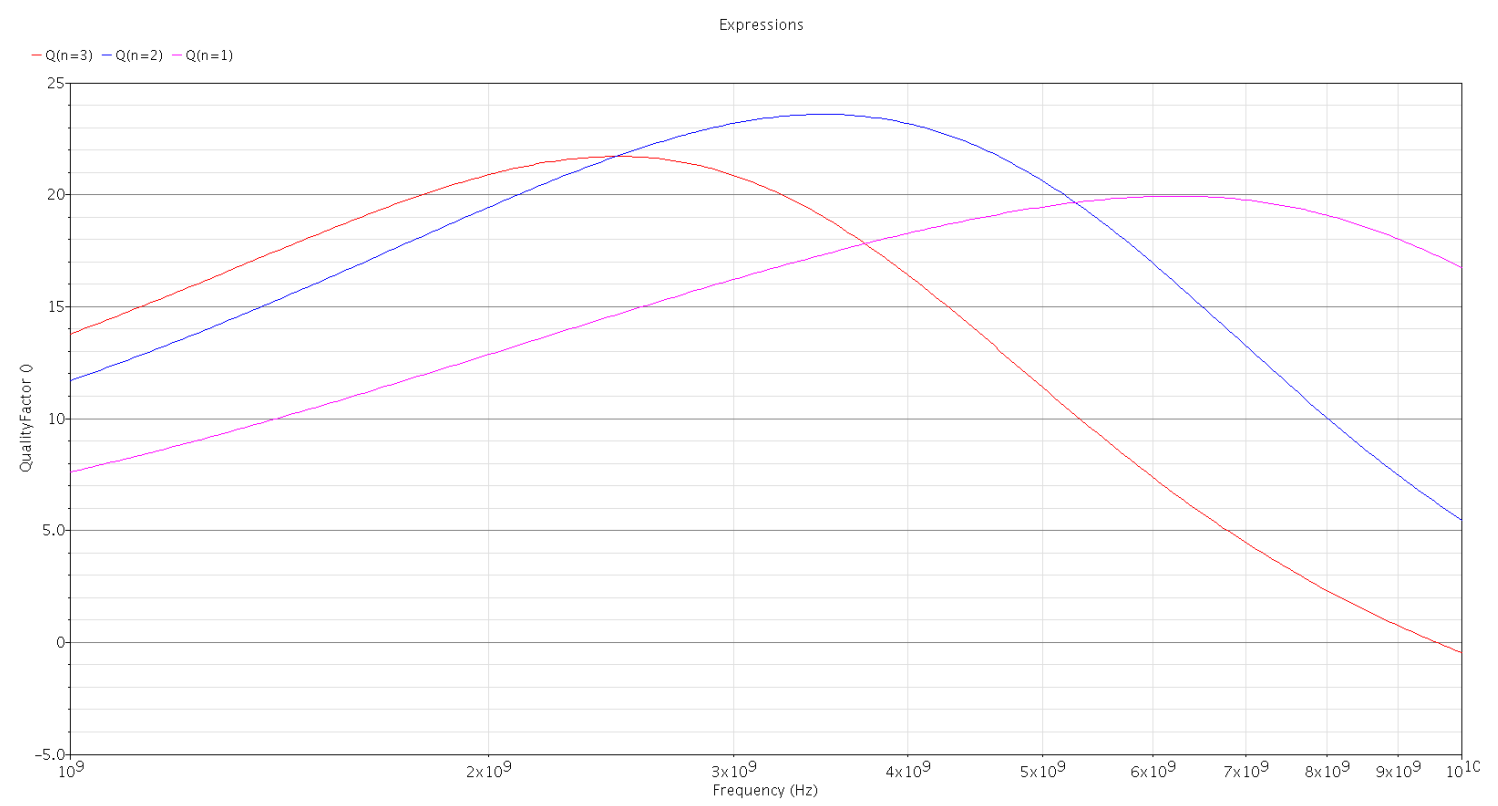

Figure E.2: Quality factor vs. Turns. 


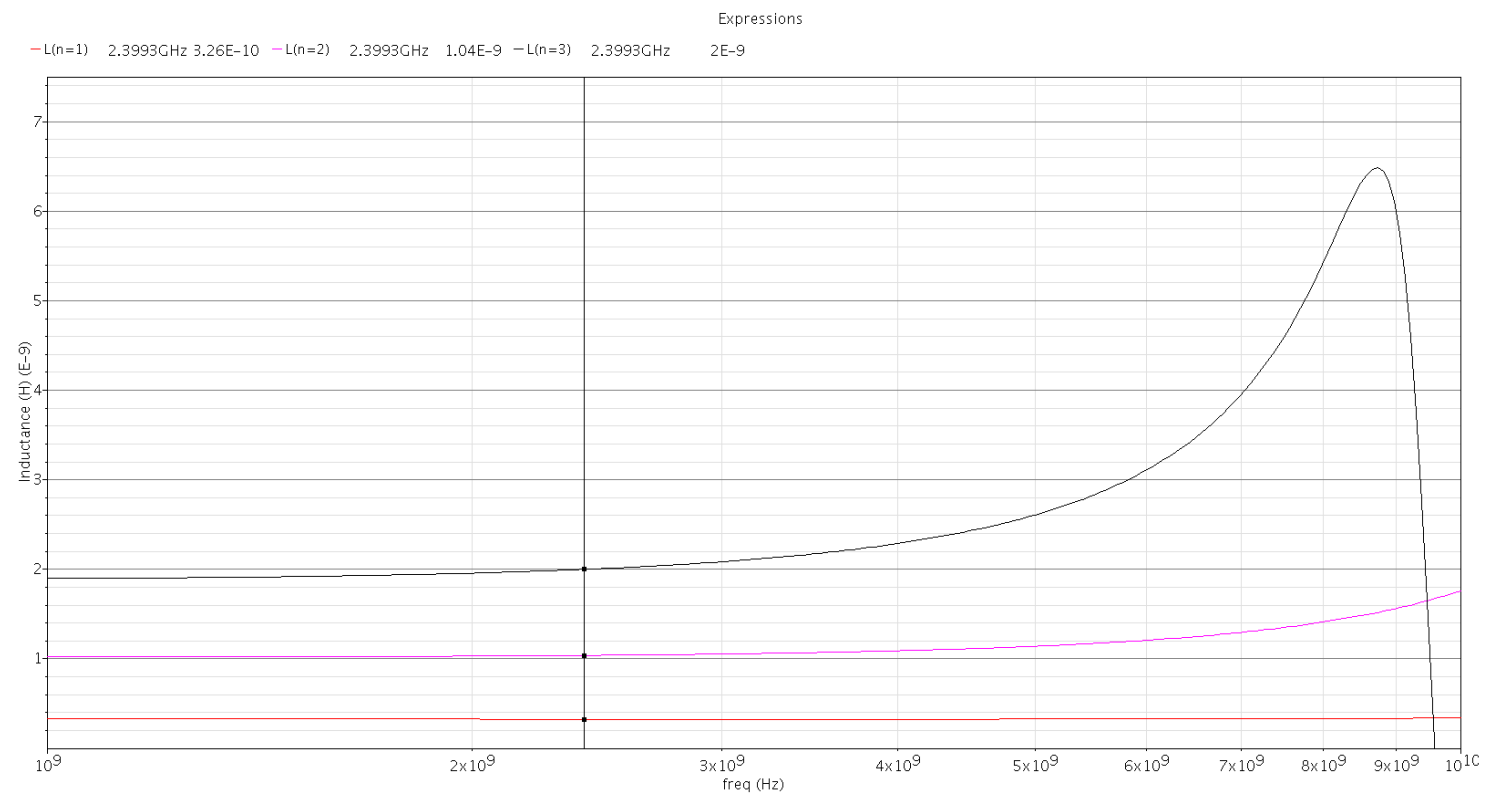

Figure E.3: Inductance vs. Turns.

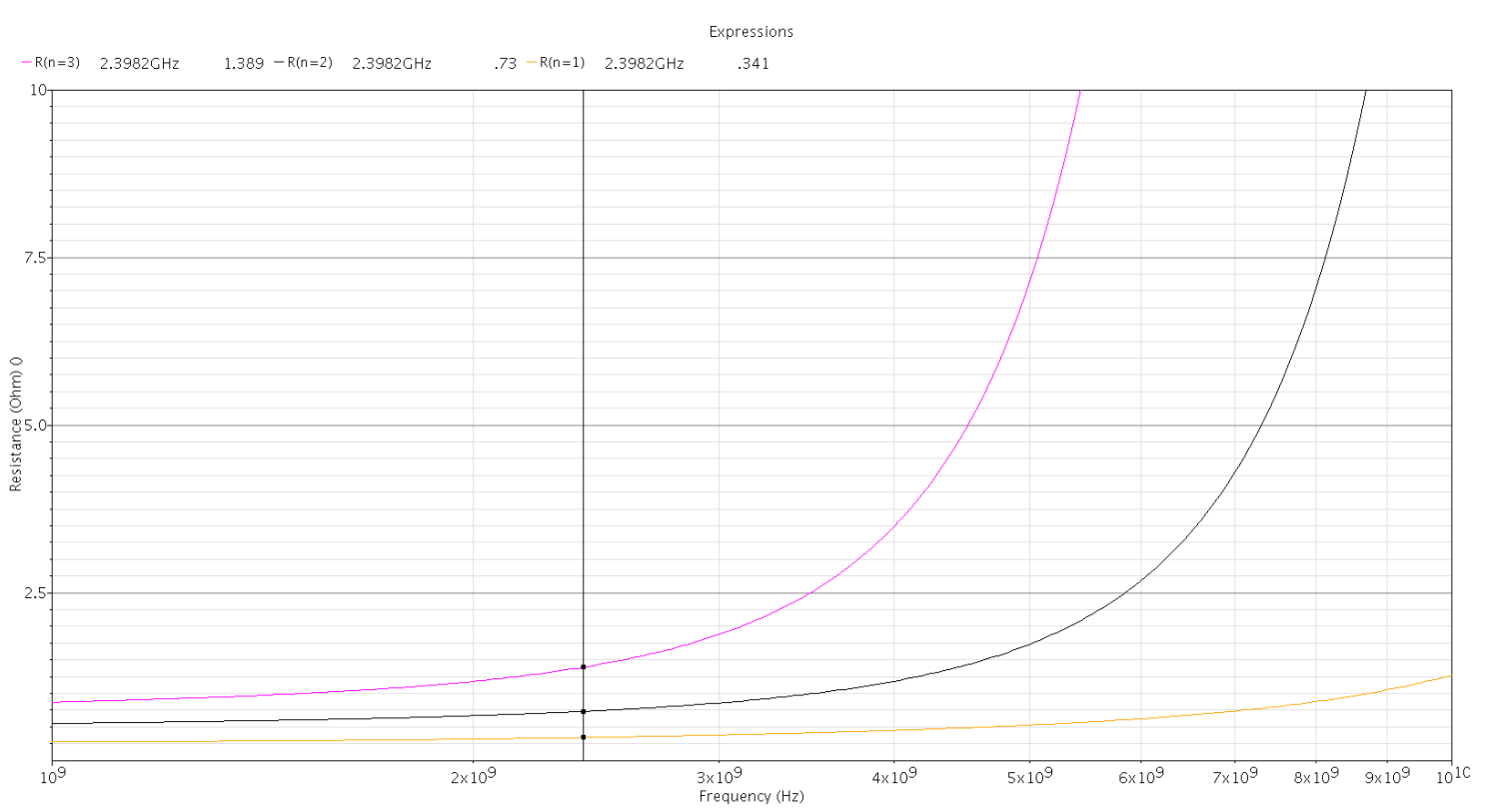

Figure E.4: Resistance vs. Turns. 
Expressions 1

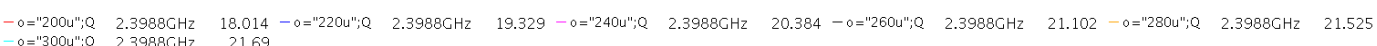

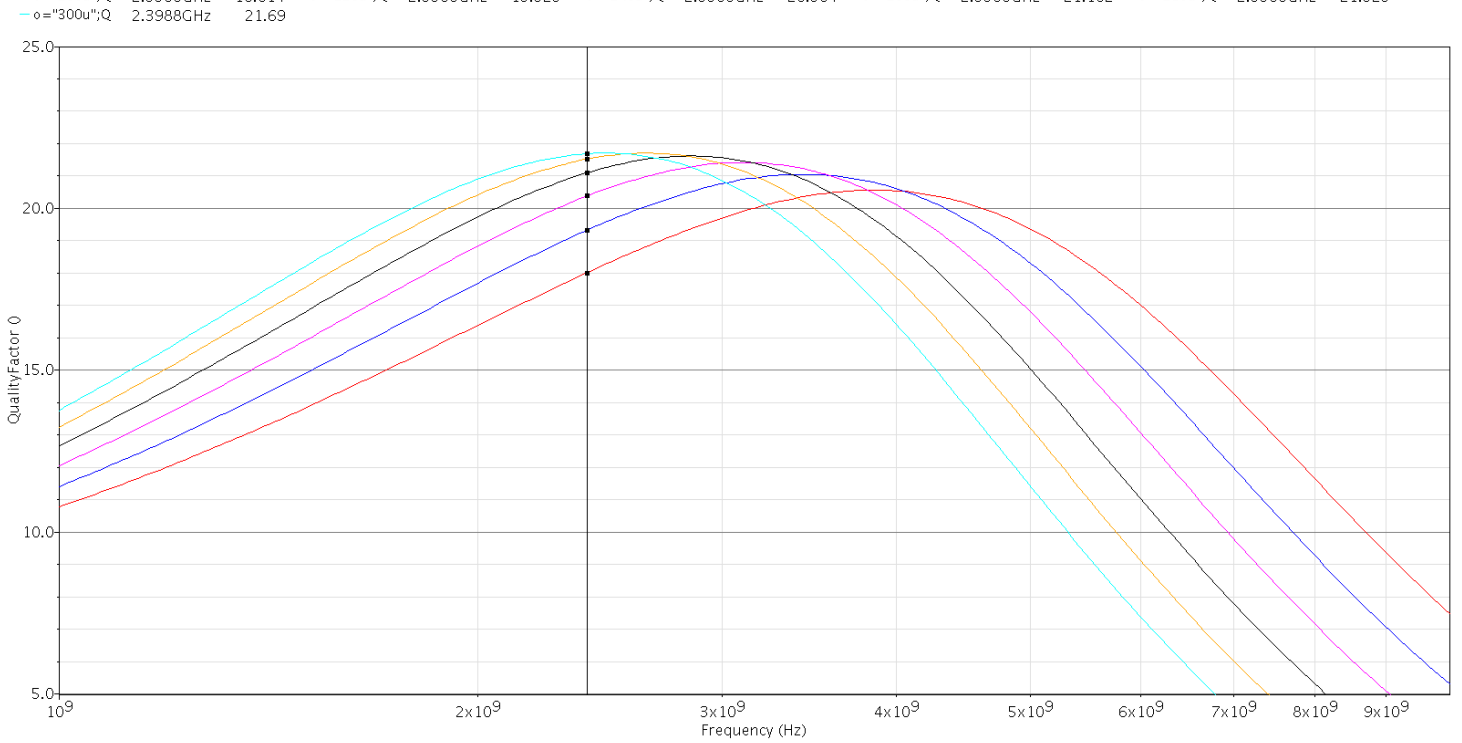

Figure E.5: Quality factor vs. Outer dimension.

Expressions 2

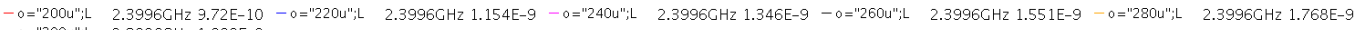
3996GHz 1.999E-9

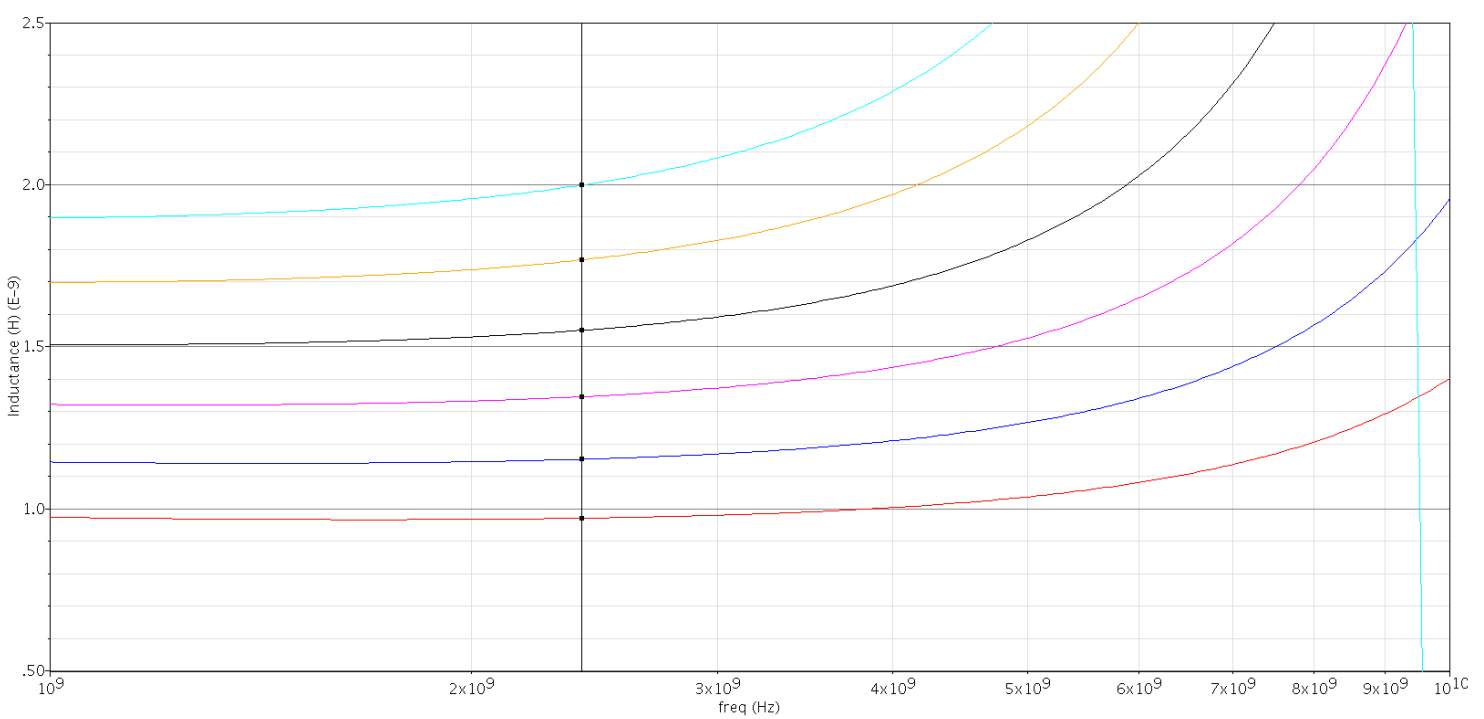

Figure E.6: Inductance vs. Outer dimension 


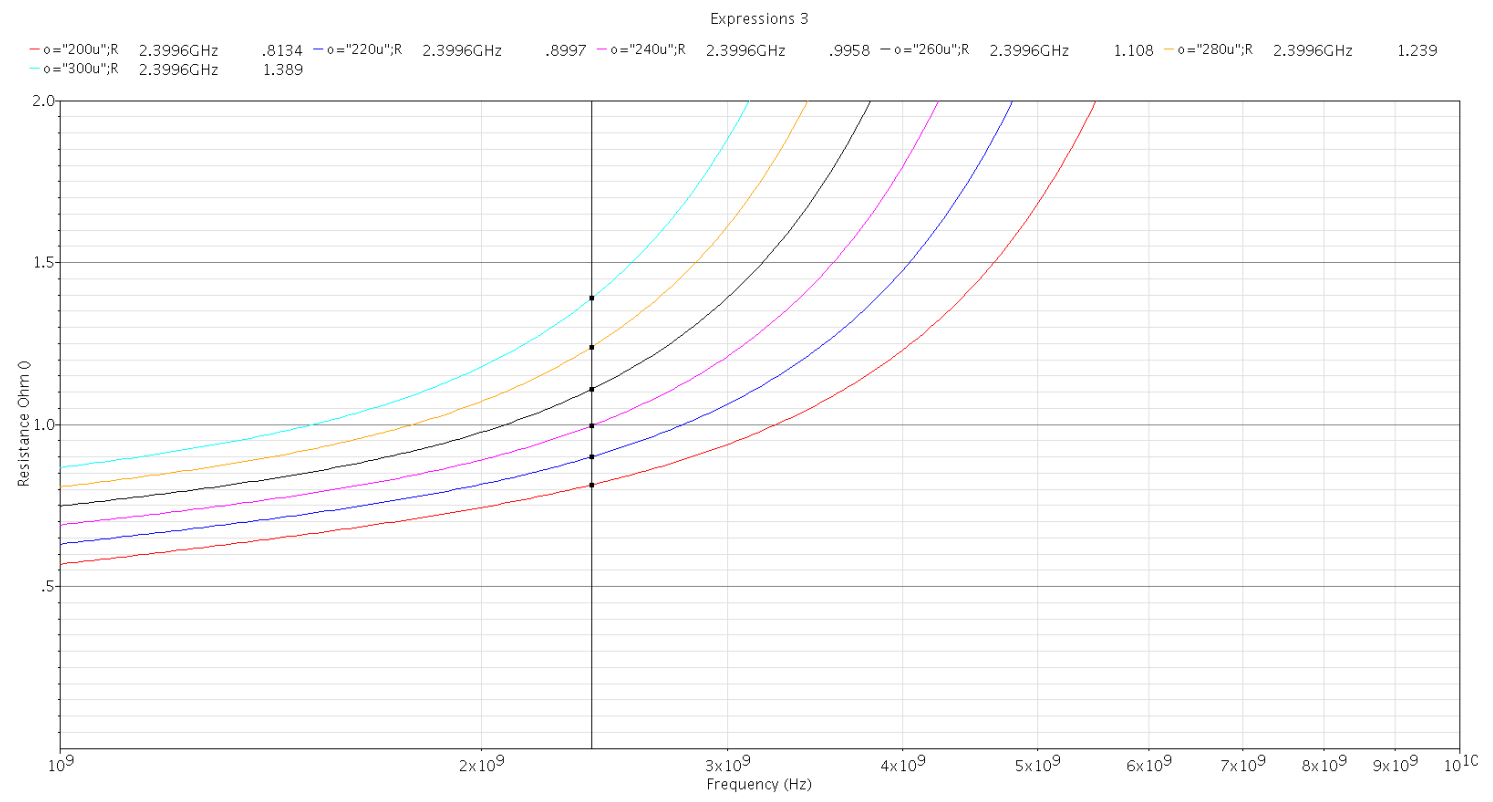

Figure E.7: Resistance vs. Outer dimension.

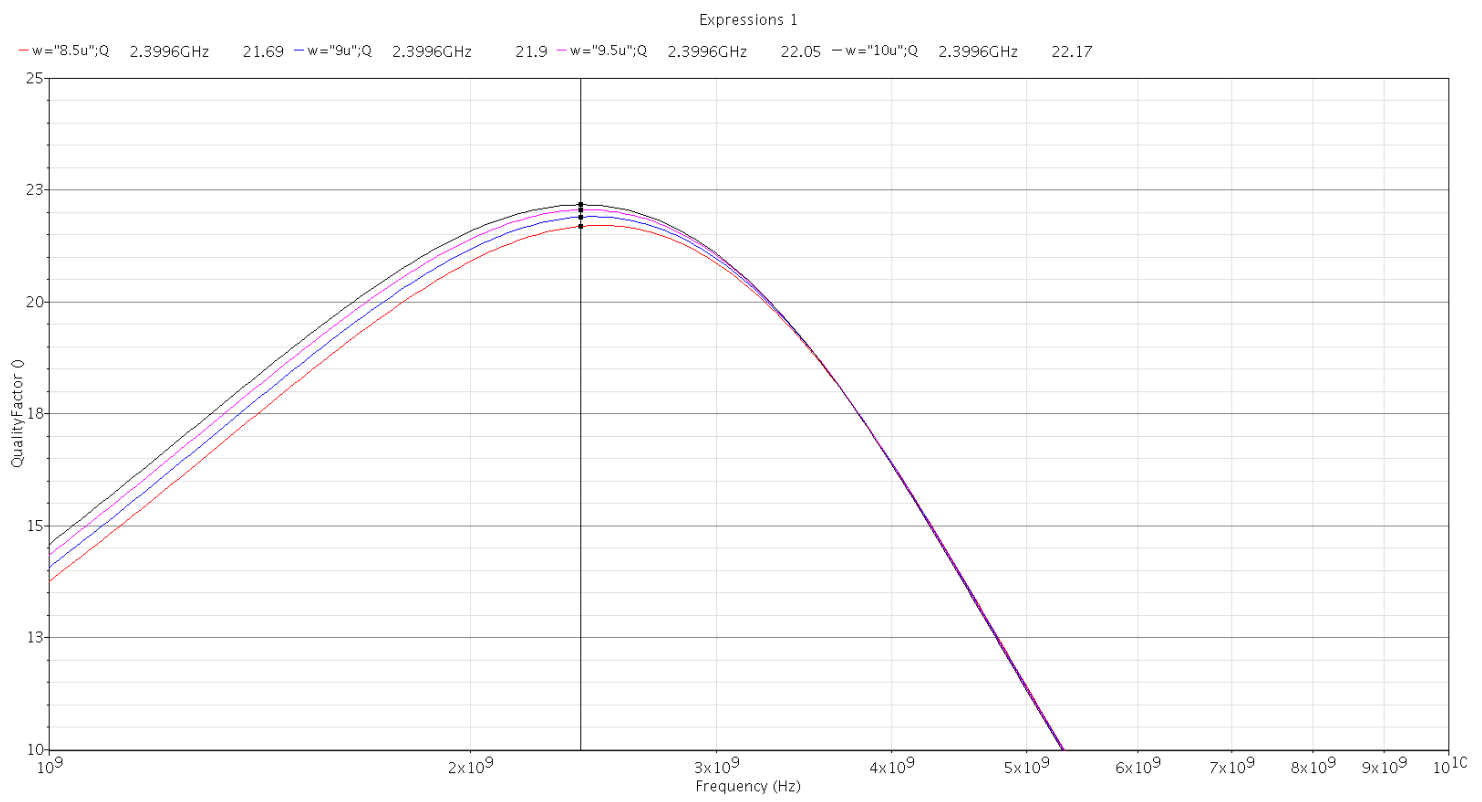

Figure E.8: Quality factor vs. Coil width 
Expressions

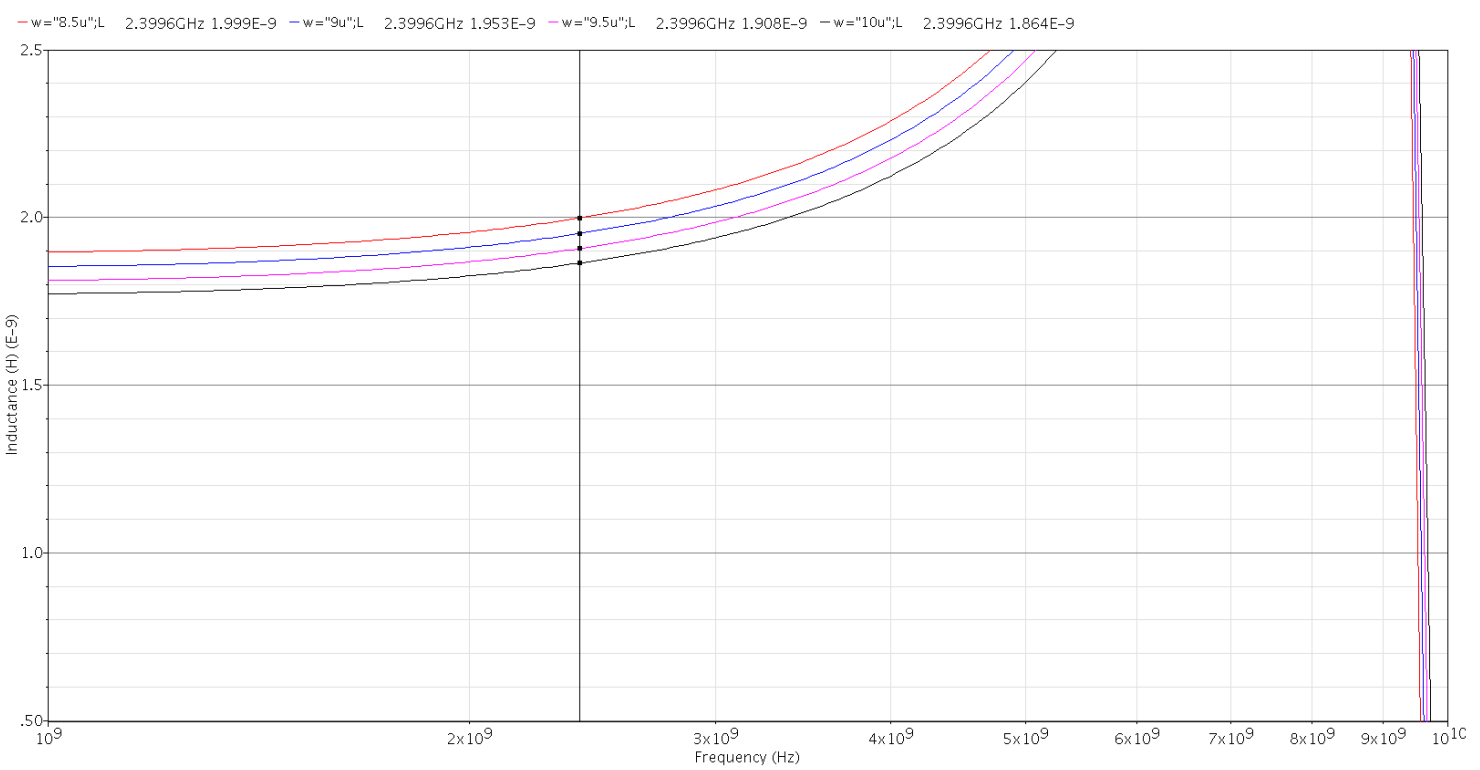

Figure E.9: Inductance vs. Coil width.

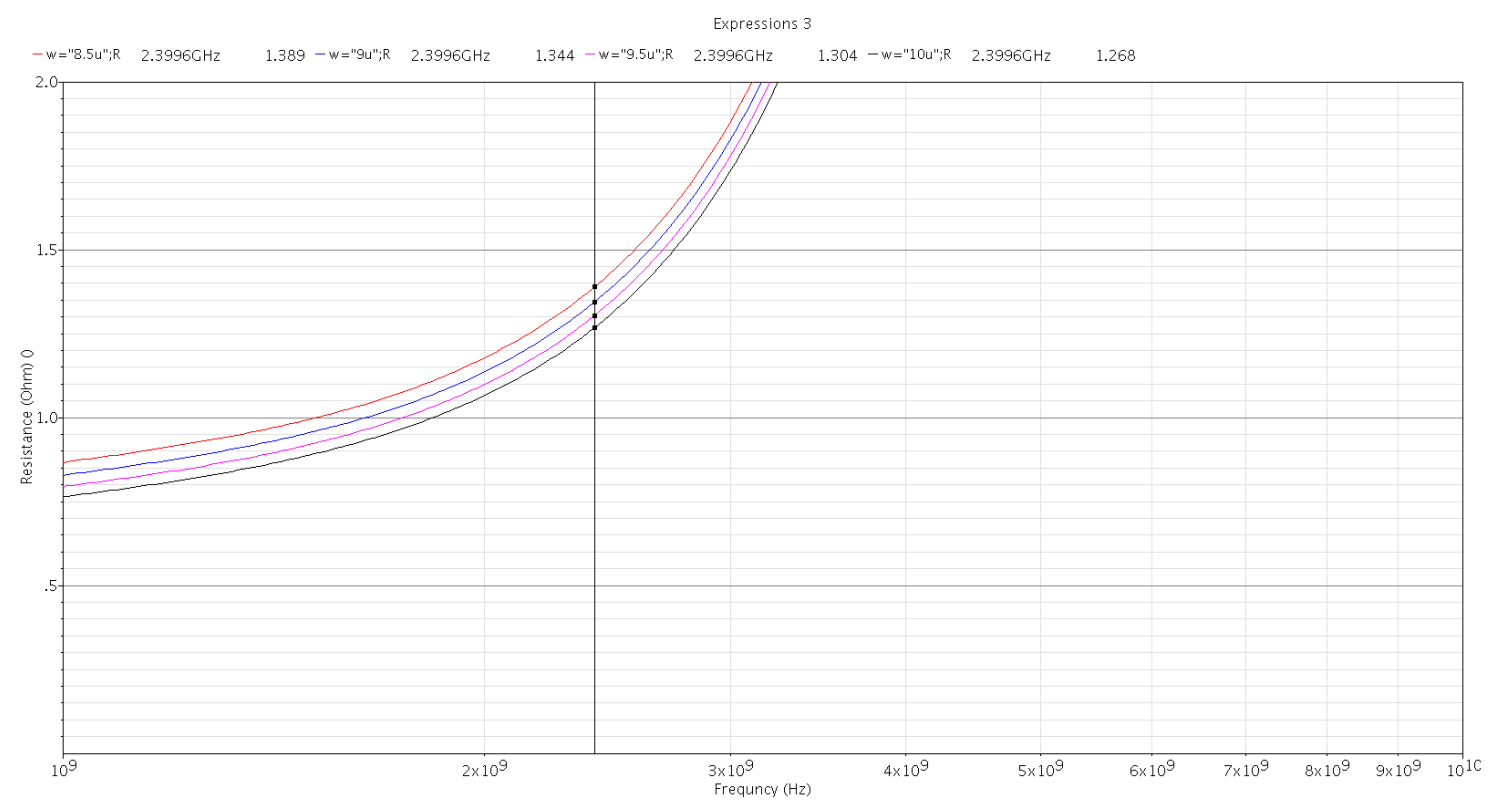

Figure E.10: Resistance vs. Coil width. 
Expressions 1

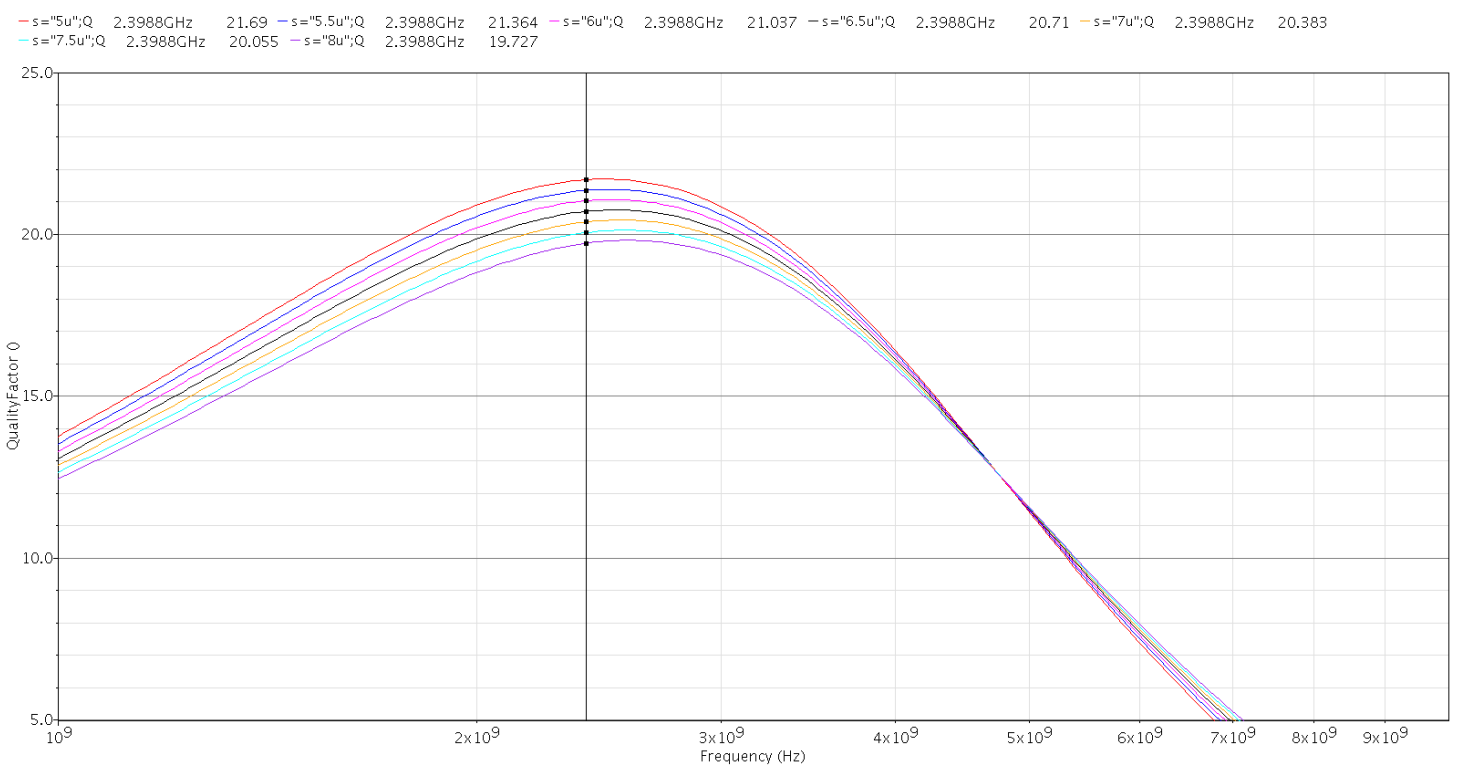

Figure E.11: Quality factor vs. Space

Expressions 2

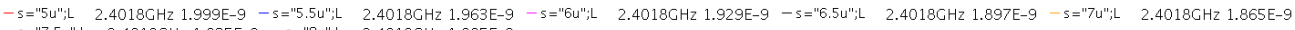

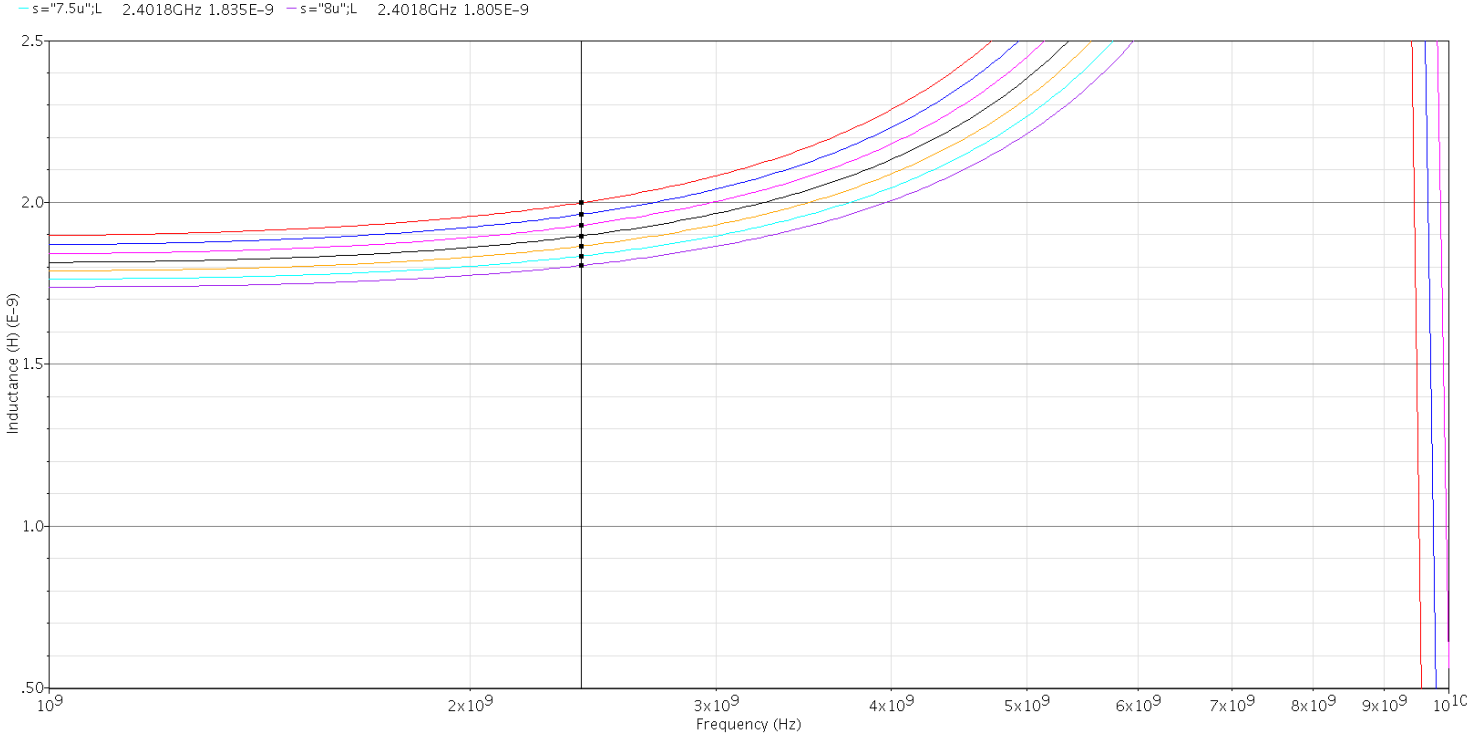

Figure E.12: Inductance vs. Space 


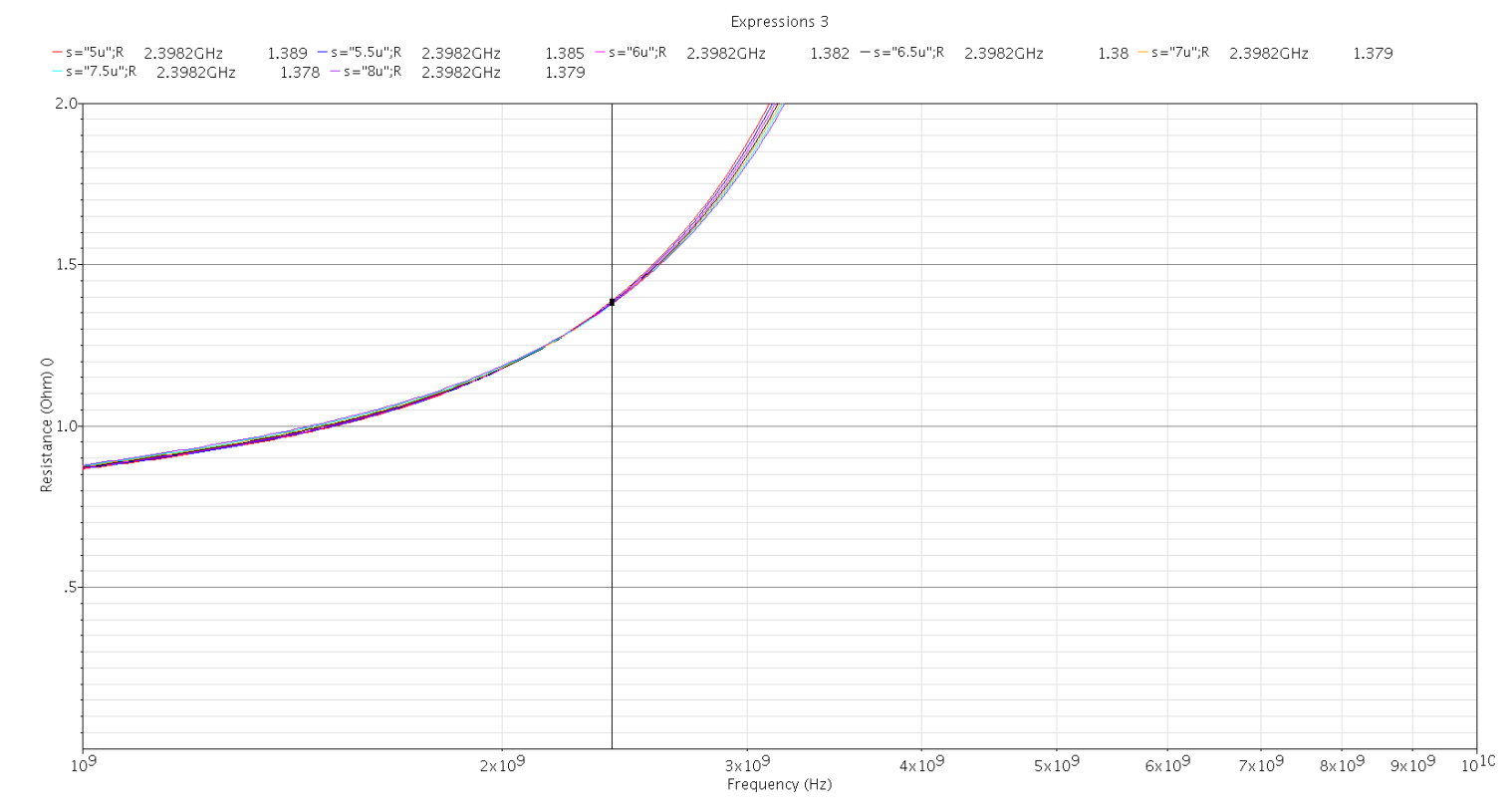

Figure E.13: Resistance vs. Space

Table 21: $Q, L, R$ vs. $N$ at $2.4 \mathrm{GHz}$.

\begin{tabular}{c|c|c|c}
\hline & $\boldsymbol{Q}$ & $\boldsymbol{L} \mathbf{( n H )}$ & $\boldsymbol{R}(\mathbf{o h m})$ \\
\cline { 1 - 2 } $\boldsymbol{N} \boldsymbol{N}=\mathbf{1}$ & 15.4 & 0.126 & 0.342 \\
\cline { 1 - 1 } $\boldsymbol{N}=\mathbf{2}$ & 21.2 & 1.04 & 0.71 \\
\cline { 1 - 1 } $\boldsymbol{N}=\mathbf{3}$ & 21.7 & 2.00 & 1.389 \\
\hline
\end{tabular}

Table 22: $Q, L, R$ vs. $O$ when $N=3$ at $2.4 \mathrm{GHz}$.

\begin{tabular}{c|c|c|c}
\hline \multirow{2}{*}{$\boldsymbol{O}=\mathbf{2 0 0 u m}$} & $\boldsymbol{Q}$ & $\boldsymbol{L} \mathbf{( n H )}$ & $\boldsymbol{R}(\mathbf{o h m})$ \\
\cline { 1 - 1 } $\boldsymbol{O} \boldsymbol{O}=\mathbf{2 2 0 u m}$ & 18.014 & 0.972 & 0.8134 \\
\cline { 1 - 1 } $\boldsymbol{O}=\mathbf{2 4 0 u m}$ & 19.329 & 1.154 & 0.8997 \\
\cline { 1 - 1 } $\boldsymbol{O}=\mathbf{2 6 0 u m}$ & 20.384 & 1.346 & 0.9958 \\
\cline { 1 - 1 } $\boldsymbol{O}=\mathbf{2 8 0 u m}$ & 21.102 & 1.551 & 1.108 \\
\cline { 1 - 1 } $\boldsymbol{O}$ & 21.525 & 1.768 & 1.239 \\
\hline
\end{tabular}


Table 23: $Q, L, R$ vs. $W$ when $N=3$ and $O=300 \mathrm{um}$ at $2.4 \mathrm{GHz}$.

\begin{tabular}{c|c|c|c}
\hline & $\boldsymbol{Q}$ & $\boldsymbol{L}(\mathbf{n H})$ & $\boldsymbol{R}(\mathbf{o h m})$ \\
\cline { 1 - 2 } $\boldsymbol{y} \boldsymbol{W}=\mathbf{8 . 5 u m}$ & 21.69 & 2.00 & 1.389 \\
\cline { 1 - 1 } $\boldsymbol{W} \boldsymbol{W}=\mathbf{9 . 0 u m}$ & 21.9 & 1.953 & 1.344 \\
\cline { 1 - 1 } $\boldsymbol{y} \boldsymbol{W}=\mathbf{1 0 . 0 u m}$ & 22.05 & 1.908 & 1.304 \\
& 22.17 & 1.864 & 1.268 \\
\hline
\end{tabular}

Table 24: $Q, L, R$ vs. $S$ when $N=3, O=300 \mathrm{um}$ and $W=8.5 \mathrm{um}$ at $2.4 \mathrm{GHz}$

\begin{tabular}{|c|c|c|c|}
\hline & $Q$ & $L(\mathrm{nH})$ & $R(\mathrm{ohm})$ \\
\hline$S=5.0 \mathrm{um}$ & 21.69 & 2.00 & 1.389 \\
\hline$S=5.5 \mathrm{um}$ & 21.364 & 1.963 & 1.385 \\
\hline$S=6.0 \mathrm{um}$ & 21.037 & 1.929 & 1.382 \\
\hline$S=6.5 \mathrm{um}$ & 20.71 & 1.897 & 1.38 \\
\hline$S=7.0 \mathrm{um}$ & 20.383 & 1.865 & 1.379 \\
\hline$S=7.5 \mathrm{um}$ & 20.055 & 1.835 & 1.378 \\
\hline$S=8.0 u m$ & 19.727 & 1.805 & 1.379 \\
\hline
\end{tabular}

Note that all the inductance and resistance are single-ended value. 